\title{
COOL BOTTOM PROCESSES ON THE THERMALLY PULSING ASYMPTOTIC GIANT BRANCH AND THE ISOTOPIC COMPOSITION OF CIRCUMSTELLAR DUST GRAINS
}

\author{
Kenneth M. Nollett \\ MC 130-33, California Institute of Technology, Pasadena, CA 91125;nollett@phys.washington.edu \\ M. Busso \\ Department of Physics, University of Perugia, Via Pascoli, 06100 Perugia, Italy; busso@fisica.unipg.it \\ AND \\ G. J. WASSERBURG \\ The Lunatic Asylum, Division of Geological and Planetary Sciences, California Institute of Technology, Pasadena, CA 91125; \\ isotopes@gps.caltech.edu \\ Received 2002 January 21; accepted 2002 September 20
}

\begin{abstract}
We examine the effects of cool bottom processing $(\mathrm{CBP})$ on the isotopic ratios ${ }^{18} \mathrm{O} /{ }^{16} \mathrm{O},{ }^{17} \mathrm{O} /{ }^{16} \mathrm{O},{ }^{14} \mathrm{~N} /{ }^{15} \mathrm{~N}$, ${ }^{26} \mathrm{Al} /{ }^{27} \mathrm{Al}, \mathrm{C} / \mathrm{O}$, and $\mathrm{N} / \mathrm{O}$ in the convective envelope during the thermally pulsing asymptotic giant branch (TP-AGB) phase of evolution in a $1.5 M_{\odot}$ initial mass star of solar initial composition. We use a parametric model that treats extra mixing by introducing mass flow between the convective envelope and the underlying radiative zone. The parameters of this model are the mass circulation rate $(\dot{M})$ and the maximum temperature $\left(T_{P}\right)$ experienced by the circulating material. The effects of nuclear reactions in the flowing matter were calculated using a set of static structures of the radiative zone selected from particular times in a complete stellar evolution calculation. The compositions of the flowing material were obtained, and the resulting changes in the envelope determined. No major shifts in the star's energy budget occur from the imposed CBP if $\log T_{P}<7.73$. Using structures from several times on the TP-AGB, it was found that the results for all species except ${ }^{26} \mathrm{Al}$ were essentially independent of the time chosen if $\log T_{P}>7.6$. Abundant ${ }^{26} \mathrm{Al}$ was produced by CBP for $\log T_{P}>7.65$. While ${ }^{26} \mathrm{Al} /{ }^{27} \mathrm{Al}$ depends on $T_{P}$, the other isotopic ratios depend dominantly on the circulation rate. The relationship is shown between models of CBP as parameterized by a diffusion formalism within the stellar evolution model and those using the mass-flow formalism employed here. They are shown to be effectively equivalent. In general, the CBP treatment readily permits calculation of envelope compositions as affected by different degrees of extra mixing, based on stellar structures computed by normal stellar evolution models. Using these results, the isotopic ratios under conditions of $\mathrm{C} / \mathrm{O}<1$ and $\mathrm{C} / \mathrm{O}>1$ are compared with the data on circumstellar dust grains. It is found that the ${ }^{18} \mathrm{O} /{ }^{16} \mathrm{O},{ }^{17} \mathrm{O} /{ }^{16} \mathrm{O}$, and ${ }^{26} \mathrm{Al} /{ }^{27} \mathrm{Al}$ observed for oxide grains formed at $\mathrm{C} / \mathrm{O}<1$ are reasonably well understood. However, the ${ }^{15} \mathrm{~N} /{ }^{14} \mathrm{~N}$, ${ }^{12} \mathrm{C} /{ }^{13} \mathrm{C}$, and ${ }^{26} \mathrm{Al} /{ }^{27} \mathrm{Al}$ in carbide grains $(\mathrm{C} / \mathrm{O}>1)$ require that many of their stellar sources must have had ${ }^{14} \mathrm{~N} /{ }^{15} \mathrm{~N}$ at least a factor of 4 lower than the solar value. This allows a self-consistent description of all these isotopes in most $\mathrm{SiC}$ grains. The rare grains with ${ }^{12} \mathrm{C} /{ }^{13} \mathrm{C}<10$ cannot be produced by any red giant or AGB source, nor are they reconcilable with novae sources.
\end{abstract}

Subject headings: Galaxy: abundances — meteors, meteoroids nuclear reactions, nucleosynthesis, abundances — solar system: formation stars: abundances — stars: AGB and post-AGB

\section{INTRODUCTION}

The purpose of this study is to explore the effects of an extra circulation process on the ${ }^{26} \mathrm{Al} /{ }^{27} \mathrm{Al},{ }^{17} \mathrm{O} /{ }^{16} \mathrm{O}$, ${ }^{18} \mathrm{O} /{ }^{16} \mathrm{O}$, and ${ }^{13} \mathrm{C} /{ }^{12} \mathrm{C}$ ratios in the envelope of an asymptotic giant branch (AGB) star of solar metallicity. By " an extra circulation process," we mean the transport of matter from the fully convective envelope into the underlying radiative region, down to the outer parts of the H-burning shell. The actual physical-dynamical basis for such penetration is not known. However, the requirement that some such mechanism must sometime occur has been amply demonstrated by observations on red giant branch (RGB) stars and in studies of dust grains that formed in circumstellar envelopes (e.g., Huss et al. 1994; Boothroyd, Sackmann, \& Wasserburg 1994; Harris, Lambert, \& Smith 1985; Kahane et al. 1992). The isotopic effects of such extra transport on the abundances depend only on the degree of nuclear pro- cessing. This is directly related to the nuclear reaction rates, the rate of mass transfer, and the depth to which the extramixing mechanism penetrates. A detailed physical fluiddynamical mechanism cannot significantly affect the resulting abundances beyond determining the rate and depth. It is clear that any extra-mixing mechanism that does not fractionate one set of elements relative to the other elements involved in the nuclear reactions will not change the resulting products that are added to the envelope from what is found here. The issue of how small amounts of extra mixing will change the stellar evolution is not known but will be commented on. The results, presented here with interrelationships between the different isotopic ratios, are of use in discussing and understanding isotopic results obtained by extensive studies of recovered circumstellar dust grains. Insofar as a relationship may be found between the isotopic ratios listed above and the ratios of major elements in the AGB envelope $(\mathrm{C} / \mathrm{O}$ and $\mathrm{N} / \mathrm{O})$, this may lead to under- 
standing the chemical-physical origins of the grains and the nature of the stars around which they formed. Such results may also be of value in understanding the compositions of planetary nebulae.

It has been proposed that many short-lived nuclei present in the early solar system were the products of an AGB star that injected freshly synthesized nuclei into the interstellar medium (ISM) from which the solar system formed (Wasserburg et al. 1994). Because AGB stars are the site of $s$-process nucleosynthesis, there is a rather thorough understanding of the yields of a large number of nuclides in their convective envelopes (see Busso, Gallino, \& Wasserburg 1999, hereafter BGW99). Among these nuclides, ${ }^{26} \mathrm{Al}$ plays a critical role in evaluating the hypothesis that an AGB star supplied short-lived nuclei to the early solar system. While it is clear that ${ }^{26} \mathrm{Al}$ should be produced in AGB stars by the reaction ${ }^{25} \mathrm{Mg}(p, \gamma){ }^{26} \mathrm{Al}$ (Forestini, Arnould, \& Paulus 1991; Mowlavi \& Meynet 2000; Lattanzio, Forestini, \& Charbonnel 2000), the quantitative ${ }^{26} \mathrm{Al}$ yield, taking into account the complexities of destruction of this nuclide, remains very uncertain. As shown by Wasserburg et al. (1994) and BGW99, ${ }^{26} \mathrm{Al}$ is produced efficiently in the hydrogen shell, but most $(\sim 90 \%)$ of this is mixed into the He shell at the onset of intermittent He-shell burning. The mixed material is then exposed to neutrons in the convective (He-burning) region formed during the He-burning pulse, where $80 \%-100 \%$ of its ${ }^{26} \mathrm{Al}$ content is consumed by the reaction ${ }^{26} \mathrm{Al}(n, p){ }^{26} \mathrm{Mg}$. The neutrons that destroy ${ }^{26} \mathrm{Al}$ are provided by the process ${ }^{22} \mathrm{Ne}(\alpha, n){ }^{25} \mathrm{Mg}$, activated marginally in the relatively high temperatures $\left(T \geq 3 \times 10^{8} \mathrm{~K}\right)$ of the thermal pulse. A value of ${ }^{26} \mathrm{Al} /{ }^{27} \mathrm{Al} \sim 10^{-3}$ (三number ratio) results in the envelope after mixing and is much less than the amount initially produced in the hydrogen shell. This is a factor of a few less than required if a single AGB star produced the ${ }^{26} \mathrm{Al}$ and this material was then diluted to provide the amount found in the early solar system ${ }^{26} \mathrm{Al} /{ }^{27} \mathrm{Al} \sim 5 \times 10^{-5}$; Lee, Papanastassiou, \& Wasserburg 1977; Wasserburg et al. 1994, 1995). Although the discrepancy might be explained by a relatively small change in the fraction of ${ }^{26} \mathrm{Al}$ destroyed [i.e., a small variation in the ${ }^{26} \mathrm{Al}(n, p){ }^{26} \mathrm{Mg}$ rate], the estimated ${ }^{26} \mathrm{Al}$ yield is at best in marginal agreement with the yield required to provide the solar inventory. If the true yield is lower than ${ }^{26} \mathrm{Al} /{ }^{27} \mathrm{Al} \sim 4 \times 10^{-3}$, then an $\mathrm{AGB}$ source for solar ${ }^{26} \mathrm{Al}$ is not reasonable. There is at present no way to improve the estimated ${ }^{26} \mathrm{Al}$ yield. Mowlavi \& Meynet (2000) recently carried out an extensive analysis of ${ }^{26} \mathrm{Al}$ production in $\mathrm{AGB}$ stars. They explored the effects of recently revised reaction rates and computed the yields expected at varying metallicities and initial masses. The results of this work agree with the above expectations: normal, low-mass AGB stars were found to be probable sources of ${ }^{26} \mathrm{Al}$ abundances observed in most (not all) $\mathrm{SiC}$ grains, with ${ }^{26} \mathrm{Al} /{ }^{27} \mathrm{Al}$ values in the same range discussed above. Mowlavi \& Meynet also found more efficient production of ${ }^{26} \mathrm{Al}$ in intermediate-mass stars experiencing hot bottom burning (HBB). This process was studied extensively by Frost et al. (1998), Lattanzio \& Forestini (1999), and Lattanzio et al. (2000). However, uncertainties in the final fates of these stars (mainly in the number of thermal pulses experienced) prevent a quantitative estimate of their possible contributions to the ISM and to the Galactic inventory.

A direct challenge to standard AGB models arises from the discovery of circumstellar oxide grains recovered from meteorites, on which rather precise isotopic ratios are measured. Many of these grains reflect ${ }^{26} \mathrm{Al} /{ }^{27} \mathrm{Al}$ at their formation far above $10^{-3}$. Some oxide grains also have ${ }^{18} \mathrm{O} /{ }^{16} \mathrm{O}$ about $30 \%$ less than the solar value and ${ }^{17} \mathrm{O} /{ }^{16} \mathrm{O}$ much greater than the solar value. These oxygen data were interpreted to reflect stellar first dredge-up (e.g., Huss et al. 1994; Boothroyd et al. 1994). Studies by Nittler et al. (1994, 1997) and Choi, Wasserburg, \& Huss (1999) found some oxide grains with very low or essentially no ${ }^{18} \mathrm{O}$. It was shown that such low ${ }^{18} \mathrm{O} /{ }^{16} \mathrm{O}$ could result from $\mathrm{HBB}$ (Boothroyd, Sackmann, \& Wasserburg 1995), but only in stars with masses 4 $M_{\odot}<M<8 M_{\odot}$. However, the data on many oxide grains were found to lie in a region of the ${ }^{17} \mathrm{O} /{ }^{16} \mathrm{O}$ and ${ }^{18} \mathrm{O} /{ }^{16} \mathrm{O}$ diagram that is not accessible by HBB. Direct observations of oxide molecules in the photospheres of some red giants had for some time indicated similar oxygen isotopic compositions, albeit with very large uncertainties (Harris et al. 1985, 1987). Similar modifications of oxygen isotopes were subsequently confirmed with a higher level of confidence in circumstellar envelopes (Kahane et al. 1992).

Discrepancies between oxide grain compositions and the compositions that result from simple dredge-up in low-mass stars were explained by the hypothesis of extra mixing of material through the zone of radiative energy transport above the hydrogen shell - referred to as "deep mixing" or "cool bottom processing" (CBP). Such a mixing process is not treated quantitatively in any stellar model with a stable and totally noncirculating radiative zone, but it cannot be ruled out on physical grounds; this extra mixing has been suggested by many authors (see below). Several abundance peculiarities besides the oxygen problem are explained by introducing CBP during at least the RGB phase of evolution. Specifically, observed patterns of ${ }^{7} \mathrm{Li}$ production and destruction and of ${ }^{13} \mathrm{C}$ production on the $\mathrm{RGB}$ require $\mathrm{CBP}$ (Gilroy \& Brown 1991; Charbonnel 1995; Boothroyd \& Sackmann 1999; Gratton et al. 2000). CBP also explains the evolution in time of the carbon isotope abundances along the RGB (Denissenkov \& Weiss 1996; Cavallo, Sweigart, \& Bell 1998). The actual mechanism that causes this mixing is not known. It is often ascribed to the shear instability in differentially rotating stars (Zahn 1992; Maeder \& Zahn 1998), but other mechanisms, such as meridional circulation and convective overshoot (Sweigart \& Mengel 1979; Herwig et al. 1997 and references therein), are available and equally likely. The specific mechanism will determine the actual depth and rate of mixing, but should not otherwise matter.

Deep mixing processes were shown to be especially important in low-metallicity stars, where deep mixing may involve relatively hot $\mathrm{H}$-burning cycles. $\mathrm{CBP}$ was therefore suggested (e.g., by Langer, Hoffman, \& Sneden 1993; Weiss, Denissenkov, \& Charbonnel 2000) as an explanation for a collection of $\mathrm{CNO}, \mathrm{Ne}-\mathrm{Na}$, and $\mathrm{Mg}-\mathrm{Al}$ anomalies observed in low-metallicity stars (reviewed in, e.g., Kraft 1994; Da Costa 1998). These studies showed that when CBP becomes deep enough to involve the $\mathrm{H}$ shell itself, the stellar evolution is significantly altered by extensive He enrichment of the envelope (Sweigart 1997). Denissenkov \& Weiss (2001), investigating elemental abundance anomalies in the globular clusters $\omega$ Cen and M4, found that at least some of the observed variations in $[\mathrm{Al} / \mathrm{Fe}]$ could result from $\mathrm{CBP}$ occurring in RGB stars. These workers used a diffusive model of extra mixing with rate and depth of mixing determined to fit observational trends. They demonstrated the possibility of ${ }^{26} \mathrm{Al}$ production in $\mathrm{RGB}$ stars undergoing $\mathrm{CBP}$, at least in 
low-metallicity stars and with particular parameters. Very recently, observations of planetary nebulae were shown to require the further addition of $\mathrm{CN}$-cycle products into the envelope during the third dredge-up (TDU) phase, even in stars with masses that are too low to permit HBB (Péquignot et al. 2000). The destruction of ${ }^{3} \mathrm{He}$ that should accompany CBP would also help explain the apparently weak evolution of the Galactic ${ }^{3} \mathrm{He}$ abundance since the big bang (Wasserburg, Boothroyd, \& Sackmann 1995, hereafter WBS; Balser et al. 1999; Gloeckler \& Geiss 1998; Bania, Rood, \& Balser 2002).

For all the above reasons, CBP is now generally accepted as a significant process occurring in a second mixing episode late in the evolution of red giants (see, e.g., Boothroyd \& Sackmann 1999; Gratton et al. 2000). The main restriction on CBP is that there be no abrupt increase in mean molecular weight anywhere between the convective envelope and the hydrogen shell to prevent mixing (Charbonnel, Brown, \& Wallerstein 1998; Boothroyd \& Sackmann 1999). This condition is known to be satisfied in $M<2 M_{\odot}$ stars on the RGB, at luminosities above that of the "bump" in the RGB luminosity function. It should also be satisfied throughout the subsequent AGB phase - and certainly during the thermally pulsing AGB (TP-AGB) phases-for all low-mass stars. From all these considerations, CBP therefore appears to be both possible and necessary in the evolution of low-mass stars.

\section{THE MODEL}

The general structure and evolution of AGB stars have been studied extensively (for a thorough introduction see J. Lattanzio 2001). ${ }^{1}$ The structure of a low-mass star during this phase of evolution is schematically as follows (see Fig. 1): In the center is a degenerate carbon/oxygen core. Outside this is a helium region that cannot support steady nuclear burning. The next layer is a thin $\left(<10^{-4} M_{\odot}\right)$ region where hydrogen burning takes place, providing essentially all of the star's luminosity. The outward advance of the $\mathrm{H}$ shell adds to the He region, which periodically undergoes nuclear burning ("thermal pulses") in short ( 200 yr) bursts separated by long $(\sim 30,000 \mathrm{yr})$ intervals. The $\mathrm{H}$ shell and the region immediately above it $\left(<10^{-3} M_{\odot}\right.$ in total $)$ are stable against convection, and we call these together the "radiative zone." Finally, the outer region of the star is a large, fully convective envelope. When mass loss is included as described below, this envelope has a mass of $\sim 0.9 M_{\odot}$ at the start of the TP-AGB and shrinks as the $\mathrm{H}$ shell advances outward and as the star sheds envelope material in a wind. Late in the AGB evolution, each thermal pulse is followed by a brief disruption of the structure just described, during which material that has seen nuclear processing in the $\mathrm{H}$ and $\mathrm{He}$ shells is mixed into the envelope. This mixing is called "the third dredge-up" (see review by BGW99). In the above description, the radiative zone contains no circulation. We treat the problem of extra mixing following WBS. Here a slow circulation is added by imposing transport of envelope material at a mass-flow rate $\dot{M}$ down to some depth inside the radiative zone specified by temperature $T_{P}$

\footnotetext{
${ }^{1}$ John Lattanzio's on-line stellar evolution tutorial is available at http://www.maths.monash.edu.au/ johnl/stellarevoln.
}

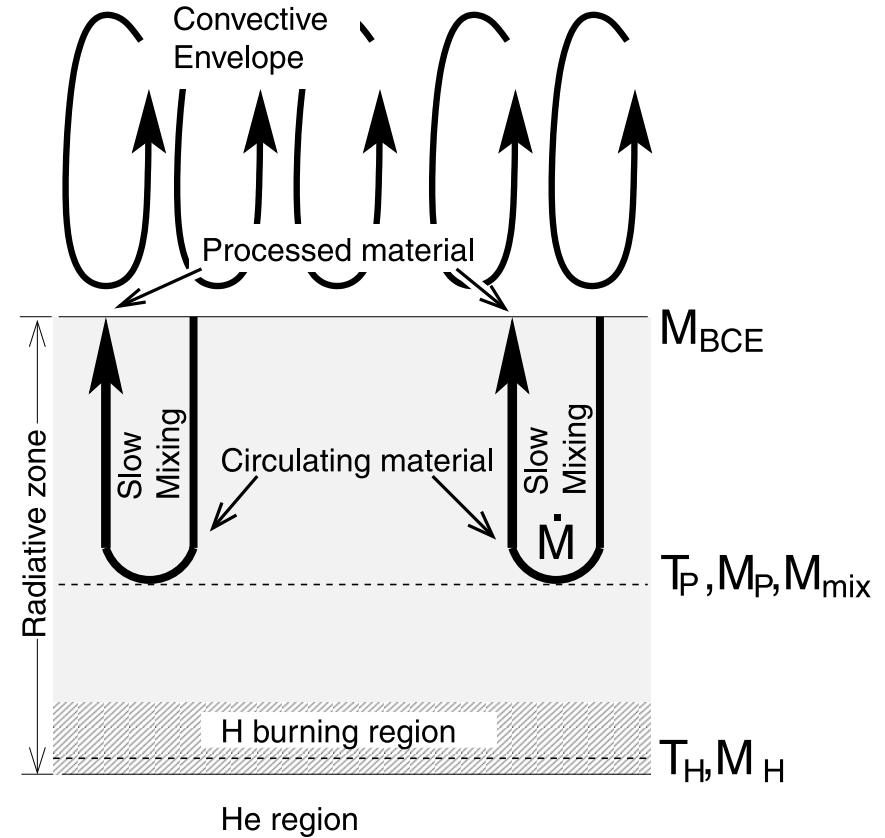

$\mathrm{C} / \mathrm{O}$ core

FIG. 1.-Schematic diagram of the CBP model. Material taken from the envelope circulates slowly down into the radiative zone, where it undergoes nuclear processing at temperatures near $T_{P}$. It then returns to the envelope, where it is rapidly mixed with the other envelope material. Relative thicknesses of the various layers do not reflect physical dimensions.

and then back to the envelope. Typically, a small amount of nuclear processing takes place in the circulating material.

We use a numerical model of an AGB star with solar initial composition and a $1.5 M_{\odot}$ initial mass. The model was computed by Straniero et al. (1997) using the FRANEC code to follow the full evolution of the star from the zeroage main sequence through the TP-AGB phase, with no mass loss. The corresponding loss of envelope mass was subsequently computed using the Reimers (1975) prescription as discussed by Busso et al. (2001). The choice $\eta=1$ for the free parameter of the Reimers formula was adopted. Details of the mass loss and dredge-up are relatively unimportant for our conclusions: runs of the code at a fixed envelope mass of $0.5 M_{\odot}$ produced the same results to $\sim 10 \%$, and variations in dredge-up efficiency are discussed below. The detailed evolution on the AGB of the above model is summarized in Figure 2 and Table 1. We refer to the detailed structure and evolution of this model, including both dredge-up and mass loss, as the stellar evolution model, SEM(1.5). For our calculations, we assume that extra mixing may be modeled by studying circulation inside the radiative-zone structures previously computed in the $\operatorname{SEM}(1.5)$ model. The nuclear processing in CBP depends mainly on the variation of temperature $T$ and density $\rho$ as functions of position within these radiative-zone structures and of time $t$. These structures are the environment within which the circulating material moves and is processed. The $\operatorname{SEM}(1.5)$ model also provides us with the timescales for AGB evolution, the rate of mass loss from the envelope, and 


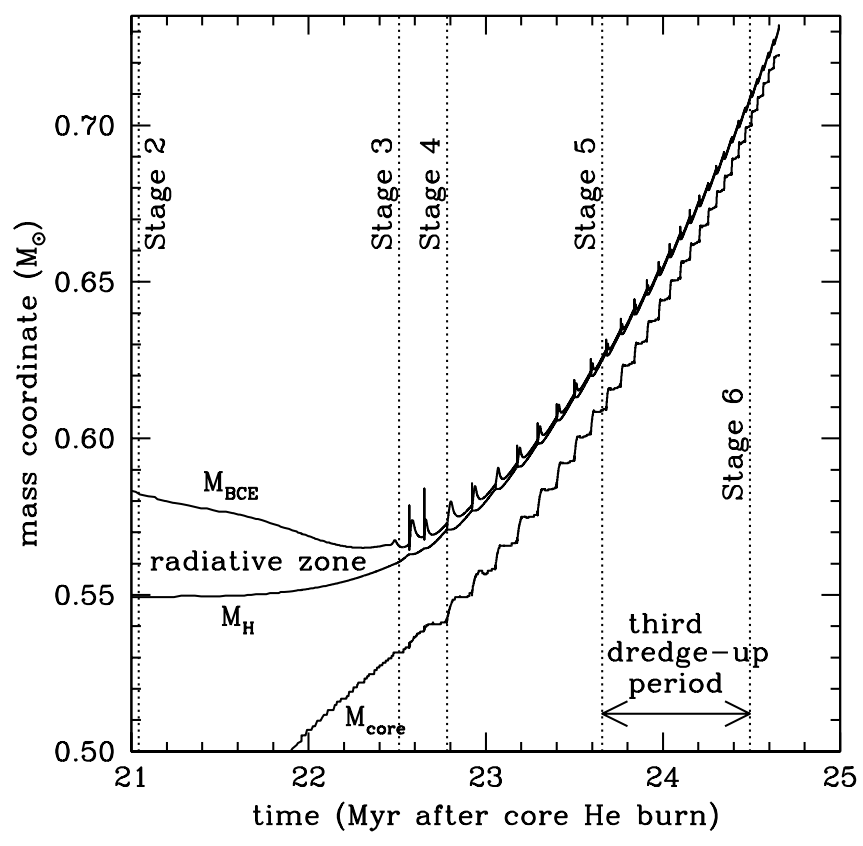

FIG. 2.-Evolution of the boundary of the $\mathrm{C} / \mathrm{O}$ core $M_{\text {core }}$, location of the $\mathrm{H}$ shell $M_{\mathrm{H}}$, and convective-envelope boundary $M_{\mathrm{BCE}}$ for the $1.5 M_{\odot}$ solar metallicity model of Straniero et al. [1997; SEM(1.5)], as functions of time since the He flash. Vertical lines indicate times selected to provide radiative-zone structures for our CBP calculations (see Table 1).

the changes to envelope composition due to TDU. The SEM(1.5) model exhibits TDU after the 10th thermal pulse and after each subsequent thermal pulse until the 26th (the interval from stage 5 to stage 6 in Fig. 2 and Table 1; two subsequent pulses produce negligible dredge-up). This dredge-up brings large quantities of newly produced carbon and of $s$-process nuclides to the envelope, and as a result, the envelope of $\operatorname{SEM}(1.5)$ with no $\mathrm{CBP}$ has $\mathrm{C} / \mathrm{O}>1$ from the 22nd pulse onward. The envelope begins $\mathrm{CBP}$ with a composition reflecting earlier first dredge-up, as discussed below for individual nuclides.

It is useful in discussing CBP to distinguish between three types of material. "Envelope material" $(E)$ makes up the convective envelope and has abundance (in moles per gram) $Y_{i}^{E}$ of nuclide $i$. "Circulating material" $(C)$ is that material moving through the radiative zone. It starts out as a representative sample of the envelope at a given time and then moves down through the radiative zone and back as shown in Figure 1. Abundances $Y_{i}^{C}$ in the circulating material are changed by $(p, \gamma)$ - and $(p, \alpha)$-reactions, as well as $\beta$-decays along the flow path. The circulating material just before returning to the envelope is the "processed material" $(P)$, with composition denoted $Y_{i}^{P}$. We assume that mixing of processed material with the convective envelope is instantaneous.

The numerical CBP model follows the envelope abundances as material descends into the radiative zone and is replaced by newly processed material. This corresponds to integrating

$$
\dot{Y}_{i}^{E}=\frac{\dot{M}}{M_{E}}\left(Y_{i}^{P}-Y_{i}^{E}\right)
$$

as $M_{E}$ (mass of the stellar envelope), $Y_{i}^{P}$, and $Y_{i}^{E}$ evolve together. The quantity $Y_{i}^{P}$ is computed by following the position of a parcel of material as it starts at the bottom of the envelope, circulates downward through the $\operatorname{SEM}(1.5)$ stellar structure, and finally returns to the envelope with composition $Y_{i}^{P}$. The stellar structure and $\dot{M}$ specify as functions of time the conditions of $T$ and $\rho$ seen by this material, and the numerical code integrates a nuclear reaction network (rates from Angulo et al. 1999; discussed in the Appendix) with these changing conditions. We do not calculate the detailed evolution by following every detail of development of the radiative zone with time. For a given calculation, we choose one of the times from the SEM(1.5) evolution listed in Table 1 (we call this a "stage"), and we hold the radiative-zone structure constant as specified by this stage. This amounts to an approximation that the processing is independent of the evolution of the radiative zone for the duration of CBP. A check of this assumption is given in $\S 3$. We consider the dominant extra processing to take place when the H-shell temperature $T_{\mathrm{H}}$ (defined as the temperature where the maximum energy generation takes place) exceeds $\log T_{\mathrm{H}} \gtrsim 7.7$ and when TDU is active. This is restricted to the last $2 \times 10^{6} \mathrm{yr}$ of $\mathrm{AGB}$ evolution of the 1.5 $M_{\odot}$ star. When considering CBP during the interval between stages 5 and 6 , we use the radiative-zone structure from stage 5, and we include TDU by interrupting the evolution at the times of the thermal pulses and altering the envelope abundances to reflect dredge-up as computed for SEM(1.5). The CBP code is restarted after each pulse, with the new composition as the initial state. We refer to the results of this calculation as the "numerical model." We also present analytical arguments to interpret the output. After an initial examination of the conditions necessary for ${ }^{26} \mathrm{Al}$ production and incorporation into grains, we confine ourselves to evolution from stage 5 to stage 6 , when TDU is active, and we use the radiative-zone structure from stage 5.

TABLE 1

Stages of AGB Evolution Considered for Cool Bottom Processing

\begin{tabular}{|c|c|c|c|c|c|}
\hline Stage & $\begin{array}{l}\text { Start Time } \\
\text { (Myr) }\end{array}$ & Description & $\log T_{\mathrm{H}}$ & $\begin{array}{c}\Delta M \\
\left(M_{\odot}\right)\end{array}$ & $\begin{array}{c}M_{E} \\
\left(M_{\odot}\right)\end{array}$ \\
\hline $1 \ldots \ldots \ldots \ldots \ldots \ldots \ldots$ & 0 & End core He burning & 7.423 & 0.376 & 0.587 \\
\hline 2. & 21.04 & Before $\mathrm{H}$-shell reignition & 7.456 & 0.0329 & 0.900 \\
\hline .............. & 22.47 & Before first pulse & 7.679 & $5.18 \times 10^{-3}$ & 0.862 \\
\hline $4 \ldots \ldots \ldots \ldots \ldots \ldots \ldots$ & 22.78 & After two mild pulses & 7.740 & $2.02 \times 10^{-3}$ & 0.887 \\
\hline $5 \ldots \ldots \ldots \ldots \ldots \ldots \ldots$ & 23.66 & Before 10th pulse (start TDU) & 7.797 & $8.65 \times 10^{-4}$ & 0.741 \\
\hline $6 \ldots \ldots \ldots \ldots \ldots \ldots \ldots$ & 24.49 & After 26th pulse (end TDU) & 7.834 & $3.17 \times 10^{-4}$ & 0.242 \\
\hline
\end{tabular}

Notes.- "Start time" is in years after the end of core He burning. The term $\Delta M$ is the thickness of the radiative zone starting at the bottom of the $\mathrm{H}$ shell. The numerical model is based on stage 5 , assuming a constant structure for the radiative zone for the interval until stage 6 . 
All of the results from the numerical model shown in the figures correspond to these calculations.

A fully self-consistent model of CBP using this "conveyor belt" model would involve calculating the abundances of the nuclides in the envelope at all times and following the evolution of stellar structure as conditions in the radiative zone change, as the $\mathrm{H}$ shell advances into regions that have been processed by CBP, and as dredge-up and mass loss change the envelope. The most extreme assumption that we have made is that a radiative-zone structure from a fixed time provides a reasonable approximation to the radiative-zone structure for the next few $10^{5} \mathrm{yr}$. We find below that this is true during the TP-AGB (excluding very brief interruptions by thermal pulses), and that $\mathrm{C}, \mathrm{N}$, and $\mathrm{O}$ isotopes are insensitive to the specific radiative-zone structure chosen. We show that for ${ }^{26} \mathrm{Al}$ there is a strong dependence on this structure, dominantly due to the evolving $T_{\mathrm{H}}$. It is shown that there are nonetheless clear general rules for ${ }^{26} \mathrm{Al}$ that adequately describe the system. Note that the conditions of $T$ and $\rho$ in the envelope play no role in this problem since no nuclear processing occurs there.

The radiative zone is only $8.6 \times 10^{-4} M_{\odot}$ thick at stage 5 , so if the envelope has a convective overturn time of $\sim 1 \mathrm{yr}$ and the radiative zone turns over much less frequently than the envelope, then $\dot{M}<10^{-4} M_{\odot} \mathrm{yr}^{-1}$, roughly. The other important constraint is that stellar energy generation not be disrupted too much, or approximately that $T_{P}<T_{\mathrm{H}}$. In principle, $\dot{M}$ does not completely specify the speed of matter circulation. The speeds of the upward and downward streams may be expressed as in WBS by $f_{u}$ and $f_{d}$, respectively, the fraction of the area at fixed radius occupied by the upward and downward streams. We have fixed both parameters at $f_{u}=f_{d}=f=0.5$, and they do not have any significant effect for fixed $f_{u}+f_{d}$ (see WBS). For each calculation we fix a value of $\dot{M}$ and a maximum depth of penetration in the radiative zone corresponding to a temperature $T_{P}$. There are no other independent parameters in the calculation.

\section{1. ${ }^{26} \mathrm{Al}$}

We first consider ${ }^{26} \mathrm{Al}$, which is the simplest case. The long-lived ground state of ${ }^{26} \mathrm{Al}$ is produced in the circulating material by the process ${ }^{25} \mathrm{Mg}(p, \gamma)^{26} \mathrm{Al}$ and destroyed by ${ }^{26} \mathrm{Al}(p, \gamma)^{27} \mathrm{Si}$. The ${ }^{26} \mathrm{Al}$ abundance $Y_{26}^{C}$ in a sample of circulating material starts with the current composition of the envelope and then evolves during the time that it circulates through the radiative zone according to

$$
\dot{Y}_{26}^{C}=Y_{25}^{C} \lambda\left[{ }^{25} \operatorname{Mg}(p, \gamma)\right]-Y_{26}^{C} \lambda\left[{ }^{26} \mathrm{Al}(p, \gamma)\right],
$$

where $\lambda[\mathrm{rxn}]=\rho N_{\mathrm{A}}\langle\sigma v\rangle Y_{\mathrm{H}}^{C}$ for the reaction labeled "rxn," $\rho$ is mass density, $N_{\mathrm{A}}$ Avogadro's number, $\langle\sigma v\rangle$ the thermally averaged reaction rate, $Y_{25}^{C}$ the ${ }^{25} \mathrm{Mg}$ abundance, and $Y_{\mathrm{H}}^{C}$ the hydrogen abundance. The quantities on the righthand side of this expression evolve as the material's position in the radiative zone changes. The destruction term is negligible for two reasons: (1) for the presently recommended rates (see the Appendix; Angulo et al. 1999), the destruction cross section is 2 orders of magnitude smaller than the production cross section, and (2) $Y_{26}^{C}$ never builds up to a level comparable to $Y_{25}^{C}$. The rate of ${ }^{26} \mathrm{Al}$ decay during the time spent circulating through the radiative zone is negligible.
For most values of $\dot{M}$ and for $T_{P}<T_{\mathrm{H}}$, the ${ }^{26} \mathrm{Al}$ production rate is low enough that only a small fraction of the ${ }^{25} \mathrm{Mg}$ burns on a single pass through the radiative zone. As a result, $Y_{25}^{C}$ is almost constant, and the amount of ${ }^{26} \mathrm{Al}$ in the processed material is approximately

$$
\begin{gathered}
Y_{26}^{P}\left(t_{f}\right)=Y_{26}^{E}\left(t_{i}\right)+\int_{t_{i}}^{t_{f}} Y_{25}^{C} \lambda\left[{ }^{25} \mathrm{Mg}(p, \gamma)\right] d t, \\
Y_{26}^{P}\left(t_{f}\right)=Y_{26}^{E}\left(t_{i}\right)+2 f \dot{M}^{-1} Y_{25}^{E} Y_{\mathrm{H}}^{E} \int_{M_{P}}^{M_{\mathrm{BCE}}} \rho N_{\mathrm{A}}\langle\sigma v\rangle d m,
\end{gathered}
$$

where we have taken advantage of the relationship between $\dot{M}$ and the time-dependent position of the circulating matter to write the integral in mass coordinates with no explicit reference to time. The mass coordinate $M_{P}$ is where $T=T_{P}$, and $M_{\mathrm{BCE}}$ is the bottom of the convective envelope (see Fig. 1); the time integral begins at the time $t_{i}$ when the circulating material leaves the envelope and ends at time $t_{f}$ when it returns to the envelope. (The factor of 2 arises because the circulating material passes each point twice: once on the way down and once on the way back up.) Note that $Y_{26}^{P}\left(t_{f}\right)-Y_{26}^{E}\left(t_{i}\right)$ does not change with time, except to the extent that stellar evolution changes the path integral.

The abundance of ${ }^{26} \mathrm{Al}$ in the envelope reflects the addition of processed material with the ${ }^{26} \mathrm{Al}$ abundance $Y_{26}^{P}$ to the envelope of mass $M_{E}$, competing against free decay with mean lifetime $\tau_{26}=1.0 \times 10^{6} \mathrm{yr}$ and removal of material into the downward stream:

$$
\begin{aligned}
\dot{Y}_{26}^{E} & =\frac{\dot{M}}{M_{E}}\left(Y_{26}^{P}-Y_{26}^{E}\right)-\tau_{26}^{-1} Y_{26}^{E} \\
& =2 f M_{E}^{-1} Y_{25}^{E}(0) Y_{\mathrm{H}}^{E} \int_{M_{P}}^{M_{\mathrm{BCE}}} \rho N_{\mathrm{A}}\langle\sigma v\rangle d m-\tau_{26}^{-1} Y_{26}^{E} \\
& =P-\tau_{26}^{-1} Y_{26}^{E},
\end{aligned}
$$

where the last two lines define the production rate $P$ per unit mass of the envelope. Note that all factors of $\dot{M}$ have canceled: the amount of processed material is proportional to $\dot{M}$, but the time it spends at high temperature is proportional to $\dot{M}^{-1}$. If the envelope mass decreases with time and the reaction path integral over the circulation stays constant, then $P$ will increase with $1 / M_{E}(t)$.

Calculations were done in the numerical model using the radiative-zone structure of stage 5, with $\mathrm{CBP}$ occurring roughly for the duration of TDU (from stage 5 until stage 6). The results for ${ }^{26} \mathrm{Al}$ are shown in Figure $3 a$ as a function of $T_{P}$. During this interval, ${ }^{26} \mathrm{Al} /{ }^{27} \mathrm{Al}=6 \times 10^{-4}$ is added gradually by normal TDU, and this amount is shown as the "TDU only" line. As argued above (see eq. [6]), there is no significant dependence on $\dot{M}$. We see that for $\log T_{P} \lesssim 7.6$ $\left(0.2\right.$ dex below $\left.T_{\mathrm{H}}\right)$ there is no significant ${ }^{26} \mathrm{Al}$ production. The term $\log \left({ }^{26} \mathrm{Al} /{ }^{27} \mathrm{Al}\right)$ rises almost linearly with $\log T_{P}$ as $T_{P}$ increases toward $T_{\mathrm{H}}$. It is evident that CBP penetrating to temperatures above $T_{P}=10^{7.6} \mathrm{~K}$ during this phase of evolution can readily produce ${ }^{26} \mathrm{Al} /{ }^{27} \mathrm{Al}$ as high as found in extreme examples of circumstellar oxide grains.

In order to understand these numerical results, we consider analytic solutions to the approximate expression in equation (6). Table 1 and Figure 2 show the selected stages of AGB evolution on which we now focus. Time 0 is taken as the state when He-core burning is just finished, and qualitative descriptions of the evolutionary stages are given in Table 1 along with characteristic parameters of the radiative 

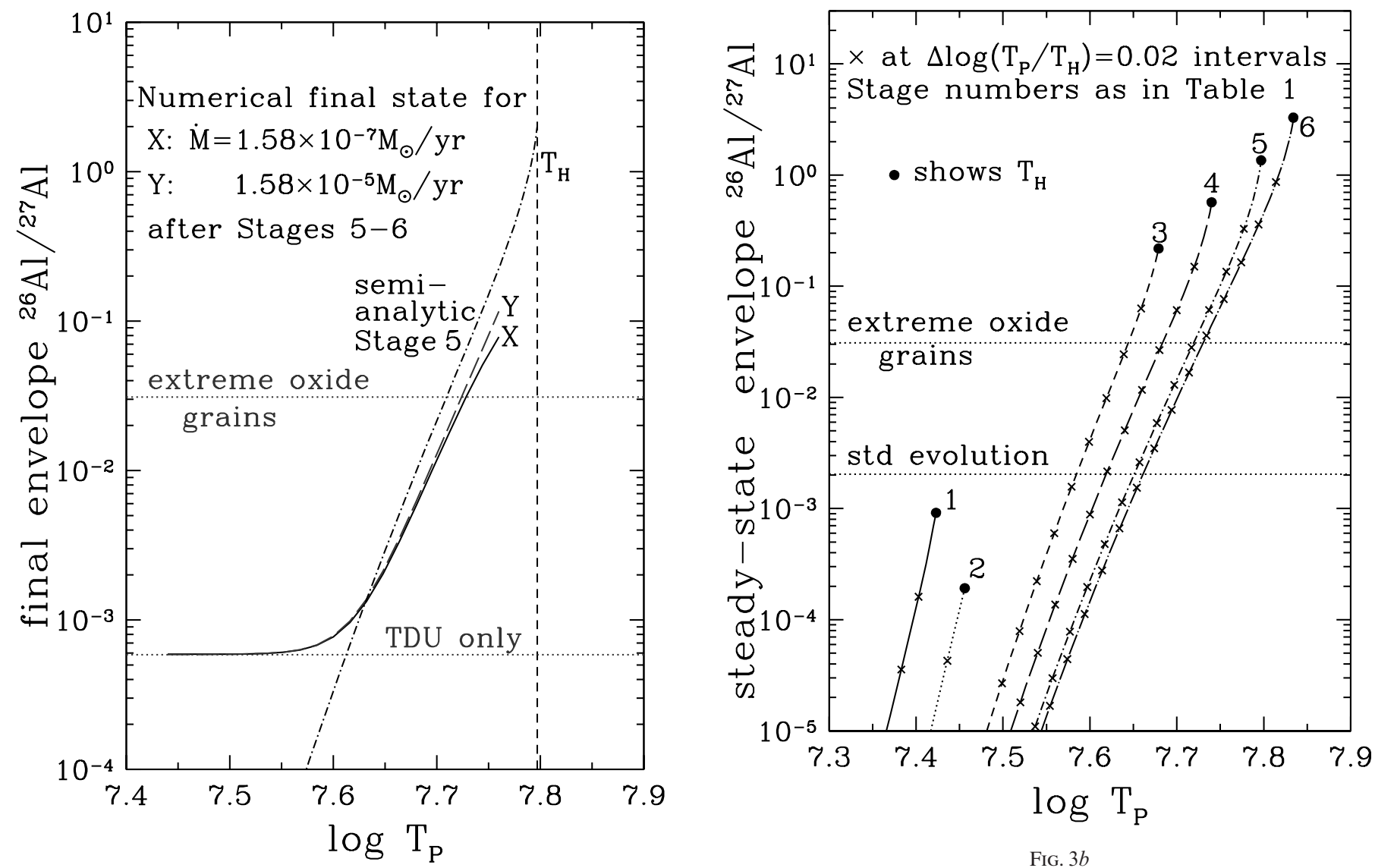

FIG. $3 b$

FIG. $3 a$

FIG. 3.-(a) Final ${ }^{26} \mathrm{Al} /{ }^{27} \mathrm{Al}$ as a function of $T_{P}$ from the numerical model running for the $8.5 \times 10^{5} \mathrm{yr}$ period during TDU (stage 5 to stage 6 ), compared with the corresponding semianalytic result for the CBP steady state abundance (panel $b$, curve 5). "Extreme oxide grains" indicates the highest value found for a meteoritic oxide grain. (b) Envelope ${ }^{26} \mathrm{Al} /{ }^{27} \mathrm{Al}$ at steady state (eq. [7]). The production rate $P$ changes as the star evolves; each curve shows the value of $\tau_{26} P / Y_{27}^{E}$ for a particular choice of radiative-zone structure taken from the stellar evolution model. Curves are labeled according to stage numbers in Table 1. The end of each curve indicates $T_{\mathrm{H}}$ at that time. The "std evolution" line indicates the level found for TDU production of ${ }^{26} \mathrm{Al}$ by BGW99.

zone. For the semianalytic treatment, we neglect mass loss and dredge-up, and we assume that the stellar structure (i.e., the structure at a selected stage from Table 1) does not change with time. Using the solution to equation (7) for a constant-mass envelope and a selected time-independent radiative-zone structure, we obtain

$$
Y_{26}^{E}=Y_{26}^{E}(0) e^{-t / \tau_{26}}+\left(1-e^{-t / \tau_{26}}\right) \tau_{26} P .
$$

Let us now consider the steady state abundance of ${ }^{26} \mathrm{Al}$ that could be achieved in an AGB star fixed at the structure from a particular evolutionary stage. The results for stage 5 are shown in Figure $3 a$ and are in good agreement with the corresponding numerical calculation. We consider the following additional times in the evolution: (1) the stellar structure at the immediate end of core He burning, (2) the middle of the early AGB, (3) just before the first thermal pulse, (4) just after the second mild pulse, and finally (6) just before the 26th thermal pulse (see Table 1 and Fig. 2). The ${ }^{26} \mathrm{Al} /{ }^{27} \mathrm{Al}$ ratios at steady state $\left(\tau_{26} P / Y_{27}^{E}\right)$ for each of these stellar stages are shown in Figure $3 b$. There is no dependence on $\dot{M}$ because of the cancellation noted above. The endpoints of the curves correspond to the extreme limiting case of $T_{P}=T_{\mathrm{H}}$. By the onset of the first thermal pulse, the production rates rise rapidly, and ${ }^{26} \mathrm{Al}$ production is possible only at these late stages. The critical parameter is $T_{P}$, which is not a priori known. Values of $\log T_{P} \gtrsim 7.6$ are required to produce substantial amounts of ${ }^{26} \mathrm{Al}$. Inspection of Figure $3 b$ shows that to achieve the same value of ${ }^{26} \mathrm{Al} /{ }^{27} \mathrm{Al}$ requires different $T_{P}$ for each choice of stellar structure (e.g., ${ }^{26} \mathrm{Al} /{ }^{27} \mathrm{Al}=0.03$ requires $\log T_{P}=7.64$ at stage $3,7.68$ at stage $4,7.72$ at stage 5 , and 7.73 at stage 6 ). However, if we had chosen $T_{P} / T_{\mathrm{H}} \sim$ constant for each of the last three stages rather than fixing $T_{P}$, then the ${ }^{26} \mathrm{Al} /{ }^{27} \mathrm{Al}$ production rate would be approximately constant (see $\S 3$ below).

In general, high levels of ${ }^{26} \mathrm{Al} /{ }^{27} \mathrm{Al}$ can be achieved by CBP, essentially independent of $\dot{M}$ but completely dependent on $T_{P}$. Thus, ${ }^{26} \mathrm{Al} /{ }^{27} \mathrm{Al}$ is a measure of the depth of penetration, independent of $\dot{M}$. Significant ${ }^{26} \mathrm{Al}$ production can occur over all the late stages, but we expect it to be dominated by production over the last $\tau_{26}$ of time before the envelope is lost. It can be seen in Figure $3 a$ that the numerical model is very close to the simple analytical calculation. Most of the small discrepancy arises because the TDU period lasts only for $8.5 \times 10^{5} \mathrm{yr}$, not long enough to reach steady state. Overall, the agreement is astoundingly good.

In summary, significant ${ }^{26} \mathrm{Al} /{ }^{27} \mathrm{Al}$ can result from $\mathrm{CBP}$ late on the AGB. The exact amount of ${ }^{26} \mathrm{Al}$ produced depends on the depth of circulation as expressed by $T_{P}$ and the duration of CBP (limited by the AGB lifetime) but only very weakly on the circulation rate $\dot{M}$. To produce ${ }^{26} \mathrm{Al} /$ 
${ }^{27} \mathrm{Al} \gtrsim 4 \times 10^{-3}$ just before the star ejects its envelope, it appears necessary that $\log T_{P}>7.67$. There is only small ${ }^{26} \mathrm{Al}$ production in the early AGB phase, and we infer that this also applies to any $\mathrm{CBP}$ during the preceding $\mathrm{RGB}$ phase because of the low $T_{\mathrm{H}}$ at that time.

\subsection{Oxygen}

We now examine the implications for the oxygen isotopes of the conditions that produce ${ }^{26} \mathrm{Al}$. In contrast to ${ }^{26} \mathrm{Al}$, ${ }^{18} \mathrm{O}$ is not produced but is destroyed. First dredge-up during the RGB phase provides the envelope with ${ }^{18} \mathrm{O} /{ }^{16} \mathrm{O}$ that is decreased from its initial value because of destruction during the main-sequence phase. This has been shown by Dearborn (1992), who recognized the significance of ${ }^{18} \mathrm{O}$ and ${ }^{17} \mathrm{O}$ as indexes of interior stellar temperatures and mixing processes. These results have been confirmed by El Eid (1994), Boothroyd \& Sackmann (1999), Gallino et al. (1998), and others. In contrast, the isotope ${ }^{17} \mathrm{O}$ is produced in the interior and is then enriched in the envelope above the initial value as a result of first dredge-up. Neither ${ }^{18} \mathrm{O}$ nor ${ }^{17} \mathrm{O}$ is affected by thermal pulses. The major isotope in the envelope, ${ }^{16} \mathrm{O}$, is essentially unchanged throughout stellar evolution.

The results of the numerical model for ${ }^{18} \mathrm{O} /{ }^{16} \mathrm{O}$ and ${ }^{17} \mathrm{O} /{ }^{16} \mathrm{O}$ are shown in Figures 4 and 5 as functions of $\dot{M}$. The curves show the final envelope composition after using the fixed radiative zone structure of stage 5 and processing

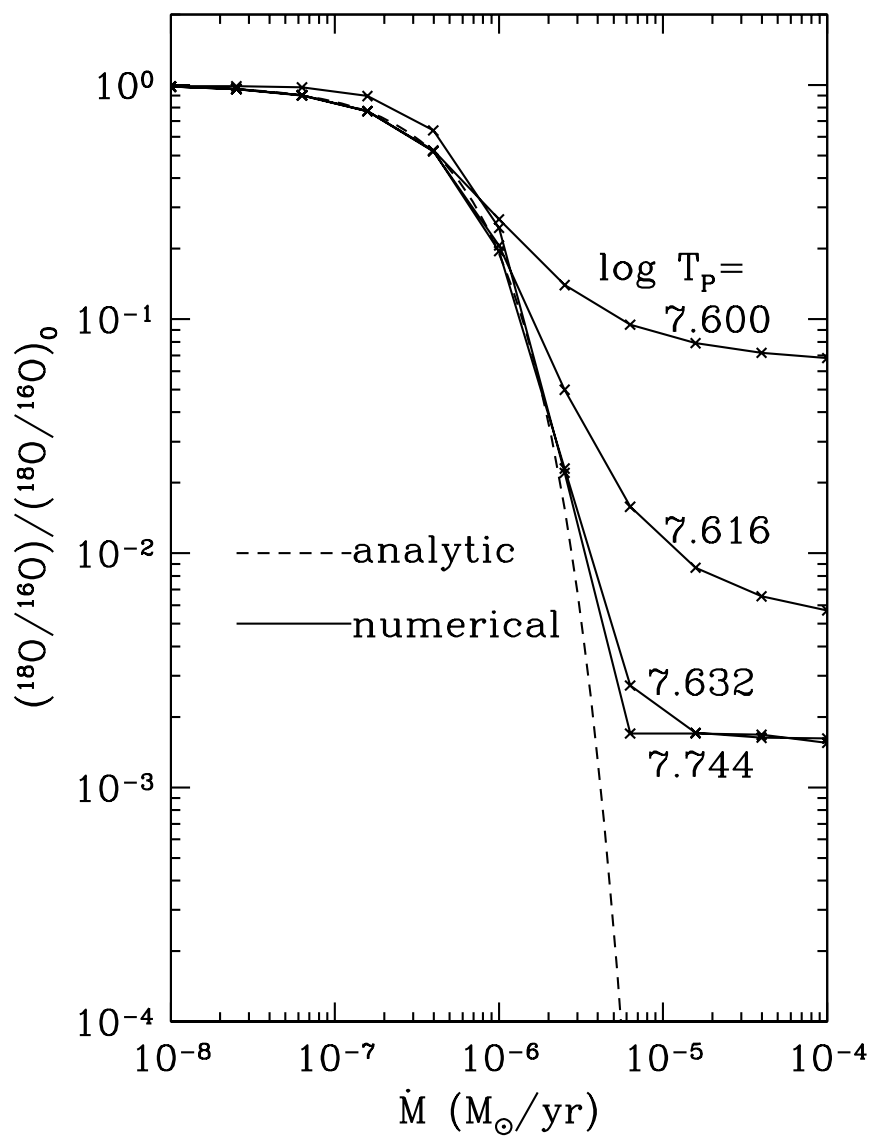

Fig. 4.-Fraction of ${ }^{18} \mathrm{O}$ surviving in the final stellar envelope, as a function of $\dot{M}$, after $8.5 \times 10^{5}$ yr. The analytic curve is based on a stellar envelope of $0.5 M_{\odot}$ and complete destruction of ${ }^{18} \mathrm{O}$ in the circulating material (eq. [13]). The solid curves correspond to processing for the same amount of time in the numerical model, at four different values of $T_{P}$.

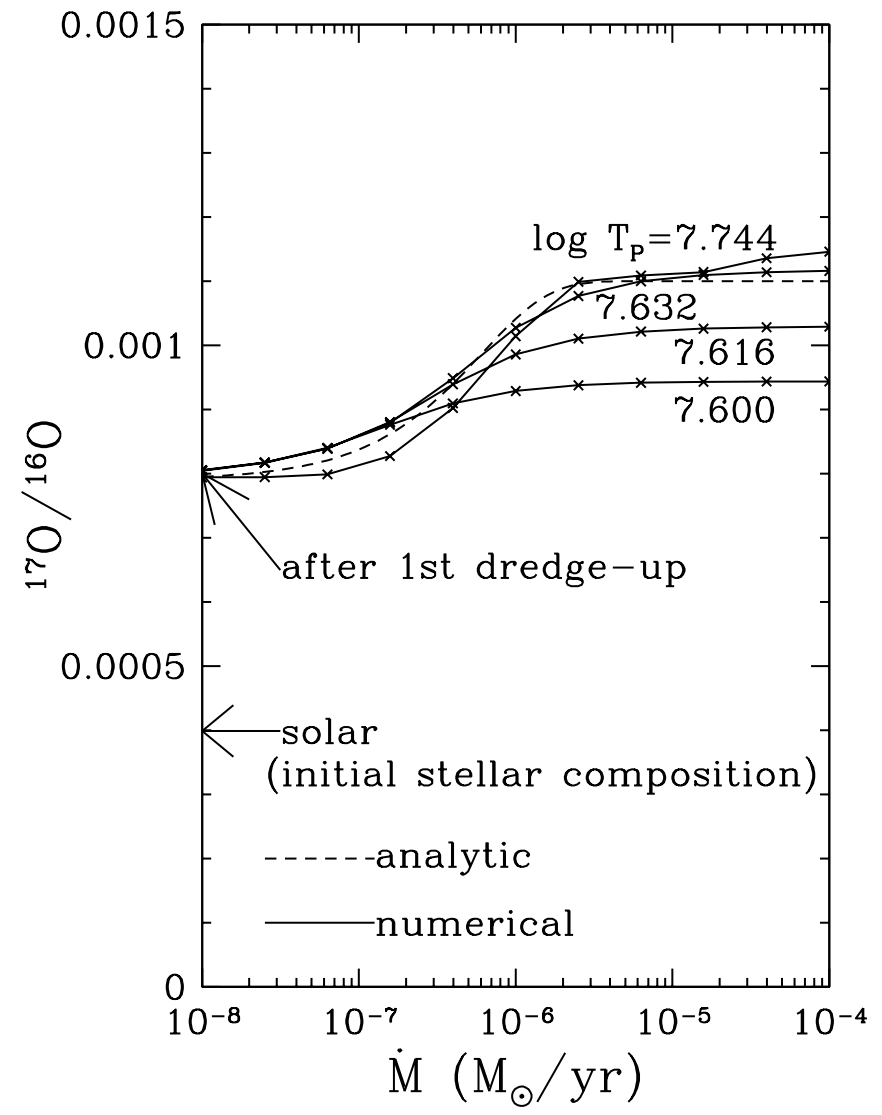

FIG. $5 .-{ }^{17} \mathrm{O} /{ }^{16} \mathrm{O}$ ratio in the final stellar envelope after processing throughout the TDU period. The analytic curve (eq. [16]) reflects an initial envelope with composition set by first dredge-up, mixing with processed material with the composition ${ }^{17} \mathrm{O} /{ }^{16} \mathrm{O}=0.0011$. The other curves are results from the corresponding numerical model, at four different values of $T_{P}$.

through the length of the TDU period. Curves for various values of $\log T_{P}$ are given. It can be seen that for $\dot{M}<10^{-7}$ $M_{\odot} \mathrm{yr}^{-1}$, there is no substantial change in ${ }^{18} \mathrm{O}$ for all $T_{P}$. This is because very little material is processed over the lifetime of the AGB for these mixing rates. For $\dot{M} \gtrsim 3 \times 10^{-7}$ $M_{\odot} \mathrm{yr}^{-1}$, there is a sharp drop-off with a magnitude depending on $T_{P}$. For rates above $3 \times 10^{-6} M_{\odot} \mathrm{yr}^{-1}$, the destruction is almost complete when $\log T_{P}>7.62$. The horizontal parts of the curves at higher $T_{P}$ are due to a small amount of ${ }^{18} \mathrm{O}$ production balancing the destruction. For ${ }^{17} \mathrm{O}$ there is a rise to higher ${ }^{17} \mathrm{O} /{ }^{16} \mathrm{O}$ by a factor of $\sim 1.5$ (dependent on $T_{P}$ ) above the value after first dredge-up. We find that if $\log T_{P}$ exceeds a low threshold, the calculation of the final composition as a function of $M$ does not change significantly. This threshold is 7.62 at stage 5 and 7.60 at stage 3 . Therefore, the results are robust, and a calculation of the full evolution with continuously changing structure and CBP would not significantly alter the results found here by assuming a fixed radiative zone.

In order to understand the results, we examine the transport equations as was done for ${ }^{26} \mathrm{Al}$. The basic results can be obtained almost quantitatively from an analytic treatment. As long as $\log T_{P}>7.62$, the evolution of ${ }^{18} \mathrm{O}$ in CBP is characterized by rapid destruction. (This applies for stage 5; other stages are characterized by analogous thresholds, never lower than 7.60 or higher than 7.65.) This destruction occurs via ${ }^{18} \mathrm{O}(p, \alpha){ }^{15} \mathrm{~N}$, with ${ }^{18} \mathrm{O}$ abundance in the circulat- 
ing material evolving according to

$$
\dot{Y}_{18}^{C}=-\lambda\left[{ }^{18} \mathrm{O}(p, \alpha)\right] Y_{18}^{C}
$$

[production via ${ }^{17} \mathrm{O}(p, \gamma){ }^{18} \mathrm{~F}\left(\beta^{+} \nu\right){ }^{18} \mathrm{O}$ is negligible]. The ${ }^{18} \mathrm{O}$ abundance in the processed material returning to the envelope is the time integral of this equation along the entire path taken by the material through the radiative zone, given by

$$
\begin{aligned}
Y_{18}^{P} & =Y_{18}^{E} \exp \left\{-\int_{t_{i}}^{t_{f}} \lambda\left[{ }^{18} \mathrm{O}(p, \alpha)\right] d t\right\} \\
& =Y_{18}^{E} \exp \left\{-2 f \dot{M}^{-1} Y_{\mathrm{H}}^{C} \int_{M_{P}}^{M_{\mathrm{BCE}}} \rho N_{\mathrm{A}}\langle\sigma v\rangle d m\right\} .
\end{aligned}
$$

The exponential factor is typically very small for even modest values of $T_{P}$; for example, this expression gives $Y_{18}^{P}=0.007 Y_{18}^{E}$ if $\log T_{P}=7.67$ and $\dot{M}=10^{-6} M_{\odot} \mathrm{yr}^{-1}$ using the radiative zone of stage 5 . The result is ${ }^{18} \mathrm{O} /{ }^{16} \mathrm{O}$ as low as $10^{-5}$ in the processed material. Higher $T_{P}$ or lower $\dot{M}$ would further reduce $Y_{18}^{P}$, as would processing slightly earlier in the TP-AGB evolution, when the threshold for destruction is lower (analogously with ${ }^{26} \mathrm{Al}$ production). For example, using the radiative zone of stage 5 with $\log T_{P}=7.69$ and $\dot{M}=10^{-6} \quad M_{\odot} \mathrm{yr}^{-1}$ gives $Y_{18}^{P}=4.2$ $\times 10^{-5} Y_{18}^{E}$. Using the radiative zone at stage 3 with $\dot{M}=10^{-6} M_{\odot} \mathrm{yr}^{-1}$, only $\log T_{P}=7.61$ is needed to produce $Y_{18}^{P}=0.007 Y_{18}^{E}$.

Because ${ }^{18} \mathrm{O}$ destruction in the processed material is so thorough, the envelope abundance of ${ }^{18} \mathrm{O}$ reflects only the removal of envelope material and its replacement with material containing essentially no ${ }^{18} \mathrm{O}$ :

$$
\dot{Y}_{18}^{E} \simeq-\frac{\dot{M}}{M_{E}} Y_{18}^{E} .
$$

The result is the exponential decay of ${ }^{18} \mathrm{O} /{ }^{16} \mathrm{O}$ with time, so if $M_{E}$ is constant,

$$
\frac{Y_{18}^{E}}{Y_{16}^{E}}=\frac{Y_{18}^{E}(0)}{Y_{16}^{E}} \exp \left(\frac{-\dot{M} t}{M_{E}}\right) .
$$

In this analytical treatment, the destruction of ${ }^{18} \mathrm{O}$ in the processed material is taken to be almost complete. This will apply to all stages of the evolution above the threshold $T_{P}$ discussed above. Hence, the resulting ${ }^{18} \mathrm{O} /{ }^{16} \mathrm{O}$ in the envelope at the end of the AGB is given by equation (13), where the only parameters are $\dot{M}, M_{E}$, and the timescale for AGB evolution. This is shown for $t=8.5 \times 10^{5} \mathrm{yr}$ (the length of the TDU period) and $M_{E}=0.5 M_{\odot}$ as the dashed curve in Figure 4. It can be seen that the semianalytic treatment is almost indistinguishable from the numerical model over most of the range of interest. The flat region at $\dot{M}>10^{-5}$ $M_{\odot} \mathrm{yr}^{-1}$ would have been obtained if we had not neglected the small ${ }^{18} \mathrm{O}$ production.

Because of the low temperature at which ${ }^{18} \mathrm{O}$ is destroyed, it is evident that low ${ }^{18} \mathrm{O} /{ }^{16} \mathrm{O}$ in the envelope is not necessarily related to ${ }^{26} \mathrm{Al}$ production. As long as $T_{P} \gtrsim 7.62$, ${ }^{18} \mathrm{O} /{ }^{16} \mathrm{O}$ in the envelope is completely independent of $T_{P}$. This is illustrated by plotting in Figure 6 for ${ }^{18} \mathrm{O} /{ }^{16} \mathrm{O}$ versus ${ }^{26} \mathrm{Al} /{ }^{27} \mathrm{Al}$ the final compositions after $\mathrm{CBP}$ running through the TDU period. Compositions were computed with the numerical model. One can see that the curves of constant $\dot{M}$ (dotted curves) are approximately curves of constant final ${ }^{18} \mathrm{O} /{ }^{16} \mathrm{O}$, while the curves of constant $T_{P}$ (dashed curves) are

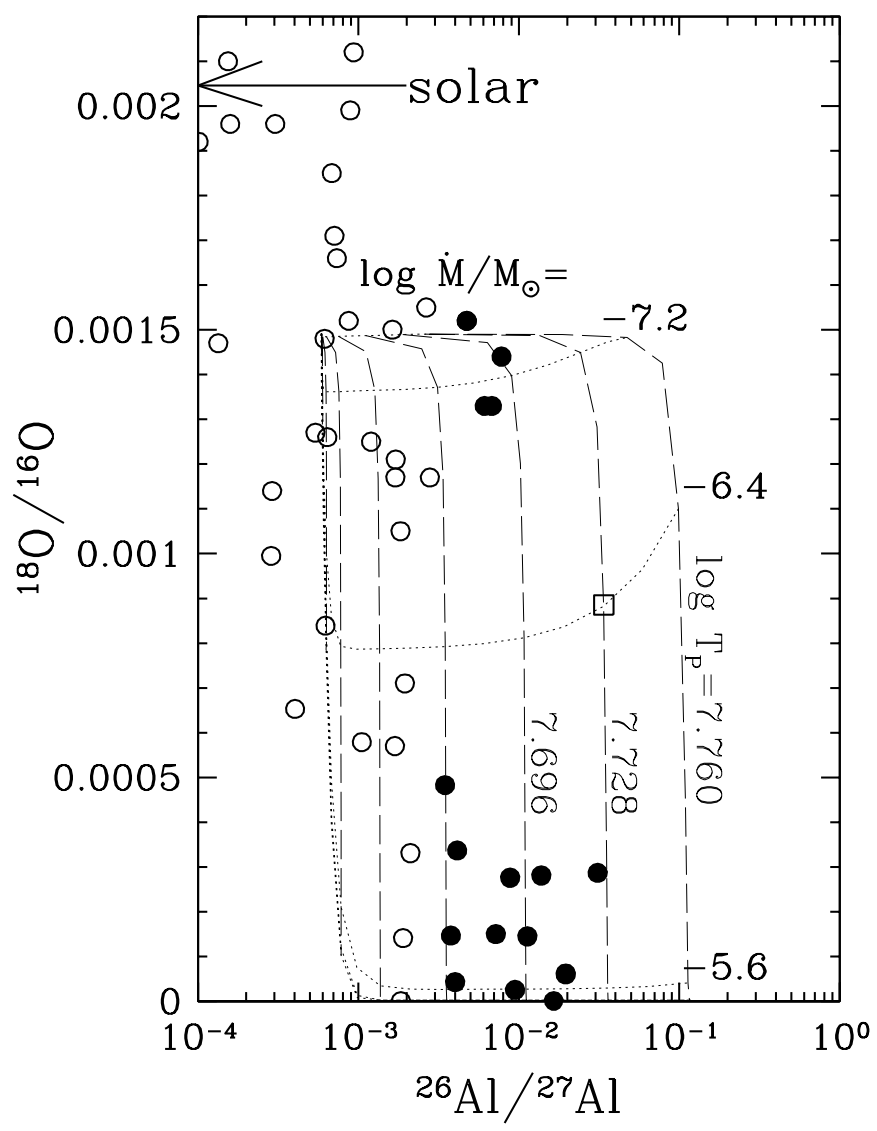

FIG. 6.-Final envelope compositions from the numerical model with the stage 5 radiative-zone structure. The grid consists of curves of constant $\dot{M}$ (dotted curves) and constant $T_{P}$ (dashed curves). Superposed over the grid are the oxide-grain data summarized in Choi et al. (1998); filled circles show those with ${ }^{26} \mathrm{Al} /{ }^{27} \mathrm{Al}>2 \times 10^{-3}$. In principle, one can read values of $\dot{M}$ and $T_{P}$ off of this and subsequent figures; for example, a grain with ${ }^{26} \mathrm{Al} /{ }^{27} \mathrm{Al}=3.5 \times 10^{-2}$ and ${ }^{18} \mathrm{O} /{ }^{16} \mathrm{O}=0.00087$ (square) corresponds to the final product of CBP with $\log T_{P}=7.728$ and $\log \left(\dot{M} / M_{\odot}\right)=-6.4$.

approximately curves of constant ${ }^{26} \mathrm{Al} /{ }^{27} \mathrm{Al}$. The departure of the constant- $\dot{M}$ curves from constant ${ }^{18} \mathrm{O} /{ }^{16} \mathrm{O}$ is explained as follows: at low $T_{P}\left(\right.$ low $\left.{ }^{26} \mathrm{Al} /{ }^{27} \mathrm{Al}\right),{ }^{18} \mathrm{O}$ destruction on a single pass through the radiative zone is incomplete; at high $T_{P}$ (high $\left.{ }^{26} \mathrm{Al} /{ }^{27} \mathrm{Al}\right)$, the situation is complicated by partial ${ }^{16} \mathrm{O}$ destruction.

The final envelope compositions are the compositions most likely to be reflected in presolar grains; the most grain formation should occur with very high mass loss at the very end of AGB evolution (Table 1). The circles in Figure 6 show the abundances found in oxide grains. It appears that the model can, in principle, explain simultaneously the ${ }^{26} \mathrm{Al}$ and ${ }^{18} \mathrm{O}$ contents of all the oxide grains found so far, and with reasonable values of $\dot{M}$ and $T_{P}$ (however, see the ${ }^{17} \mathrm{O}$ trends below). The largest value of ${ }^{26} \mathrm{Al} /{ }^{27} \mathrm{Al}$ indicates a maximum $T_{P}$ in the source stars of about $10^{7.73} \mathrm{~K}$, while a wide range in $\dot{M}$ is suggested if all the ${ }^{18} \mathrm{O} /{ }^{16} \mathrm{O}$ values are attributable to CBP. Not all grains formed from $1.5 M_{\odot}$ stars. Moreover, inferred values of $\dot{M}$ and $T_{P}$ are sensitive to exactly when CBP occurs and for how long; these values do provide qualitative insight into the actual mixing rate and depth. In any case, ${ }^{18} \mathrm{O} /{ }^{16} \mathrm{O}$ decreases at a rate dependent upon $\dot{M}$ and not $T_{P}$, so that there is no strong correlation with ${ }^{26} \mathrm{Al} /{ }^{27} \mathrm{Al}$ production, except that ${ }^{26} \mathrm{Al} /{ }^{27} \mathrm{Al}$ and ${ }^{18} \mathrm{O} /{ }^{16} \mathrm{O}$ may be modified simultaneously by CBP. Low $\dot{M}$ 
and high $T_{P}$ will produce small ${ }^{18} \mathrm{O}$ depletions and abundant ${ }^{26} \mathrm{Al}$. High $\dot{M}$ and high $T_{P}$ will produce major ${ }^{18} \mathrm{O}$ depletion and high ${ }^{26} \mathrm{Al}$. Thus, a direct correlation between ${ }^{26} \mathrm{Al} /{ }^{27} \mathrm{Al}$ and ${ }^{18} \mathrm{O} /{ }^{16} \mathrm{O}$ should not be found, although Nittler et al. (1997) and Choi et al. (1999) have suggested that it might occur on an empirical basis.

The plateau of ${ }^{17} \mathrm{O} /{ }^{16} \mathrm{O}$ at $\dot{M}>10^{-6} M_{\odot} \mathrm{yr}^{-1}$ for all $T_{P}$ seen in Figure 5 shows net increases above the dredge-up value ranging from $0 \%$ to $40 \%$. This plateau value increases with temperature but is at 0.0011 for all $T_{P} \geq 7.62$. Figure $7 a$ shows ${ }^{18} \mathrm{O} /{ }^{16} \mathrm{O}$ versus ${ }^{17} \mathrm{O} /{ }^{18} \mathrm{O}$ for the final envelope composition, at various values of $\dot{M}$ and $T_{P}$. Generally speaking, the results lie in the space between point D (composition after first dredge-up) and the endpoint B. If we use stage 3 instead of stage 5 to specify the stellar structure, this changes the results by shifting the ${ }^{17} \mathrm{O} /{ }^{16} \mathrm{O}$ endpoint $\mathrm{B}$ to 0.0016 . Results for stages 4 and 6 are similar to those of stage 5.

We now examine ${ }^{17} \mathrm{O} /{ }^{16} \mathrm{O}$ using the semianalytic treatment. In the circulating material, ${ }^{16} \mathrm{O}$ destruction is very small, and the ${ }^{17} \mathrm{O}$ abundance is governed by competing production via ${ }^{16} \mathrm{O}(p, \gamma){ }^{17} \mathrm{~F}\left(\beta^{+} \nu\right){ }^{17} \mathrm{O}$ and destruction via ${ }^{17} \mathrm{O}(p, \alpha){ }^{14} \mathrm{~N}$ :

$$
\dot{Y}_{17}^{C}=Y_{16}^{C} \lambda\left[{ }^{16} \mathrm{O}(p, \gamma)\right]-Y_{17}^{C} \lambda\left[{ }^{17} \mathrm{O}(p, \alpha)\right] .
$$

Starting from a low initial abundance, $Y_{17}^{C}$ increases as matter moves through the radiative zone until the two terms on the right-hand side of equation (14) cancel. The equilibrium ratio specified by this cancellation is determined completely by reaction rates, so that processed material has

$$
\frac{Y_{17}^{P}}{Y_{16}^{P}}=\frac{\lambda\left[{ }^{16} \mathrm{O}(p, \gamma)\right]}{\lambda\left[{ }^{17} \mathrm{O}(p, \alpha)\right]} \sim 0.0011
$$

As a result,

$$
\frac{Y_{17}^{E}}{Y_{16}^{E}} \sim \frac{Y_{17}^{P}}{Y_{16}^{P}}-\left[\frac{Y_{17}^{P}}{Y_{16}^{P}}-\frac{Y_{17}^{E}(0)}{Y_{16}^{E}(0)}\right] e^{-\dot{M} t / M_{E}} .
$$

The final envelope composition using this approximation is shown as a function of $\dot{M}$ by the dashed curve in Figure 5. As with the numerical results presented, this assumes the radiative zone of stage 5 and $t=8.5 \times 10^{5} \mathrm{yr}$. We chose $M_{E}=0.5 M_{\odot}$ for the analytic approximation. It is clear that at the condition of $\log T_{P}>7.62$ required to obtain equilibrium, the results are in close agreement with the numerical calculation. Thus, the envelope ${ }^{17} \mathrm{O} /{ }^{16} \mathrm{O}$ as a function of time reflects the replacement of envelope matter with material that has ${ }^{17} \mathrm{O} /{ }^{16} \mathrm{O} \sim 0.0011$.

The steady state ${ }^{17} \mathrm{O} /{ }^{16} \mathrm{O}$ is almost temperature independent over the temperatures characteristic of the lower part of the radiative zone late on the AGB, as shown in Figure 8. As a result, $Y_{17}^{P} / Y_{16}^{P}$ is approximately 0.0011 for all cases with $\log T_{P}>7.62$, with an uncertainty of about $30 \%$ in the ratio arising from measurement errors on the cross section
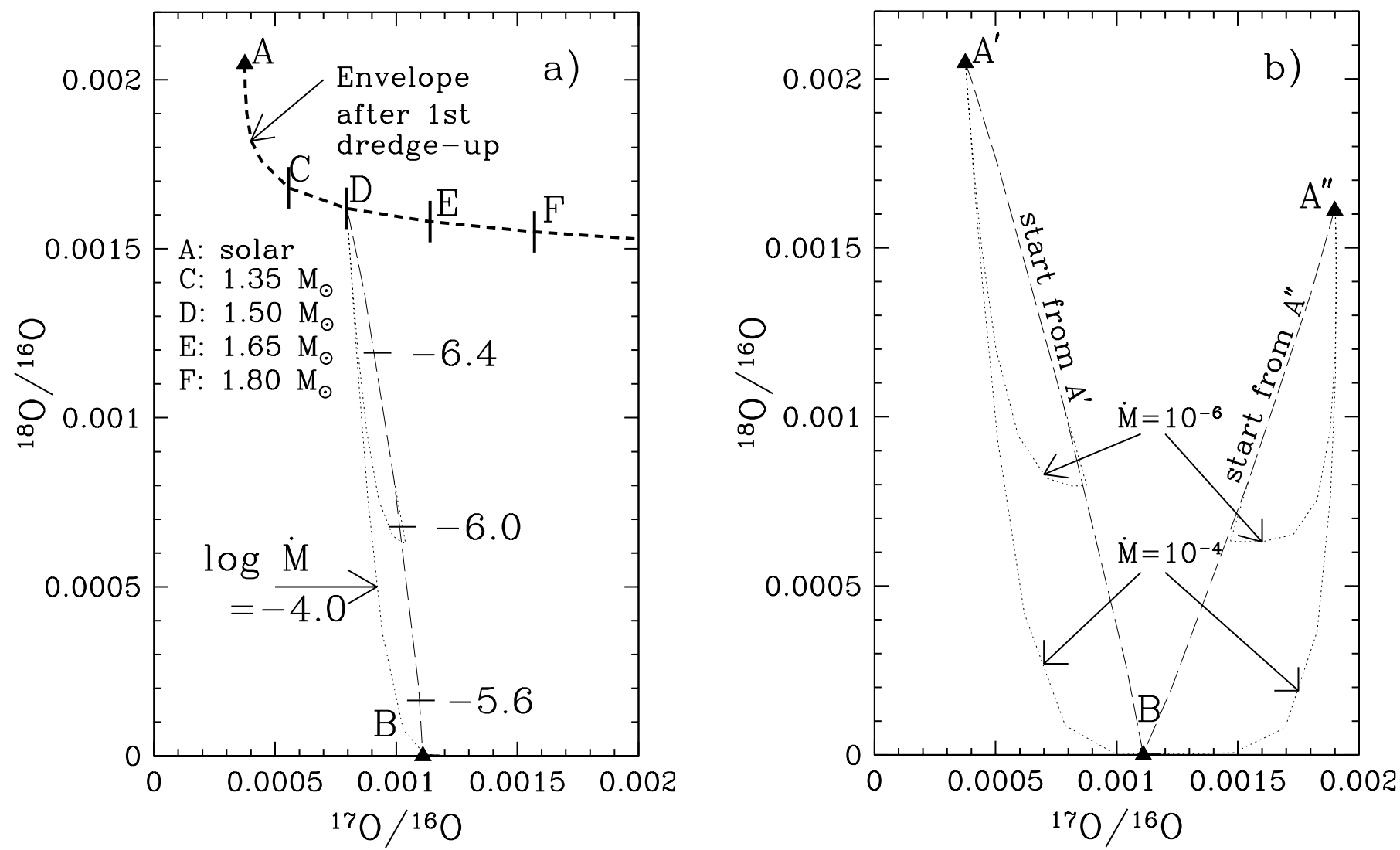

FIG. 7.-Final envelope compositions of a $1.5 M_{\odot}$ star after CBP $\left(10^{-8} M_{\odot} \mathrm{yr}^{-1} \leq \dot{M} \leq 10^{-4} M_{\odot} \mathrm{yr}^{-1} ; 7.44 \leq \log T_{P} \leq 7.76\right.$; notations as in Fig. 6). (a) Point $\mathrm{D}$ is the composition after first dredge-up. The triangle $\mathrm{B}$ is the composition reached if CBP continues for a $\operatorname{long}$ time at $\log T_{P} \gtrsim 7.62$. The calculated compositions all lie near a two-component mixing curve connecting points $\mathrm{D}$ and $\mathrm{B}$. Compositions to the left of this curve indicate incomplete processing. The thick dashed curve ACDEF indicates the results of dredge-up during the red giant phase of stellar evolution for various initial stellar masses (see WBS). (b) Trajectories resulting from $\mathrm{CBP}$ for two arbitrary stars with $\mathrm{O}$ compositions of $\mathrm{A}^{\prime}$ and $\mathrm{A}^{\prime \prime}$ after first dredge-up. Mixing lines point to the equilibrium ${ }^{17} \mathrm{O} /{ }^{16} \mathrm{O}$ value. The range of $\dot{M}$ and $T_{P}$ is the same as in $(a)$. 
inputs (Blackmon et al. 1995; Angulo et al. 1999; see also the Appendix). We note that only in the last 6 years have ${ }^{17} \mathrm{O}(p, \alpha){ }^{14} \mathrm{~N}$ cross sections been sufficiently well measured to allow us to reach this conclusion, because of the measurement of the $66 \mathrm{keV}$ resonance by Blackmon et al. (1995). At lower temperatures, the equilibrium ${ }^{17} \mathrm{O} /{ }^{16} \mathrm{O}$ increases, but the timescale to reach equilibrium also increases, so that these higher values of ${ }^{17} \mathrm{O} /{ }^{16} \mathrm{O}$ are inaccessible after stage 4 . The highest value that we found at any time on the TP-AGB is 0.0016 .

Since the envelope ${ }^{17} \mathrm{O} /{ }^{16} \mathrm{O}$ as a function of time reflects gradual replacement of envelope material with material that has fixed ${ }^{17} \mathrm{O} /{ }^{16} \mathrm{O}=0.0011$ and the processed material also has extremely low ${ }^{18} \mathrm{O} /{ }^{16} \mathrm{O}$, we now recognize the numerical results in Figure $7 a$ as mostly falling on a two-component mixing curve.

We also carried out calculations using radiative-zone structures from other stages, and the results were almost indistinguishable as long as $\log T_{P} \gtrsim 7.60$. As explained in the analytical treatment, the dominant effect for $\log T_{P}>7.62$ is to produce an envelope that is a mixture of the envelope composition after first dredge-up and the endpoint $\mathrm{B}$ that represents the equilibrium value. For all values of $\log T_{P} \gtrsim 7.62$, the numerical results lie on the mixing line at positions depending almost exclusively on $\dot{M}$, as indicated in the figure. For lower temperatures $\left(\log T_{P}<7.62\right)$, the final compositions fall in a space bounded on the one side by the mixing curve and on the other by the curve with

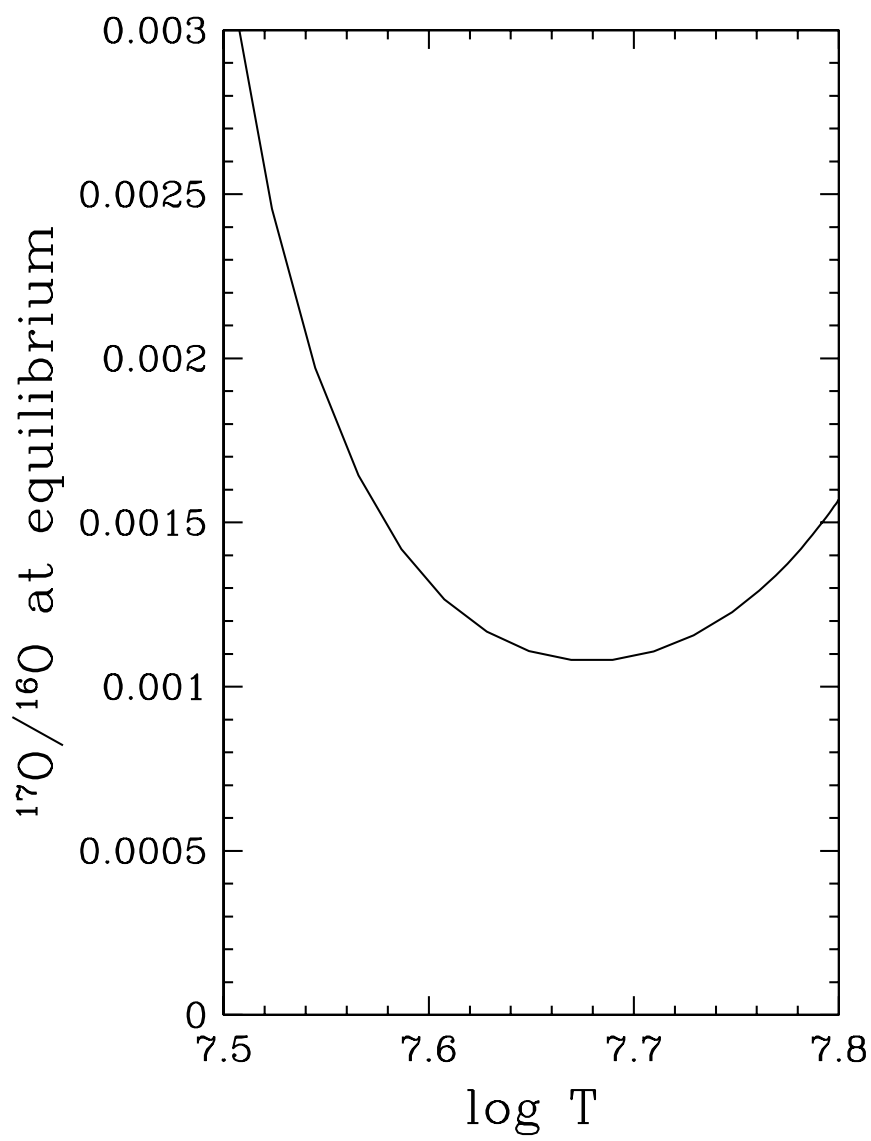

FIG. 8.-Equilibrium ${ }^{17} \mathrm{O} /{ }^{16} \mathrm{O}$ abundance ratio as a function of temperature, which depends only on the reaction rates. The high values on the left side of the graph are inaccessible in CBP because the reactions are too slow to establish equilibrium at $\log T_{P} \lesssim 7.6$ (see Table 1 ).
$\dot{M}=10^{-4} M_{\odot} \mathrm{yr}^{-1}$. More generally, since the oxygen isotopic evolution resulting from $\mathrm{CBP}$ depends on the value of ${ }^{18} \mathrm{O} /{ }^{16} \mathrm{O}$ and ${ }^{17} \mathrm{O} /{ }^{16} \mathrm{O}$ after first dredge-up and not on the detailed stellar evolution, we may readily infer the results of CBP for low-mass AGB stars of generally solar chemical abundances but with arbitrary oxygen isotopic composition: the endpoint $\mathrm{B}$ is the natural final composition for material that has been near the $\mathrm{H}$ shell in any AGB star of low mass $\left(M \lesssim 3 M_{\odot}\right)$, because all of these stars have $\mathrm{H}$-shell temperatures near $10^{7.8} \mathrm{~K}$.

The results for two initial compositions are shown in Figure $7 b$. The upper point $\mathrm{A}^{\prime}$ represents ratios in the envelope at the end of first dredge-up for some star. For $\log T_{P} \gtrsim 7.62$, the trajectory is a mixing line between $\mathrm{A}^{\prime}$ and $\mathrm{B}$, where the position depends only on $\dot{M} t$. If $\log T_{P}$ is substantially less than 7.62 but greater than 7.53 , it will be a curve that drops from $\mathrm{A}^{\prime}$ (because of ${ }^{18} \mathrm{O}$ destruction) and then swings toward $B$ as $\dot{M}$ increases. This same rule applies to $\mathrm{A}^{\prime \prime}$ or any other point, insofar as the initial stellar mass does not exceed 4-5 $M_{\odot}$, where HBB may occur (Iben \& Renzini 1983). Since the initial oxygen isotopic composition of the star that produced a particular oxide grain is not in general known, we use this construction in discussing the data on grains in $\S 4.1 .1$.

In light of the above considerations, the production of ${ }^{17} \mathrm{O} /{ }^{16} \mathrm{O}>0.0011$ by first dredge-up is explained because material in the stellar mantle is processed during the mainsequence phase at much lower temperatures than those present on the AGB. The relevant reaction rates are much slower and the equilibrium ratio larger (see Fig. 8), while the duration of the main-sequence phase is much longer than that of the AGB phase.

\subsection{Carbon}

The evolution of carbon in CBP is more complex than that of ${ }^{26} \mathrm{Al},{ }^{17} \mathrm{O}$, or ${ }^{18} \mathrm{O}$, since the nuclides discussed so far are negligibly affected by TDU. The abundance of ${ }^{12} \mathrm{C}$ in the envelope is altered at each dredge-up episode. Consequently, the ${ }^{13} \mathrm{C} /{ }^{12} \mathrm{C}$ ratio is shifted. The change in $\mathrm{C} / \mathrm{O}$ in the envelope at each thermal pulse in $\operatorname{SEM}(1.5)$ is shown in Figure $9 a$, along with the change in the mass of the envelope with time. In the numerical model, we assume that CBP starts immediately after the first thermal pulse that produces dredge-up (Table 1 and Fig. 2). This is the same calculation used for ${ }^{26} \mathrm{Al},{ }^{18} \mathrm{O}$, and ${ }^{17} \mathrm{O}$.

Figure $9 a$ shows the evolution of $\mathrm{C} / \mathrm{O}$ for $\operatorname{SEM}(1.5)$ as a function of time up until the end of TDU and includes the cases of CBP with $\dot{M}=10^{-6.0} M_{\odot} \mathrm{yr}^{-1}$ and $\log T_{P}=7.744$ for comparison. Note that $\mathrm{C} / \mathrm{O}=\left({ }^{12} \mathrm{C}+{ }^{13} \mathrm{C}\right) /{ }^{16} \mathrm{O}$. In Figure $9 b$ we show the evolution of ${ }^{12} \mathrm{C} /{ }^{13} \mathrm{C}$ in the envelope versus ${ }^{26} \mathrm{Al} /{ }^{27} \mathrm{Al}$ with time for three cases. As already stated, it is evident that high values of $T_{P}$ give high ${ }^{26} \mathrm{Al} /{ }^{27} \mathrm{Al}$. However, to obtain low ${ }^{12} \mathrm{C} /{ }^{13} \mathrm{C}$ at high ${ }^{26} \mathrm{Al} /{ }^{27} \mathrm{Al}$ requires high $\dot{M}$ (e.g., $10^{-5} M_{\odot} \mathrm{yr}^{-1}$ ). In the latter case, the ${ }^{12} \mathrm{C}$ content of the envelope is so low that every thermal pulse gives a spike in ${ }^{12} \mathrm{C} /{ }^{13} \mathrm{C}$ that is then destroyed by rapid $\mathrm{CBP}$. The final envelope compositions in terms of $\mathrm{C} / \mathrm{O}$ and ${ }^{26} \mathrm{Al} /{ }^{27} \mathrm{Al}$ are shown in Figure 10. It can be seen that the approximate boundary for $\mathrm{C} / \mathrm{O} \gtrsim 1$ is $\dot{M} \approx 10^{-6.4} M_{\odot} \mathrm{yr}^{-1}$. The relationship between ${ }^{12} \mathrm{C} /{ }^{13} \mathrm{C}$ and $\mathrm{C} / \mathrm{O}$ is shown in Figure 11. The filled triangle represents the final envelope composition of the AGB star computed by $\operatorname{SEM}(1.5)$ with no CBP. The results for CBP are shown as curves of constant $\dot{M}$, with the 

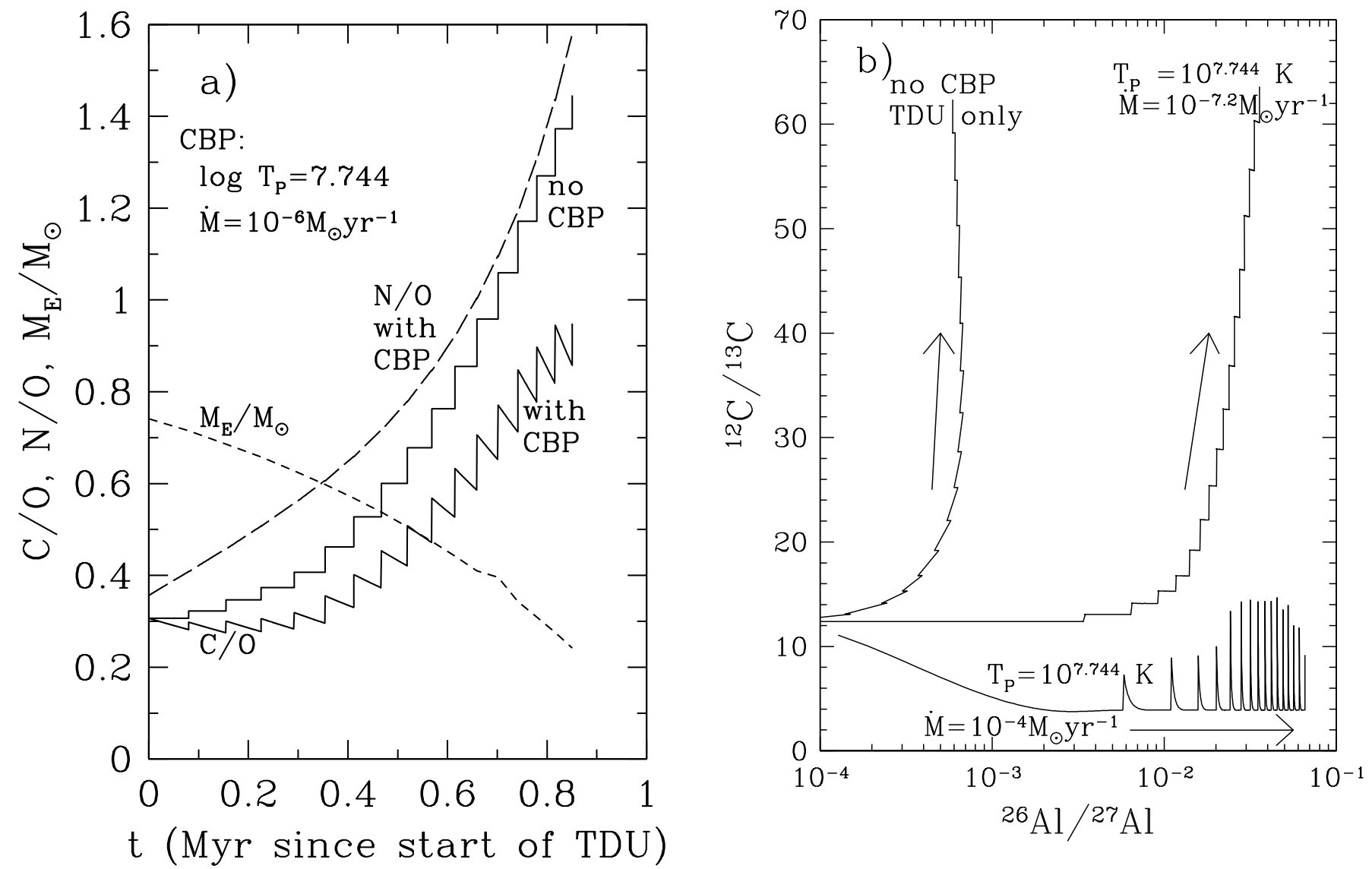

FIG. 9.-(a) Time evolution of C/O in SEM(1.5) (no CBP). Also shown is the envelope mass as a function of time. The C/O curve labeled "with CBP" is the evolution of $\mathrm{C} / \mathrm{O}$ with time for a star with CBP. The N/O curve is for the same CBP model. Note that CBP adds to N/O in the envelope at the expense of C/O. (b) Evolution of ${ }^{12} \mathrm{C} /{ }^{13} \mathrm{C}$ and ${ }^{26} \mathrm{Al} /{ }^{27} \mathrm{Al}$ with time in the numerical model of concurrent CBP and TDU, at two choices of $\dot{M}$ and compared with the no-CBP model. Arrows indicate the general direction of evolution. The jumps in composition are due to dredge-up episodes, and motion toward lower ${ }^{26} \mathrm{Al}$ reflects radioactive decay. Increasing $T_{P}$ moves the endpoints of these curves to the right. Increasing $\dot{M}$ moves the endpoints downward.

values of $\log T_{P}$ varying with position along these curves. It can be seen that to obtain both $\mathrm{C} / \mathrm{O}>1$ and low ${ }^{12} \mathrm{C} /{ }^{13} \mathrm{C}$ $(\lesssim 20)$ requires conditions that are very restrictive $\left(\log T_{P} \lesssim 7.616\right)$. Low ${ }^{12} \mathrm{C} /{ }^{13} \mathrm{C}$ may readily occur for higher $T_{P}$ but with $\mathrm{C} / \mathrm{O}<1$.

In the material circulating through the radiative zone, the ${ }^{12} \mathrm{C}$ and ${ }^{13} \mathrm{C}$ abundances are governed by the equations

$$
\begin{aligned}
& \dot{Y}_{12}^{C}=Y_{15}^{C} \lambda\left[{ }^{15} \mathrm{~N}(p, \alpha)\right]-Y_{12}^{C} \lambda\left[{ }^{12} \mathrm{C}(p, \gamma)\right], \\
& \dot{Y}_{13}^{C}=Y_{12}^{C} \lambda\left[{ }^{C} \mathrm{C}(p, \gamma)\right]-Y_{13}^{C} \lambda\left[{ }^{13} \mathrm{C}(p, \gamma)\right] .
\end{aligned}
$$

As a sample of circulating material descends into the radiative zone and its temperature rises, the negative term on the right-hand side of equation (17) grows and ${ }^{12} \mathrm{C}$ is destroyed. The ${ }^{12} \mathrm{C}$ that is destroyed is converted to ${ }^{13} \mathrm{C}$, so that quickly both the production and destruction terms in equation (18) balance. The condition that ${ }^{12} \mathrm{C} /{ }^{13} \mathrm{C}$ in this material does not change with time then gives the steady state ratio

$$
\frac{Y_{12}^{C}}{Y_{13}^{C}}=\frac{\lambda\left[{ }^{13} \mathrm{C}(p, \gamma)\right]-\lambda\left[{ }^{12} \mathrm{C}(p, \gamma)\right]}{\lambda\left[{ }^{12} \mathrm{C}(p, \gamma)\right]},
$$

which depends only on cross sections. For the temperatures in the radiative zone, it is about 3 . As processing continues, ${ }^{12} \mathrm{C}$ continues to burn, while ${ }^{12} \mathrm{C} /{ }^{13} \mathrm{C}$ remains approximately constant, producing ${ }^{14} \mathrm{~N}$. Finally, if $T_{P}$ is sufficiently high, enough ${ }^{14} \mathrm{~N}$ is made to close the $\mathrm{CN}$ cycle with the reaction chain ${ }^{14} \mathrm{~N}(p, \gamma){ }^{15} \mathrm{O}\left(\beta^{+} \nu\right)^{15} \mathrm{~N}(p, \alpha){ }^{12} \mathrm{C}$, and the amounts of ${ }^{12} \mathrm{C}$ and ${ }^{13} \mathrm{C}$ become steady with ${ }^{12} \mathrm{C} /{ }^{13} \mathrm{C} \sim 4$ and $Y_{12}^{C} \sim 0.03\left[Y_{12}^{E}(t)+Y_{13}^{E}(t)\right]$. Thus, CBP destroys ${ }^{12} \mathrm{C}$, mixing ${ }^{12} \mathrm{C}$-depleted and ${ }^{13} \mathrm{C}$-enhanced material into the envelope. This explains the strong dependence of the $\mathrm{C}$ isotopes on $\dot{M}$ : at most temperatures, $M$ sets the rate at which processed material of the new ${ }^{12} \mathrm{C} /{ }^{13} \mathrm{C}$ composition is mixed into the envelope.

The effects of CBP can be seen as follows for a given $\dot{M}$. The ratio ${ }^{12} \mathrm{C} /{ }^{13} \mathrm{C}$ first decreases with increasing $T_{P}$ because of increasing input of processed material, approaching ${ }^{12} \mathrm{C} /{ }^{13} \mathrm{C} \sim 3$. For $\log T_{P} \gtrsim 7.6$, most of the $\mathrm{C}$ is converted to ${ }^{14} \mathrm{~N}$ in the processed material, so that processed material contains only small amounts of ${ }^{12} \mathrm{C}$ and ${ }^{13} \mathrm{C}$. As a consequence, ${ }^{12} \mathrm{C} /{ }^{13} \mathrm{C}$ in the envelope remains near the value it would have without CBP. For $\dot{M} \gtrsim 10^{-6.4} M_{\odot} \mathrm{yr}^{-1}$, the processing at $\log T_{P} \gtrsim 7.6$ is so effective that a substantial amount of carbon is converted to $\mathrm{N}$, and $\mathrm{C} / \mathrm{O}$ becomes less than 1 .

\subsection{C-rich versus O-rich Envelopes}

The conversion of a large fraction of the carbon in the stellar envelope into nitrogen has important observational effects: the $\mathrm{C} / \mathrm{O}$ ratio determines whether the signs of related nucleosynthesis should be expected in $\mathrm{S}$ stars versus carbon stars, or in oxide grains versus carbon and carbide 


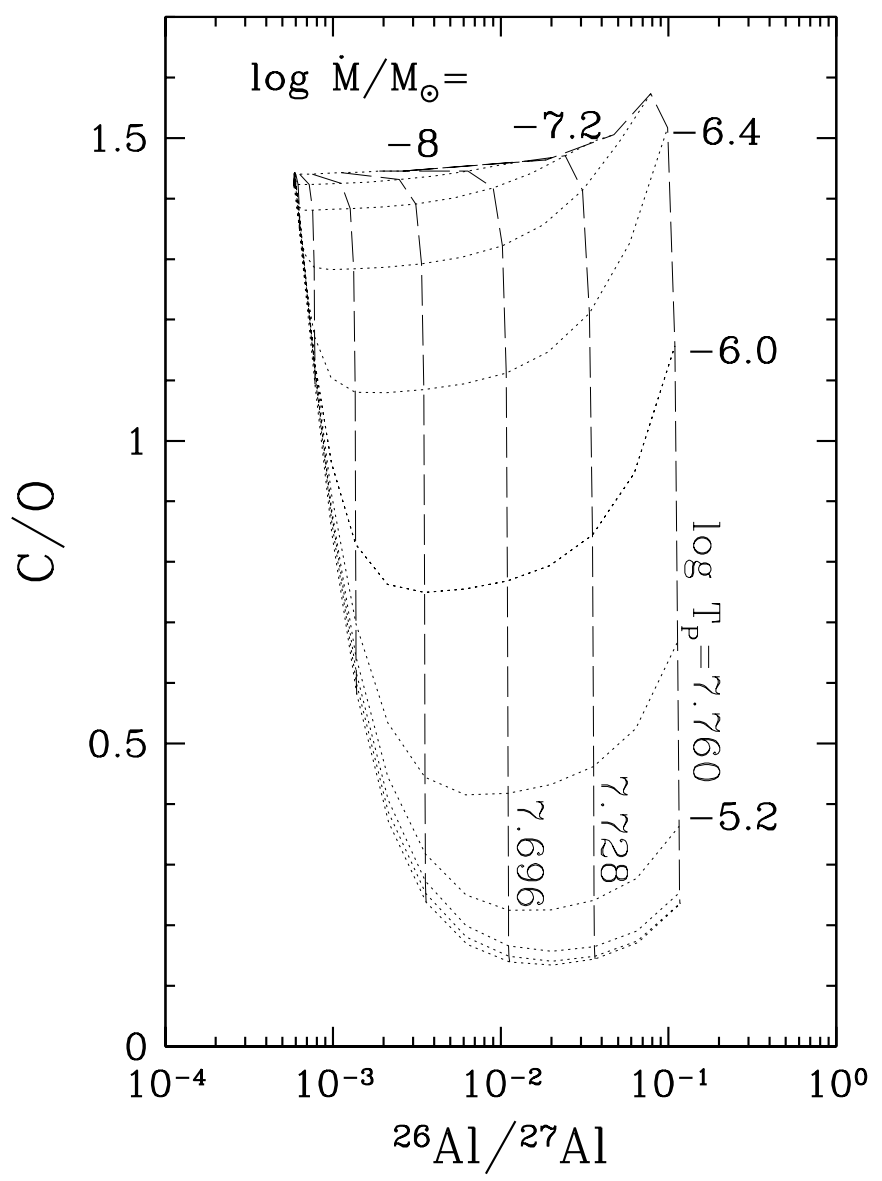

FIg. 10.-Final envelope compositions, with $7.44 \leq \log T_{P} \leq 7.76$ and $10^{-8} M_{\odot} \mathrm{yr}^{-1} \leq \dot{M}<10^{-4} M_{\odot} \mathrm{yr}^{-1}$. Note that a carbon star is produced when $\mathrm{C} / \mathrm{O}>1$, corresponding in most cases to $\dot{M}<10^{-6} M_{\odot} \mathrm{yr}^{-1}$.

grains. As seen in Figures $9 a$ and 10, when (and whether) this ratio exceeds unity depends on competition between TDU and CBP in the intervals between dredge-up events. To look at this competition, we now examine the problem analytically.

The rate of addition of ${ }^{12} \mathrm{C}$ to the envelope by TDU is some function of time, $F(t)$. CBP removes material from the envelope and replaces it with material in which some of the ${ }^{12} \mathrm{C}$ has been destroyed. The time evolution of the envelope ${ }^{12} \mathrm{C}$ abundance is then governed by the equation

$$
\dot{Y}_{12}^{E}=\frac{\dot{M}}{M_{E}}\left(Y_{12}^{P}-Y_{12}^{E}\right)+M_{E}^{-1} F(t) .
$$

We wish to find the condition on $\dot{M}$ such that the envelope is carbon-rich at the end of the TP-AGB. If $Y_{12}^{P}=$ $(1-\alpha) Y_{12}^{E}$ (where $\alpha$ is the fraction of ${ }^{12} \mathrm{C}$ destroyed in a single pass), we then have

$$
\dot{Y}_{12}^{E}=-\frac{\dot{M}}{M_{E}} \alpha Y_{12}^{E}+\frac{F}{M_{E}} .
$$

The function $F(t)$ is essentially a sum of delta functions from dredge-up, but we approximate it by its mean value, $\langle F\rangle=12 \times 4.3 \times 10^{-6} Y_{12}^{E}(0) M_{E} \mathrm{yr}^{-1}$ (see Fig. $9 a$ ). The solution to equation (21) is

$$
Y_{12}^{E}(t)=e^{-\dot{M} \alpha t / M_{E}} Y_{12}^{E}(0)+\frac{\langle F\rangle}{\dot{M} \alpha}\left(1-e^{-\dot{M} \alpha t / M_{E}}\right) .
$$

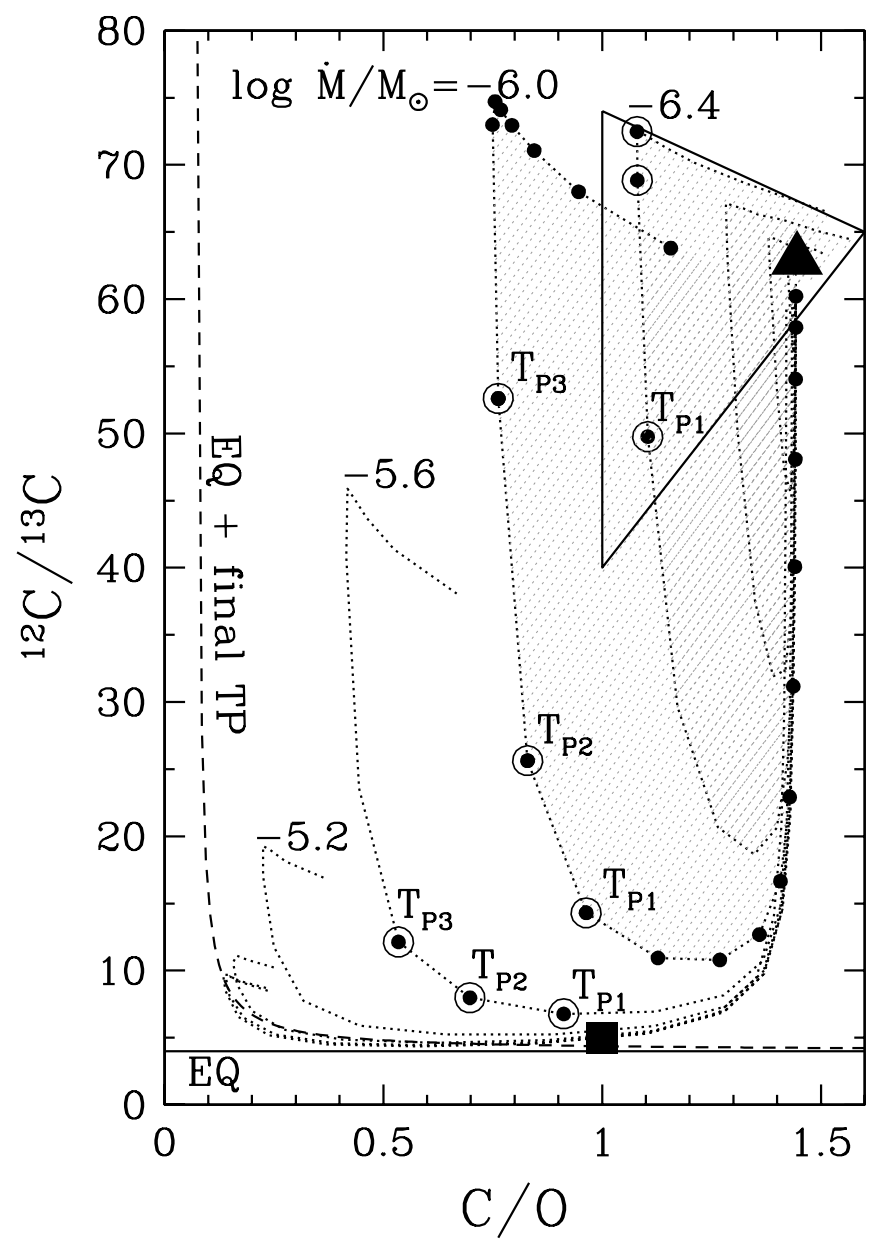

FIG. 11.-Final compositions of the envelope where CBP stops immediately before the last thermal pulse, after which the last dredge-up episode adds ${ }^{12} \mathrm{C}$. The $T_{P}$ increases clockwise along the constant- $\dot{M}$ curves; dots on the $\dot{M}=10^{-6} M_{\odot} \mathrm{yr}^{-1}$ curve indicate $\log T_{P}$ values in steps of 0.016 , and points with $\log T_{P 1}=7.616, \log T_{P 1}=7.632$, and $\log T_{P 3}=7.648$ are circled and labeled. The filled triangle indicates the final composition of a $1.5 M_{\odot}$ star without CBP. The shaded region contains $\dot{M}<10^{-6} M_{\odot} \mathrm{yr}^{-1}$, approximately the region of carbon star formation, while the large open triangle encloses compositions that have $\mathrm{C} / \mathrm{O}>1$ and ${ }^{26} \mathrm{Al} /{ }^{27} \mathrm{Al}>10^{-3}$. Note that low- ${ }^{12} \mathrm{C} /{ }^{13} \mathrm{C}$ carbon stars are restricted to a small region in the lower right-hand corner, near the filled square. EQ is the limiting equilibrium value. The bounding curve " $\mathrm{EQ}+$ final $\mathrm{TP}$ " corresponds to ${ }^{12} \mathrm{C}$ added by the final dredge-up episode, mixing with an envelope of composition EQ.

At the end of the TDU phase, $t=8.5 \times 10^{5} \mathrm{yr}$. The condition that the envelope is carbon-rich is that $Y_{12}^{E} / Y_{16}^{E}>1$. Inserting this condition along with $Y_{12}^{E}(0)=1.7 \times 10^{-4}$ into equation (22), dividing by $Y_{16}^{E}=5.9 \times 10^{-4}$, and solving for $\dot{M}$ gives

$$
\dot{M}<\frac{7 \times M_{E} \times 10^{-7} \mathrm{yr}^{-1}}{\alpha} .
$$

The value of the fraction destroyed $(\alpha)$ depends strongly on $T_{P}$ and weakly on $\dot{M}$. A reasonable mean value of $M_{E}$ during the TDU period is $0.4 M_{\odot}$. If $\alpha \sim 1$, then $\dot{M} \lesssim 3 \times 10^{-7}$ $M_{\odot} \mathrm{yr}^{-1}$ for $\mathrm{C} / \mathrm{O}>1$, within a factor of a few of what we find numerically. If $\dot{M}=10^{-4} M_{\odot} \mathrm{yr}^{-1}$, the maximum CBP rate that we considered, then $\mathrm{C} / \mathrm{O}>1$ in the envelope requires $\alpha \leq 3 \times 10^{-3}$, or very little carbon destruction per pass. The combination of high $\dot{M}$ and $\mathrm{C} / \mathrm{O}>1$ is therefore restricted to very low $T_{P}$. 
If the true efficiency of dredge-up is different from that for $\operatorname{SEM}(1.5)$, then $\langle F\rangle$ differs from the value used here (for a possible mechanism, see Herwig et al. 1997). If $\langle F\rangle$ is doubled, then the $\dot{M}$ that divides $\mathrm{C} / \mathrm{O}>1$ from $\mathrm{C} / \mathrm{O}<1$ (eq. [23]) increases by a factor of 3 ; we find that the relationship between the two parameters is approximately linear over this range of $\langle F\rangle$. Since dredge-up does not have a strong effect on the other abundances, the only influence on our results is to change which $\dot{M}$ lie on which side of $\mathrm{C} / \mathrm{O}<1$. The abundance changes found above for the $\mathrm{O}$ and below for the $\mathrm{N}$ isotopes take place over wider ranges in $\dot{M}$ than factors of 3, so we do not expect qualitative changes in our results if the dredged-up compositions change.

\subsection{Nitrogen}

The effect of CBP on nitrogen is to efficiently destroy ${ }^{15} \mathrm{~N}$ and to greatly increase ${ }^{14} \mathrm{~N}$ in the envelope. The increase in ${ }^{14} \mathrm{~N}$ is due to the conversion of ${ }^{12} \mathrm{C}$ into ${ }^{14} \mathrm{~N}$ by CBP at $\log T_{P} \gtrsim 7.55$. Complete conversion of ${ }^{12} \mathrm{C}$ in the envelope from the initial $\mathrm{C}$ inventory in the absence of TDU would increase the ${ }^{14} \mathrm{~N}$ abundance to over 3 times the original main-sequence value. Since TDU considerably increases the amount of available carbon in the envelope, even more ${ }^{14} \mathrm{~N}$ will be produced. This can, at the end of the stellar lifetime, give $1.5 \leq \mathrm{N} / \mathrm{O} \lesssim 8.6$ (with values above 1.7 involving destruction of oxygen). Such large amounts could constitute

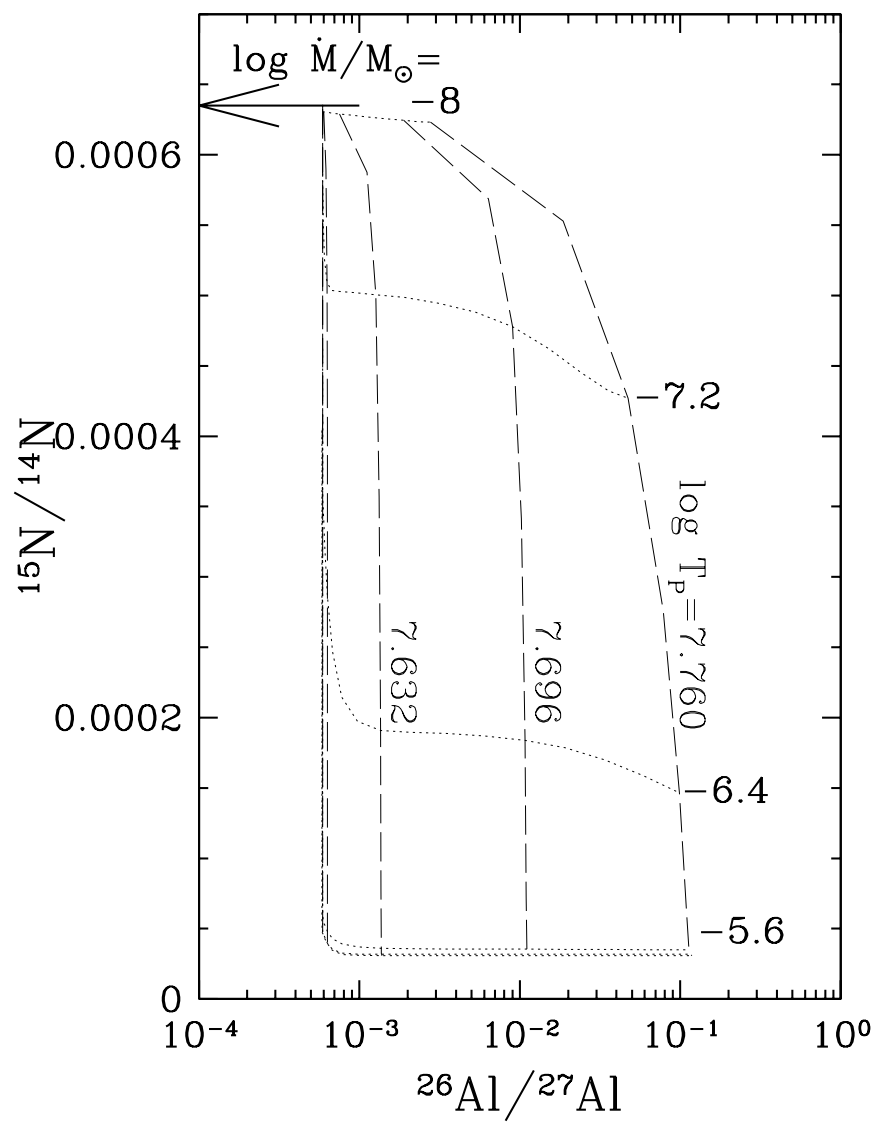

FIG. $12 a$ an important source of primary (scaling linearly with metallicity) nitrogen in the Galaxy.

The relationship between ${ }^{15} \mathrm{~N} /{ }^{14} \mathrm{~N}$ and ${ }^{26} \mathrm{Al} /{ }^{27} \mathrm{Al}$ is shown in Figure 12a for the final envelope composition as a function of $T_{P}$ and $\dot{M}$ for the numerical model. It is seen that there is a rapid drop in ${ }^{15} \mathrm{~N} /{ }^{14} \mathrm{~N}$ with increasing $\dot{M}$ for all $\log T_{P}>7.50$. For $\log T_{P}>7.62$, the ${ }^{15} \mathrm{~N} /{ }^{14} \mathrm{~N}$ ratio is essentially constant for a given $\dot{M}$. Comparing with Figure 10 , we see that a wide range in ${ }^{15} \mathrm{~N} /{ }^{14} \mathrm{~N}$ (from about $3 \times 10^{-5}$ to $6 \times 10^{-4}$ ) may occur if $\mathrm{C} / \mathrm{O}>1$. If $\mathrm{C} / \mathrm{O}<1$, then $\dot{M} \gtrsim 10^{-6.4} M_{\odot} \mathrm{yr}^{-1}$ and ${ }^{15} \mathrm{~N} /{ }^{14} \mathrm{~N} \lesssim 3 \times 10^{-4}$. See the discussion of $\mathrm{SiC}$ grains in $\S$ 4.1.2.

In Figure $12 b$ we show $\mathrm{N} / \mathrm{O}$ versus $\mathrm{C} / \mathrm{O}$ for the final composition of the envelope in the numerical model. The curves for different $\dot{M}$ and $T_{P}$ are indicated. It can be seen that CBP will produce high N/O for $\dot{M} \gtrsim 10^{-6.4} M_{\odot} \mathrm{yr}^{-1}$ and $\log T_{P} \gtrsim 7.6$. We note that $\mathrm{C} / \mathrm{O} \geq 1$ requires $\dot{M} \lesssim 10^{-6.4} M_{\odot}$ $\mathrm{yr}^{-1}$ for the same $T_{P}$.

\section{SELF-CONSISTENCY AND RELATION TO OTHER MODELS}

\subsection{Effects on Energy Generation}

We now examine the self-consistency of the postprocessing approach with the basic AGB stellar model. Since we assume an underlying stellar model $[\operatorname{SEM}(1.5)]$ without

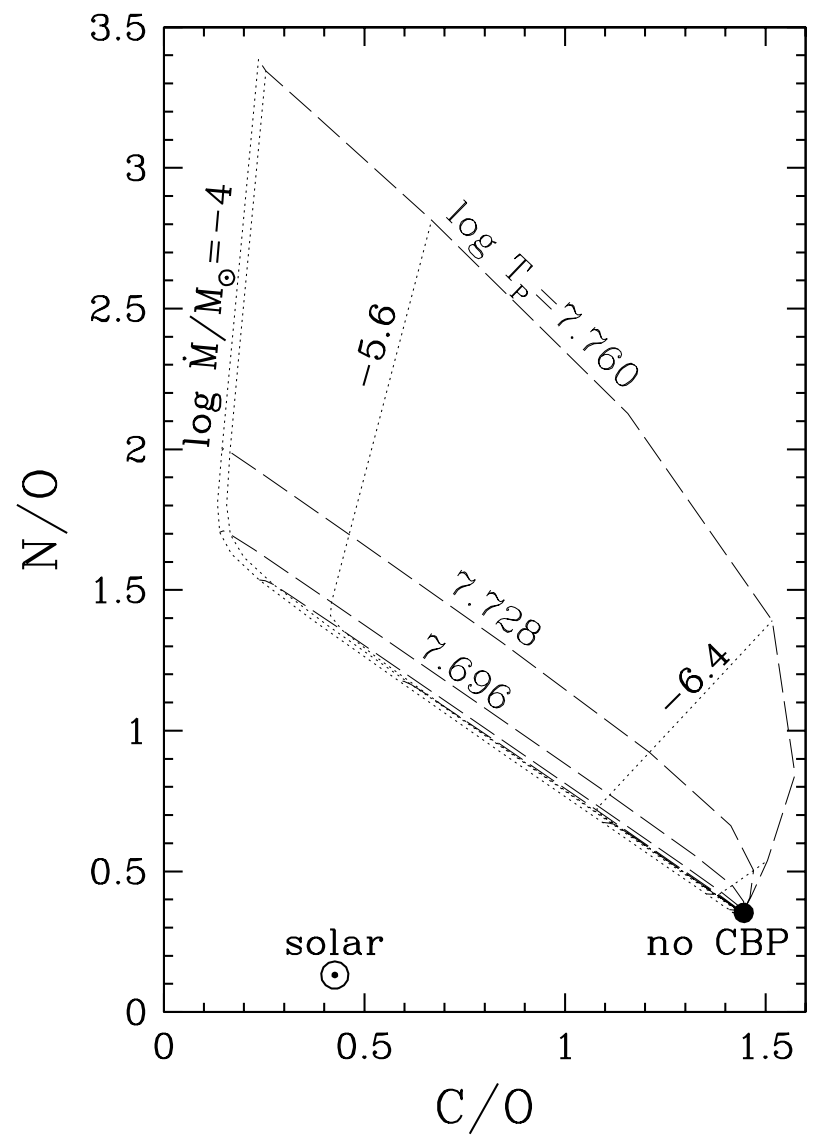

FIG. $12 b$

FIg. 12.-Final envelope compositions for the numerical model. (a) ${ }^{15} \mathrm{~N} /{ }^{14} \mathrm{~N}$ decreases from an initial value of $6.3 \times 10^{-6}$ toward a final value of $\sim 3 \times 10^{-5}$ for all $T_{P}$, but with an effectiveness that varies mainly with $\dot{M}$. The arrow at the top indicates the envelope composition after first dredge-up. $(b)$ Final values of $\mathrm{N} / \mathrm{O}$ and $\mathrm{C} / \mathrm{O}$. At most temperatures, this reflects varying degrees of conversion of $\mathrm{C}$ into $\mathrm{N}$; the two curves with the $\log T_{P}>7$ reflect additional destruction of O to make more C and N. The point labeled "solar" indicates solar system composition. 
CBP, it is important that the additional processing not affect the energy generation in a way that would significantly alter the evolution. The important issue is to determine what values of $T_{P}$ and $\dot{M}$ plausibly allow a self-consistent calculation.

For $\dot{M}$, we have considered the range $10^{-8}$ to $10^{-4} M_{\odot}$ 。 $\mathrm{yr}^{-1}$. The low end is comparable to the rate of advance of the $\mathrm{H}$ shell and too slow to turn over the radiative region between two successive thermal pulses. At the high end, the rate at which the radiative zone turns over corresponds to $\dot{M} / \Delta M \sim 0.1$ times $\mathrm{yr}^{-1}$. If the $\mathrm{CBP}$ circulation is to be distinct from envelope convection, its turnover rate should be at least an order of magnitude less than that of the convective envelope $\left(\sim 1 \mathrm{yr}^{-1}\right)$. Concerning the effective penetration depth $T_{P}$, we chose the lower limit of 7.44 for negligible processing and the upper limit of 7.76 to produce ${ }^{26} \mathrm{Al} /{ }^{27} \mathrm{Al} \sim 10^{-1}$. With this range in parameters, we now examine the effects on the energy production rate and luminosity. The energy generation in the parts of the stellar interior where $\mathrm{CBP}$ takes place occurs at very nearly the same rate with and without CBP. This is because CBP never changes the $\mathrm{H}$ mass fraction on a single pass through the radiative zone by more than $10^{-4}$ in the CBP models. It was calculated that $\mathrm{CBP}$ will change the $\mathrm{H}$ mass fraction in the envelope over the whole evolution by a total of only $4.6 \%$ even at the highest $T_{P}$ we considered. Since we have assumed that CBP changes neither the physical conditions in the radiative zone nor the total number of $\mathrm{C}$ and $\mathrm{N}$ catalyst nuclei, the energy generation rate can change by at most $4.6 \%$. An estimate of the energy generated in the matter undergoing $\mathrm{CBP}$ is computed as follows: We examine the $\mathrm{H}$ mass fraction of the envelope at the end of the numerical calculation and find the difference from the final mass fraction in the original SEM(1.5) model in the absence of CBP. We multiply this difference by an average envelope mass of $0.6 M_{\odot}$ and then by $7 \mathrm{MeV}$ nucleon ${ }^{-1}$, and divide by the $8.5 \times 10^{5}$ yr that CBP was active. This yields an effective luminosity, shown in Figure 13 as a function of $T_{P}$. For $\dot{M}>10^{-7} M_{\odot} \mathrm{yr}^{-1}$, this luminosity is independent of $\dot{M}$ (because, just as in the case of ${ }^{26} \mathrm{Al}$, only a small amount of processing occurs on each passage through the radiative zone). Also plotted in Figure 13 is the evolving luminosity of the $\operatorname{SEM}(1.5)$ model for comparison.

For $\log T_{P}=7.76$, the CBP flow yields at most $25 \%$ of the star's total energy generation rate during the TP-AGB phase. However, this amount is very close to the energy generation that would occur in the same radiative layers without CBP; the difference is $1.3 \%$ of the star's total energy output. Our proposal to explain ${ }^{26} \mathrm{Al} /{ }^{27} \mathrm{Al}$ ratios by $\mathrm{CBP}$ requires $\log T_{P}$ extending only to $\log T_{P} \sim 7.73$; this corresponds to $5 \%$ of the total energy generation occurring in the CBP flow and depletes about $1.2 \%$ of the envelope hydrogen. It follows that the $\mathrm{CBP}$ necessary to produce ${ }^{26} \mathrm{Al} /{ }^{27} \mathrm{Al} \sim 10^{-2}$ as found in some grains does not have a major effect on the luminosity or the energy generation rate.

\subsection{Extra Mixing by Diffusion Parameterization}

We now wish to compare models in which extra mixing is modeled as diffusive transport with those in which it is modeled as a mass flow (the present work; Boothroyd et al. 1994, 1995; WBS; Boothroyd \& Sackmann 1999; Sackmann \& Boothroyd 1999; Messenger 2000). The mass-flow models have only two parameters, $T_{P}$ and $\dot{M}$. In the case of diffusive

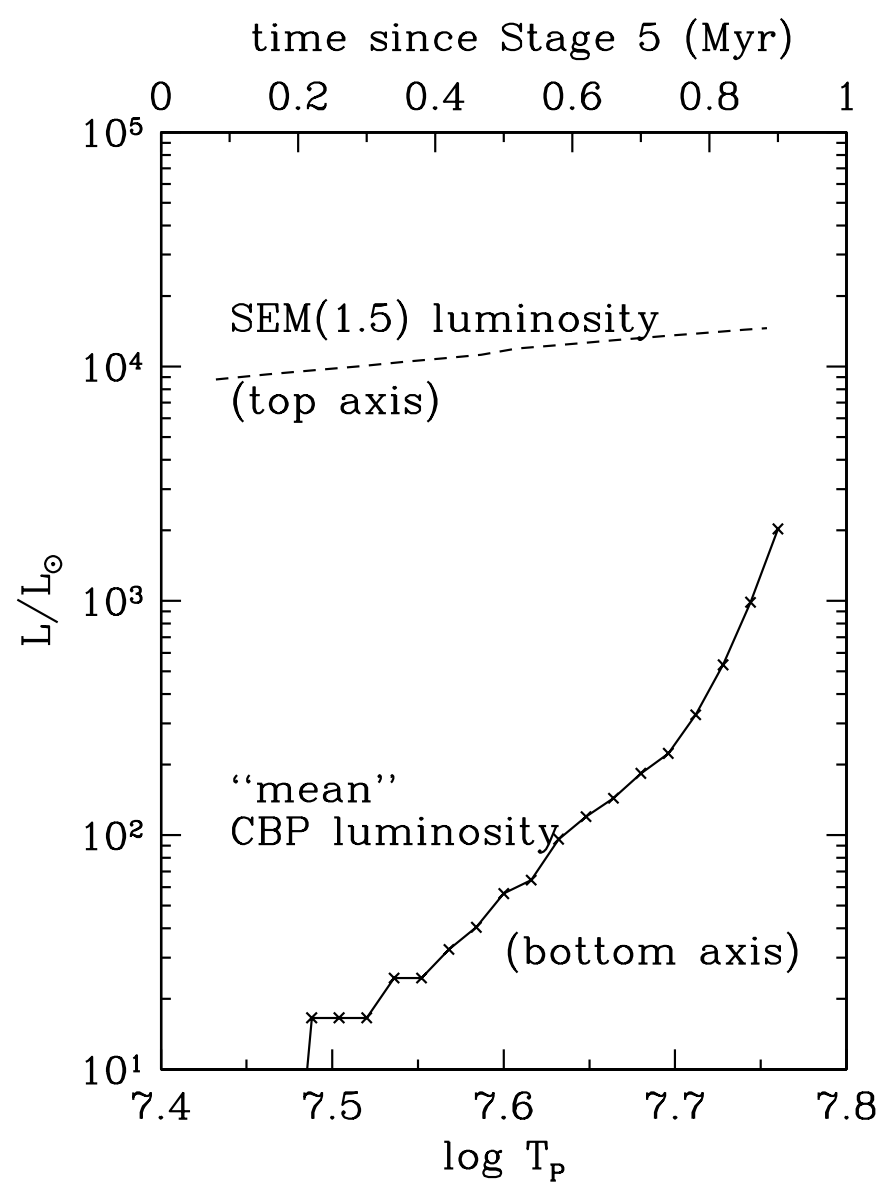

FIG. 13.-Solid curve: Mean luminosity in the CBP circulating regions as a function of $T_{P}$, estimated as described in the text (read bottom axis). Dashed curve: Luminosity of the $\operatorname{SEM}(1.5)$ model without CBP, as a function of time (read top axis).

models, the mixing is modeled either by postprocessing calculations, which include time evolution of the radiative zone, or by inclusion in a full stellar evolution code. In either case, a term is added to the stellar evolution model for diffusion between the convective envelope and a region of the radiative zone. The parameters used are a diffusion coefficient $D_{\text {mix }}$ and a maximum depth of diffusive mixing $\delta M_{\text {mix }}$. The parameter $\delta M_{\text {mix }}$ is defined in terms of the deepest mass coordinate penetrated $M_{\text {mix }}$, the convective-envelope boundary $M_{\mathrm{BCE}}$, and the base of the $\mathrm{H}$ shell $M_{\mathrm{H}}$ (see Fig. 1) as

$$
\delta M_{\text {mix }}=\frac{M_{\text {mix }}-M_{\mathrm{H}}}{M_{\mathrm{BCE}}-M_{\mathrm{H}}} .
$$

To obtain adequate nuclear processing, the diffusive penetration must extend into the upper part of the $\mathrm{H}$ shell. To prevent the catastrophe of interference with the region of maximum energy generation, a barrier boundary is assumed across which no diffusion is permitted to occur: this is specified as $\delta M_{\text {mix }}$ (see Denissenkov \& Weiss 1996; Denissenkov et al. 1998; Weiss et al. 2000). The two versions of transport-mass flow and diffusive mixing - are thus closely related and have similar effects. The relationships between $T_{P}$ and $\delta M_{\text {mix }}$ and between $\dot{M}$ and $D_{\text {mix }}$ can be seen as follows. The parameters $T_{P}$ and $\delta M_{\text {mix }}$ are directly related through the temperature at the position where the diffusive mixing is stopped. This is fixed by the basic stellar model. 
The relationship between $D_{\text {mix }}$ and $\dot{M}$ can be seen as follows. The length scale $l$ and timescale $t$ for mixing must satisfy $l^{2} / D t \sim 1$, so that $D_{\text {mix }} \approx l v$. For mass flow, the velocity $v$ corresponding to $\dot{M}$ at radial position $r$ in the star is $v=\dot{M} /\left[4 \pi r^{2} \rho(r)\right]$, where $\rho(r)$ is the mass density. This yields

$$
\begin{aligned}
D_{\text {mix }} \approx & \frac{l \dot{M}}{4 \pi r^{2} \rho} \\
\approx & 2 \times 10^{8} \mathrm{~cm}^{2} \mathrm{~s}^{-1} \frac{\dot{M}}{10^{-8} M_{\odot} \mathrm{yr}^{-1}} \\
& \times \frac{l}{3 \times 10^{10} \mathrm{~cm}}\left(\frac{\rho}{7 \mathrm{~g} \mathrm{~cm}^{-3}}\right)^{-1} .
\end{aligned}
$$

We have taken for reference the values of $r$ and $\rho$ at the $\mathrm{H}$ shell and $l$ the thickness of the radiative zone, all at stage 5 . This is the basic relationship between $D_{\text {mix }}$ and $\dot{M}$ to an order of magnitude. For comparison, the value estimated by Denissenkov \& Weiss (1996) is $D_{\text {mix }} \sim 10^{8} \mathrm{~cm}^{2} \mathrm{~s}^{-1}$ in their study of a low-metallicity RGB star. The relations between $\delta M_{\text {mix }}$ and $T_{P}$ for the evolutionary stages that we considered for CBP are shown in Figure 13. In the parametric diffusion treatments of Denissenkov and coauthors, $\delta M_{\text {mix }}$ was chosen to be where the $\mathrm{H}$ mass fraction was $5 \%$ $20 \%$ below the envelope value before the onset of mixing. We indicate the corresponding points in Figure 14. Their prescription results in mixing depths similar to those that we have examined in this paper.

\subsection{The Problem of ${ }^{26} \mathrm{Al}$ Production and $T_{P}$}

There remains the issue of whether the calculated effects of CBP for a star may be reasonably carried out by choosing a given $T_{P}$ and $\ddot{M}$ and using a selected radiative-zone structure from a single time (stage) during the TP-AGB. A complete calculation of $\mathrm{CBP}$ would require inclusion of the detailed evolution with time as the stellar structure evolves. Again let us consider a semianalytic treatment to understand the results. The temperature dependence of the processing is unimportant for carbon, nitrogen, and oxygen isotopes for all $T_{P} \gtrsim 7.60$. The results for these nuclides are thus almost independent of which stellar structure is chosen. We verified this by calculations with the numerical model, utilizing radiativezone structures from stages $3,4,5$, and 6 . This is not the case for ${ }^{26} \mathrm{Al}$, as can be seen by examining Figure $3 b$. For a given $T_{P}$, we see that the equilibrium ${ }^{26} \mathrm{Al} /{ }^{27} \mathrm{Al}$ for different times along the evolution (eq. [6]) decreases with increasing time. This rapid decrease occurs because as the $\mathrm{H}$ shell advances outward, the thickness (in mass coordinates) of the radiative zone decreases sharply, while the ratio of temperatures at the bottom and top of this region, $T_{\mathrm{H}} / T_{\mathrm{BCE}}$ (where $T_{\mathrm{BCE}}$ is the temperature at the bottom of the convective envelope), remains relatively fixed at about 24 . The region in which ${ }^{26} \mathrm{Al}$ is produced accordingly becomes thinner very rapidly as the star evolves, and the amount of ${ }^{26} \mathrm{Al}$ produced drops. Another contributing factor is that the density in the radiative zone drops steadily during the AGB phase, depressing all reaction rates. The final ${ }^{26} \mathrm{Al} /{ }^{27} \mathrm{Al}$ value calculated for a given $T_{P}$ is thus drastically altered by the choice of reference state used to provide the radiativezone conditions. That significant ${ }^{26} \mathrm{Al}$ production would occur is not an issue. Rather, the question is, how does

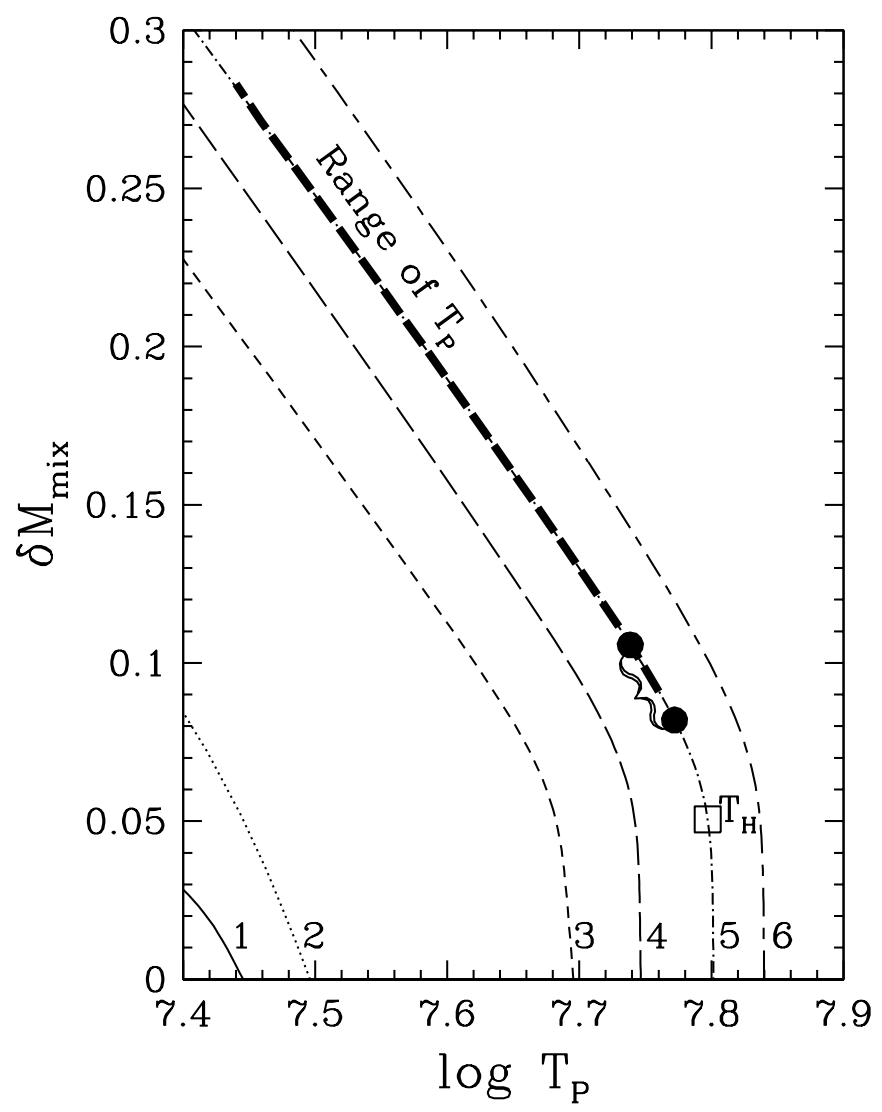

FIG. 14.-Relation between the parameters $\delta M_{\text {mix }}$ and $T_{P}$, at each of the six selected evolutionary stages of the radiative zone (see Table 1 and Fig. $3 b$ ). Along the stage 5 curve, the thick dashed line indicates $T_{P}$ considered in this study; the connected black dots indicate depths chosen to have $\mathrm{H}$ depleted by $5 \%-20 \%$, as assumed for $\delta M_{\text {mix }}$ in Weiss et al. (2000). This shows the essential equivalence of the mass-flow approach $\left(\dot{M}, T_{P}\right)$ and the diffusive mixing approach.

one pick an appropriate combination of stellar reference state and $T_{P}$ for ${ }^{26} \mathrm{Al}$ production? Figure 15 shows a graph of steady state ${ }^{26} \mathrm{Al} /{ }^{27} \mathrm{Al}$ ratios in the envelope as a function of $\log \left(T_{P} / T_{\mathrm{H}}\right)$ for the different stellar reference states considered. It can be seen that there is a congruent value of $T_{P}$ for each reference state that will produce essentially the same (within a factor of 3 ) ${ }^{26} \mathrm{Al} /{ }^{27} \mathrm{Al}$ if the value of $T_{P}$ is scaled with $T_{\mathrm{H}}$ as the star evolves. It follows that the $T_{P}$ required for ${ }^{26} \mathrm{Al}$ production is well constrained within this framework and that a CBP model covering all the isotopes of $\mathrm{C}, \mathrm{N}, \mathrm{O}, \mathrm{Mg}$, and $\mathrm{Al}$ can be reliably calculated for a given reference state. The particular value of $T_{P}$ is simply congruent to some effective value of $T_{P} / T_{\mathrm{H}}$ and is not otherwise fixed. This is in accord with the approach taken by Boothroyd \& Sackmann (1999) and Messenger (2000) for the RGB, but with the congruence made evident by ${ }^{26} \mathrm{Al}$ production rates.

\section{EXPERIMENTAL CONSEQUENCES}

We have constructed above a model that should be a good description of AGB stars undergoing CBP, using only two parameters. We now examine the consequences of the rules established by this model for observed isotopic abundances. 


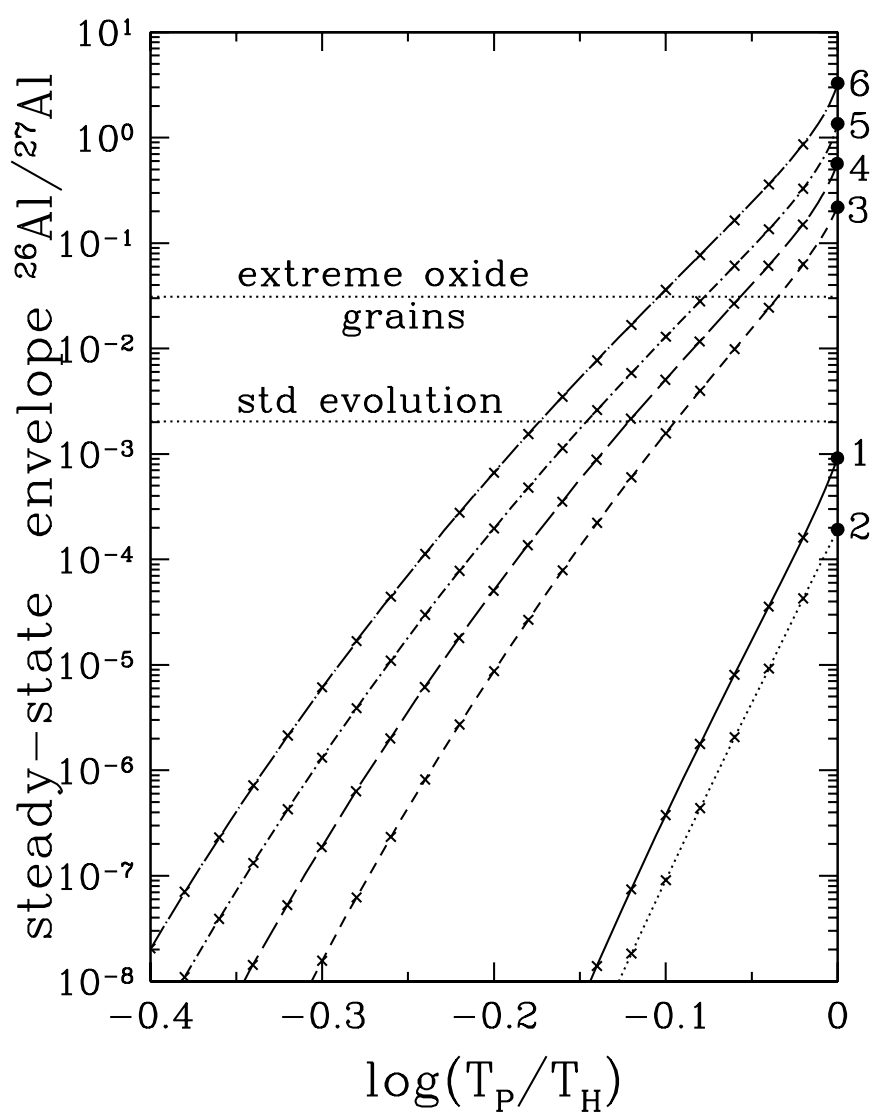

FIG. 15.- Steady state ${ }^{26} \mathrm{Al} /{ }^{27} \mathrm{Al}\left(\tau_{26} P / Y_{27}^{E}\right)$ calculated for the envelope assuming the whole evolution takes place with the radiative-zone structure fixed at stages 1-6, as labeled. The results are as in Fig. $3 b$, but in terms of $\log \left(T_{P} / T_{\mathrm{H}}\right)$. The ${ }^{26} \mathrm{Al}$ production is governed by the last stages. Note that for stages 4,5 , and 6 , abundant ${ }^{26} \mathrm{Al}$ is produced for $\log \left(T_{P} / T_{\mathrm{H}}\right)>-0.10$ and that $P \propto M_{E}^{-1}$.

\subsection{Circumstellar Grains}

\subsubsection{Regime of $\mathrm{C} / \mathrm{O}<1$}

Figure 16 exhibits the relationships between ${ }^{18} \mathrm{O} /{ }^{16} \mathrm{O}$ and ${ }^{17} \mathrm{O} /{ }^{16} \mathrm{O}$ in the envelope after first dredge-up (curve AF) and after CBP (thin dashed and dotted curves). The available data on oxide grains are also shown. A substantial population of the oxide grains can be seen to lie generally close to the curve AF for AGB evolution after first dredge-up for stars of varying mass, starting from initial solar isotopic composition. (Data are those summarized in Choi et al. 1998 and come from Hutcheon et al. 1994, Huss, Hutcheon, \& Wasserburg 1994, Nittler et al. 1994, 1997, 1998, and Nittler \& Cowsik 1997; curve AF is from Boothroyd \& Sackmann 1999). Some points lie above the curve AF and are plausibly explained by normal AGB evolution without CBP from initial states with elevated ${ }^{18} \mathrm{O} /{ }^{16} \mathrm{O}$ (Choi et al. 1998). The trajectories for $\mathrm{CBP}$ of ${ }^{18} \mathrm{O} /{ }^{16} \mathrm{O}$ and ${ }^{17} \mathrm{O} /{ }^{16} \mathrm{O}$ for a $1.5 M_{\odot}$ star of solar composition are given by the line from point $\mathrm{D}$ to point B, and the bounding curve for high $\dot{M}$ following Figure $7 a$. It is seen that there is a population of data that lie on this trend and a concentration of data approaching and near the equilibrium value at point B. Following the rules shown in Figure $7 b$, we see that data in the region labeled " $\mathrm{H}$ " may be explained by stars of up to $1.8 M_{\odot}$ initial mass. They may equally be explained by an initial state of somewhat lower ${ }^{18} \mathrm{O} /{ }^{16} \mathrm{O}$ without $\mathrm{CBP}$. Lower mass stars with solar

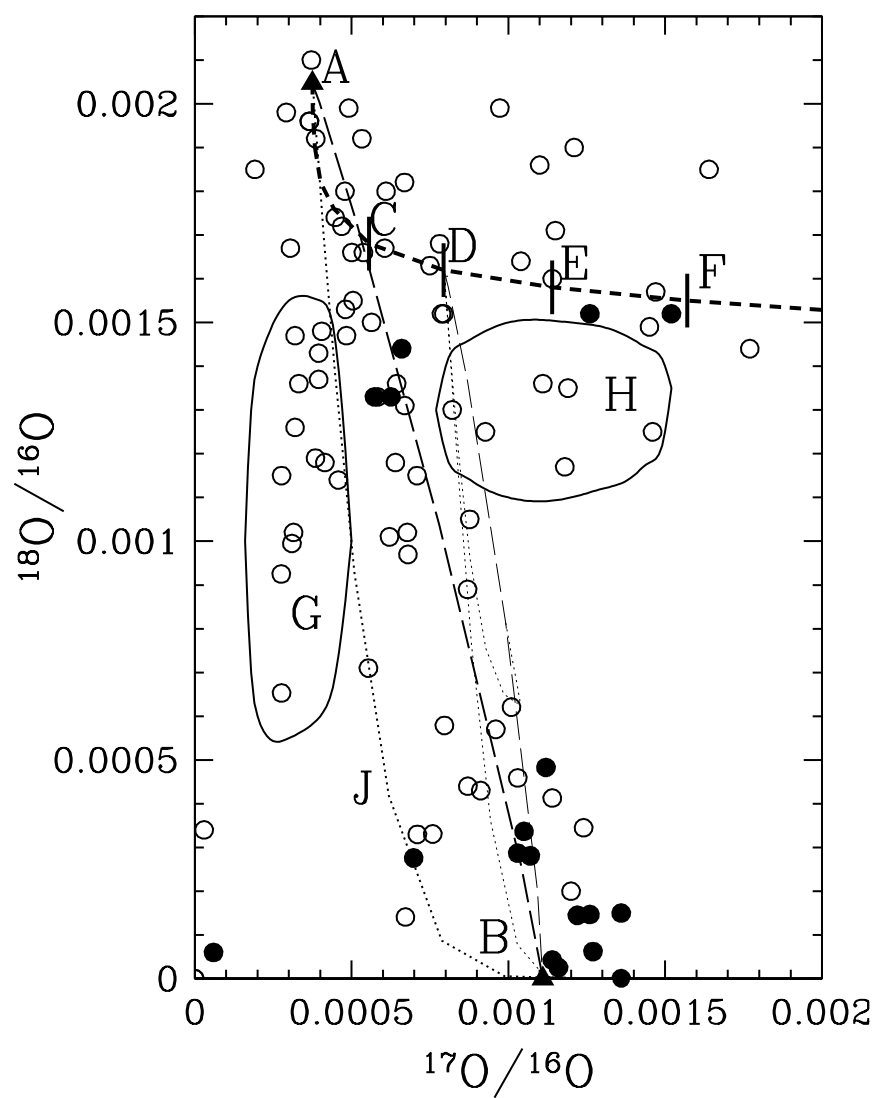

FIG. 16.-Final envelope ${ }^{18} \mathrm{O} /{ }^{16} \mathrm{O}$ and ${ }^{17} \mathrm{O} /{ }^{16} \mathrm{O}$ with oxide-grain data (summarized in Choi et al. 1998) superposed. The dotted curve AJB indicates the leftmost limit of compositions available starting from solar initial composition without dredge-up. Region $\mathrm{G}$ is therefore not accessible by CBP for a star with solar initial composition.

oxygen ratios directly modified by CBP would yield grains that lie in the region bounded to the left by the curve AJB and the line $\mathrm{AB}$, corresponding to a range in $\dot{M}$ and $T_{P}$. However, the data in region $G$ cannot be explained by AGB evolution from solar initial composition, with or without CBP; these must involve sources with very low to supersolar initial ${ }^{18} \mathrm{O} /{ }^{16} \mathrm{O}$ values and subsolar ${ }^{17} \mathrm{O} /{ }^{16} \mathrm{O}$ values, presumably representing different stages of Galactic evolution. The issue of how the abundances of the rare isotopes ${ }^{17} \mathrm{O}$ and ${ }^{18} \mathrm{O}$ evolve over time in local material and in an average over the Galaxy is complex (see Timmes \& Clayton 1996; Nittler \& Cowsik 1996; Alexander \& Nittler 1999). It is evident that the wide variety of oxygen isotopic abundances found in circumstellar oxides preserved in meteorites requires a diverse set of stellar sources with quite different initial isotopic abundances and metallicities. Inspection of Figure 10 shows that for $\mathrm{C} / \mathrm{O}<1$ in the stellar envelopes, there is a wide range of $T_{P}$ that produces the oxygen compositions shown in Figure 16, allowing a plausible explanation of a wide range of ${ }^{26} \mathrm{Al} /{ }^{27} \mathrm{Al}$ abundances. The production of ${ }^{26} \mathrm{Al}$ is quite adequate to obtain values of ${ }^{26} \mathrm{Al} /{ }^{27} \mathrm{Al}$ up to $\sim 6 \times 10^{-2}$, the governing parameter being $7.68 \lesssim \log T_{P} \lesssim 7.73$. The values of ${ }^{26} \mathrm{Al} /{ }^{27} \mathrm{Al} \lesssim 10^{-3}$ cannot be achieved within the framework of standard AGB evolution (BGW99) unless there are cases in which essentially negligible ${ }^{26} \mathrm{Al}$ production in the $\mathrm{H}$ shell is possible and the stellar lifetime is still short enough to contribute to the solar system grains. 


\subsubsection{Regime of $\mathrm{C} / \mathrm{O}>1$}

The regime where carbon or carbide grains may form under equilibrium conditions requires $\mathrm{C} / \mathrm{O}>1$. There are abundant data on ${ }^{12} \mathrm{C} /{ }^{13} \mathrm{C}$ and ${ }^{26} \mathrm{Al} /{ }^{27} \mathrm{Al}$ in circumstellar carbides recovered from meteorites (e.g., Amari, Lewis, \& Anders 1994; Hoppe et al. 1994; Hoppe \& Ott 1997; Huss et al. 1997). Most recently, the results have been summarized and discussed by Amari et al. (2001b, 2001c). In addition to the ${ }^{12} \mathrm{C} /{ }^{13} \mathrm{C}$ and ${ }^{26} \mathrm{Al} /{ }^{27} \mathrm{Al}$ data, there are results on ${ }^{14} \mathrm{~N} /{ }^{15} \mathrm{~N}$ as well as on $\mathrm{Ti}$ and $\mathrm{Si}$ isotopes. In the following discussion, we focus on the so-called mainstream (MS) grains, $\mathrm{A}$ and $\mathrm{B}$ grains, and $\mathrm{Y}$ grains. These classifications are dominantly based on the ratios ${ }^{12} \mathrm{C} /{ }^{13} \mathrm{C}$ and ${ }^{14} \mathrm{~N} /{ }^{15} \mathrm{~N}$ (see Amari et al. 2001c for more extensive discussion). The MS grains are overwhelmingly the most abundant of the circumstellar $\mathrm{SiC}$ grains found. Figure $17 a$ shows the distribution of grains in the ${ }^{12} \mathrm{C} /{ }^{13} \mathrm{C},{ }^{14} \mathrm{~N} /{ }^{15} \mathrm{~N}$ diagram. We first consider the MS grains. As noted by many previous workers, the ${ }^{12} \mathrm{C} /{ }^{13} \mathrm{C}$ of MS grains lies in the region expected for the envelopes of AGB stars of approximately solar metallicity. The spread of ${ }^{12} \mathrm{C} /{ }^{13} \mathrm{C}$ extends from slightly above the solar value of 89 down to 20 , with most grains between 40 and 80 . We also show the whole region (labeled " $\mathrm{P}$ " and " $Q$ ") that is accessible by CBP in the numerical model with $\mathrm{C} / \mathrm{O}>1$.

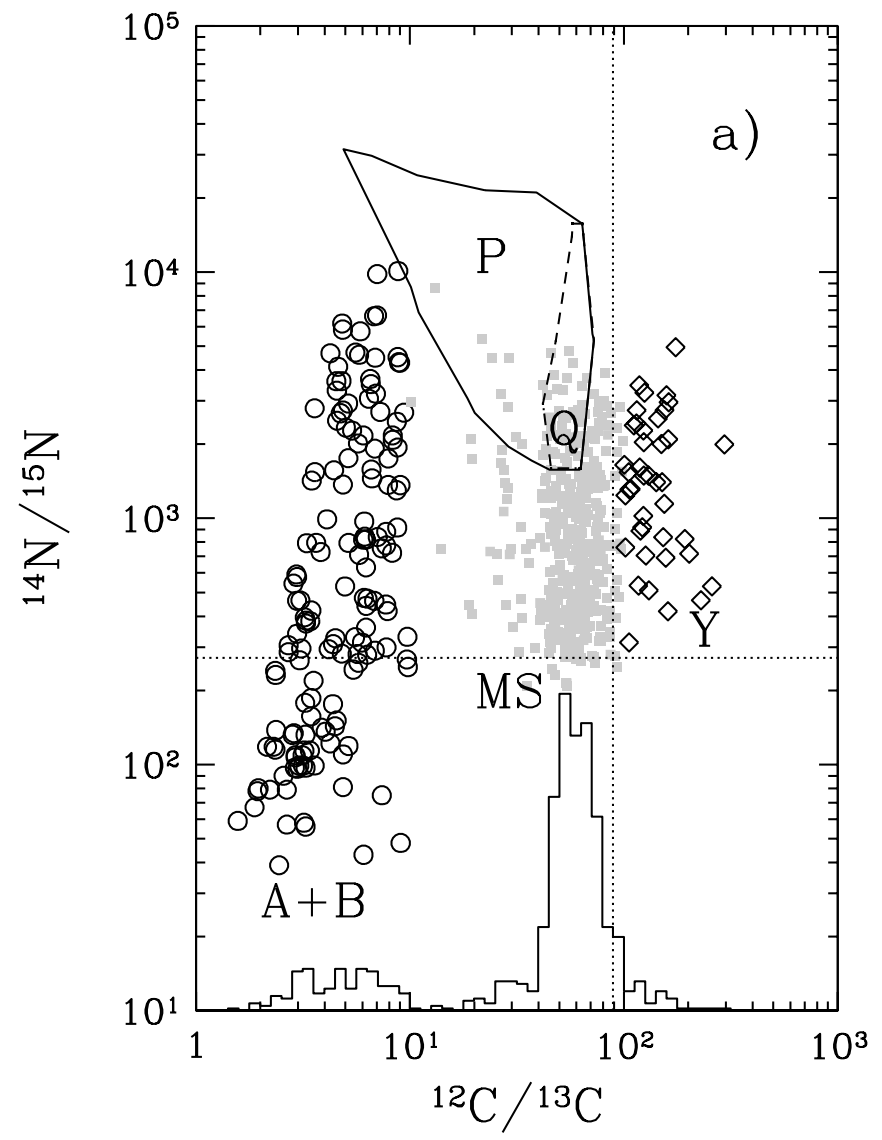

The observed ${ }^{26} \mathrm{Al} /{ }^{27} \mathrm{Al}$ versus ${ }^{12} \mathrm{C} /{ }^{13} \mathrm{C}$ is shown in Figure 18. The horizontal segment $\mathrm{P}$ with low ${ }^{12} \mathrm{C} /{ }^{13} \mathrm{C}$ corresponds to the high- ${ }^{14} \mathrm{~N} /{ }^{15} \mathrm{~N}$ region, also labeled " $\mathrm{P}$ " in Figure 17a. This is also the region in Figure 11 of low $T_{P}$ and $\mathrm{C} / \mathrm{O}>1$, running from the solid triangle (no $\mathrm{CBP}$ ) down to ${ }^{12} \mathrm{C} /{ }^{13} \mathrm{C} \sim 5$ at $\dot{M}>10^{-5.6} M_{\odot} \mathrm{yr}^{-1}$. There is no ${ }^{26} \mathrm{Al}$ produced since $T_{P}$ is low along this segment of the curve. The value of ${ }^{26} \mathrm{Al} /{ }^{27} \mathrm{Al} \sim 8 \times 10^{-4}$ in region $\mathrm{P}$ of Figure 18 corresponds to the total ${ }^{26} \mathrm{Al}$ produced in the conventional treatment of TDU (BGW99; see Fig. 3a). If we include an earlier episode of CBP (prior to TDU) sufficient to give ${ }^{26} \mathrm{Al} /{ }^{27} \mathrm{Al} \sim 5 \times 10^{-3}$, then the envelope that is accessible for $\mathrm{C} / \mathrm{O}>1$ is region $\mathrm{R}$ of Figure 18. The observed ${ }^{26} \mathrm{Al} /{ }^{27} \mathrm{Al}$ for the range of ${ }^{12} \mathrm{C} /{ }^{13} \mathrm{C}$ found in the MS grains is well represented by CBP. However, the samples with ${ }^{26} \mathrm{Al} /{ }^{27} \mathrm{Al}<6 \times 10^{-4}$ cannot be explained by the model (independent of $\mathrm{C} / \mathrm{O}$ ). A substantial fraction of these grains (and of oxide grains) thus have less ${ }^{26} \mathrm{Al}$ than predicted for conventional AGB evolution in stars of $\sim 1 M_{\odot}$ (BGW99; Mowlavi \& Meynet 2000). It is possible that this reflects the normal AGB evolution at somewhat lower stellar masses or that there is some variability in the non-CBP ${ }^{26} \mathrm{Al}$ production in AGB stars, as discussed in $\S 1$.

A fundamental problem with the $\mathrm{SiC}$ grains is exhibited by the ${ }^{14} \mathrm{~N} /{ }^{15} \mathrm{~N}$ data shown in Figure $17 a$. Here the majority of MS grains lie below ${ }^{14} \mathrm{~N} /{ }^{15} \mathrm{~N}=10^{3}$, and the lower values

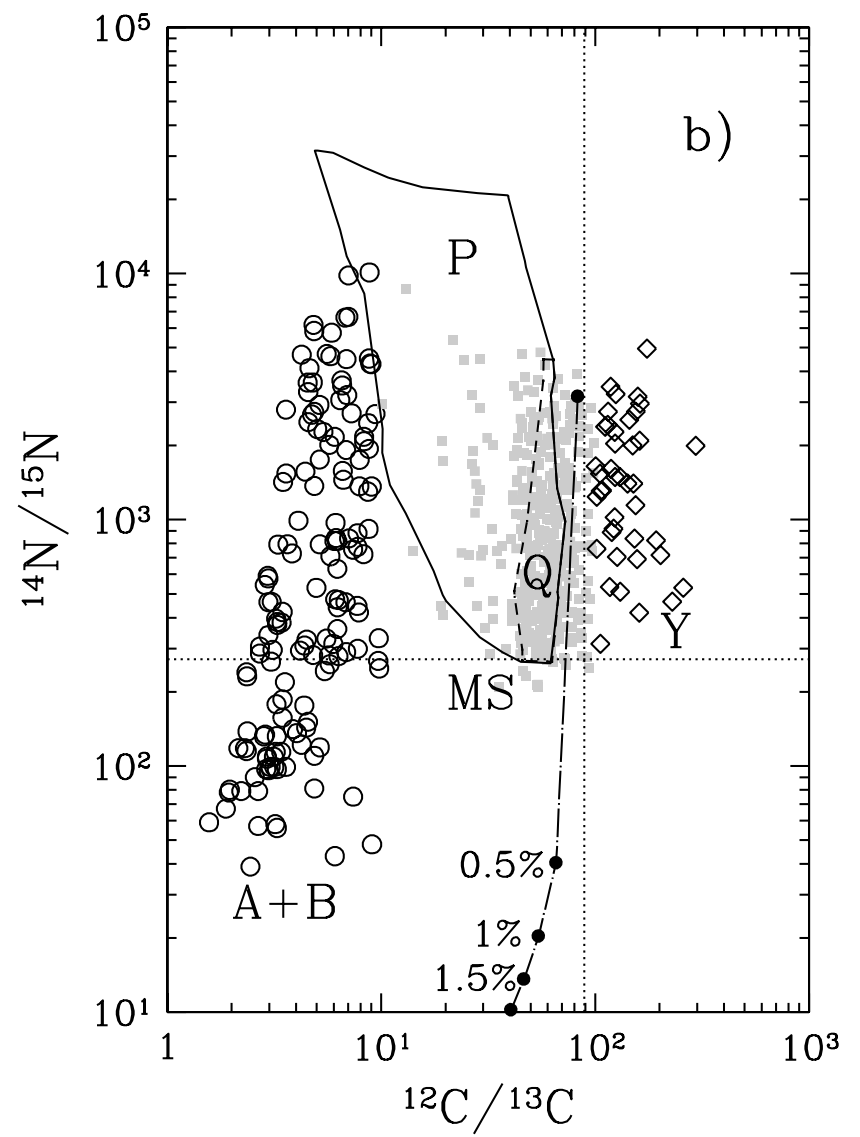

FIG. 17. $-{ }^{12} \mathrm{C} /{ }^{13} \mathrm{C}$ and ${ }^{14} \mathrm{~N} /{ }^{15} \mathrm{~N}$ of $\mathrm{SiC}$ grains compiled by Amari et al. (2001c). Symbols indicate $\mathrm{A}+\mathrm{B}$ grains (circles), MS grains (squares), and Y grains (diamonds). The histogram at the bottom is the frequency of each type (arbitrary linear units). (a) Regions $\mathrm{P}$ and $\mathrm{Q}$ contain all compositions with $\mathrm{C} / \mathrm{O}>1$ produced during TDU and CBP; Q is with ${ }^{26} \mathrm{Al} /{ }^{27} \mathrm{Al}>10^{-3}$. (b) Same model as in $(a)$, but for ${ }^{14} \mathrm{~N} /{ }^{15} \mathrm{~N}=270$ when the star arrived on the AGB, corresponding to an initial ${ }^{14} \mathrm{~N} /{ }^{15} \mathrm{~N} \sim 70$ when the star formed. The dot-dashed curve shows compositions that result when a typical low- $-{ }^{15} \mathrm{~N} /{ }^{14} \mathrm{~N}$ composition from an AGB star mixes with ejecta from a nova outburst. Points along the curve indicate the fraction of the composition made up of nova ejecta (nova composition from Starrfield et al. 2001). 


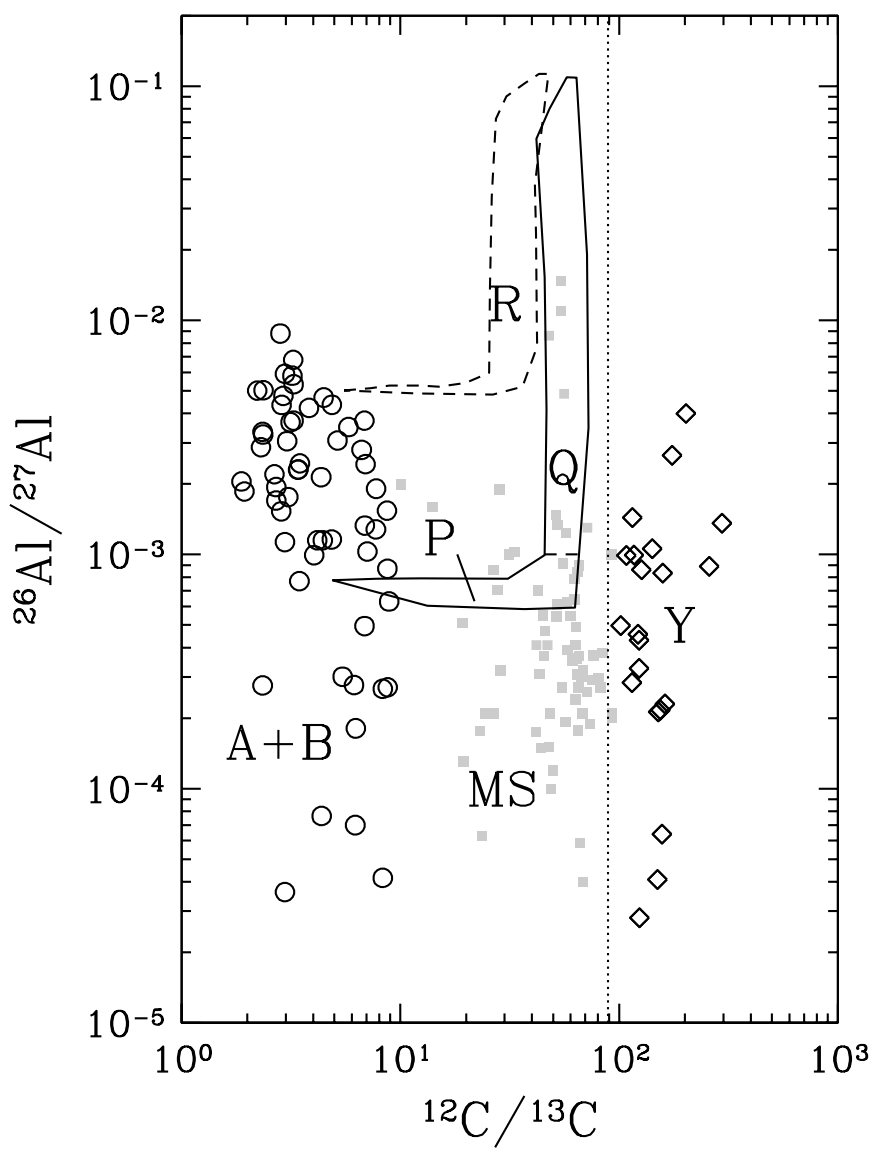

FIG. 18. $-\mathrm{C}$ and $\mathrm{Al}$ isotopic compositions of $\mathrm{SiC}$ grains compiled in Amari et al. (2001c; symbols as in Fig. 17). The region P-Q contains all compositions produced with TDU and CBP. For all values of $T_{P}$ and $\dot{M}$ considered (except for the small part of region $\mathrm{P}$ with ${ }^{12} \mathrm{C} /{ }^{13} \mathrm{C} \gtrsim 50$ ), these are the same regions as in Fig. 17. Region R is analogous to P-Q, but for models that assume that extensive $\mathrm{CBP}$ produced ${ }^{26} \mathrm{Al} /{ }^{27} \mathrm{Al}=10^{-2}$ and ${ }^{12} \mathrm{C} /{ }^{13} \mathrm{C}=3$ before TDU and then continued throughout TDU.

are near the solar value. This is exceedingly difficult to explain with AGB stars in any evolution model that assumes solar abundances as the initial state. First dredge-up will destroy roughly half of the ${ }^{15} \mathrm{~N}$ in the envelope and double the ${ }^{14} \mathrm{~N}$, so that the resulting value of ${ }^{14} \mathrm{~N} /{ }^{15} \mathrm{~N}$ should be $\sim 10^{3}$. Then any CBP will only destroy more ${ }^{15} \mathrm{~N}$ and produce more ${ }^{14} \mathrm{~N}$, increasing the ratio. To explain the high ${ }^{15} \mathrm{~N}$, it has been proposed by Huss et al. (1997) that the ${ }^{18} \mathrm{O}(p, \alpha){ }^{15} \mathrm{~N}$ reaction rates are far greater than the accepted values. If this is the case, then all of the ${ }^{15} \mathrm{~N}$ calculations presented here are in error. The nuclear data suffer from poorly known parameters for a low-energy resonance, but the most recent work (Champagne \& Pitt 1986) contradicts earlier indications of an additional large subthreshold resonance that would produce the much higher rate advocated by Huss et al. (1997). If we assume that the calculations presented here are correct and that the MS grains represent RGB and AGB evolution, then it is necessary to provide an alternative hypothesis. If the MS grains with ${ }^{14} \mathrm{~N} /{ }^{15} \mathrm{~N}<10^{3}$ were the products of evolution on the RGB prior to first dredge-up, then to produce grains with no change from the initial $\mathrm{N}$ composition would require $\mathrm{RGB}$ stars that have $\mathrm{C} / \mathrm{O}>1$ and that have done nothing to their $\mathrm{N}$ isotopes. No evidence for such stars, either theoretical or observational, has been found. If we consider that the stars that produced the grains with low ${ }^{14} \mathrm{~N} /{ }^{15} \mathrm{~N}$ were the products of normal first dredgeup and subsequent AGB evolution, then it is necessary that the stars' initial composition have ${ }^{14} \mathrm{~N} /{ }^{15} \mathrm{~N}$ far less than the solar value. In all cases, first dredge-up increases ${ }^{14} \mathrm{~N} /{ }^{15} \mathrm{~N}$ by a factor of $\sim 3.7$. If we assume that the typical initial state had ${ }^{14} \mathrm{~N} /{ }^{15} \mathrm{~N} \sim 70$, then first dredge-up would give the solar value, and subsequent CBP would essentially fill the region of the MS grains.

Following these considerations, we carried out calculations in which we increased the assumed initial ${ }^{15} \mathrm{~N}$ inventory so that ${ }^{14} \mathrm{~N} /{ }^{15} \mathrm{~N}=270$ (the solar value) when the star arrives on the AGB. We obtained results for all the pertinent isotopes with this initial composition in the numerical model. The results for $\mathrm{O}, \mathrm{C}$, and $\mathrm{Al}$ are of course not affected. The results for ${ }^{14} \mathrm{~N} /{ }^{15} \mathrm{~N}$ and ${ }^{12} \mathrm{C} /{ }^{13} \mathrm{C}$ are shown in Figure $17 b$. It can be seen that the whole mainstream region (not just the upper part) is covered with this shift in initial ${ }^{14} \mathrm{~N} /{ }^{15} \mathrm{~N}$. We note that adjusting the initial ${ }^{14} \mathrm{~N} /{ }^{15} \mathrm{~N}$ by decreasing the amount of ${ }^{14} \mathrm{~N}$ while holding all else fixed cannot provide the same adjustment of the final ${ }^{14} \mathrm{~N} /{ }^{15} \mathrm{~N}$. This is because first dredge-up with initially solar abundance ratios among the metals doubles the ${ }^{14} \mathrm{~N}$ mass fraction in the envelope. Adjustments to the initial ${ }^{14} \mathrm{~N}$ inventory do not affect the ${ }^{14} \mathrm{~N}$ gained at first dredge-up, so they can reduce ${ }^{14} \mathrm{~N} /{ }^{15} \mathrm{~N}$ on the AGB by no more than a factor of 2 below the standard models. The $\mathrm{SiC}$ grains require a reduction by almost a factor of 4 .

It is not clear that the solar ${ }^{14} \mathrm{~N} /{ }^{15} \mathrm{~N}$ value is in any way the proper choice for the ISM sampled in forming the stars that are the source of the MS grains. The data on oxide grains clearly demonstrate that the stellar sources must come from different molecular clouds that represent a wide range in degree of chemical evolution. The requirement that the bulk solar values represent a mix of diverse sources is found in many examples. This is particularly the case for rare isotopes such as ${ }^{13} \mathrm{C},{ }^{15} \mathrm{~N},{ }^{17} \mathrm{O}$, and ${ }^{18} \mathrm{O}$. We recall that ${ }^{17} \mathrm{O} /{ }^{18} \mathrm{O}$ in the ISM (Penzias 1981) has been found to be distinctly different from the solar value (a factor of $1.7 \pm 0.1$ ). We consider that there has been evolution of these isotopic abundances over Galactic history, that the abundances of some isotopes are quite different today than at the time the solar nebula formed, and that the abundances, particularly of very rare or low-abundance isotopes, will be distinctly different in different places, even at the same time. As is the case for oxygen, the ${ }^{14} \mathrm{~N} /{ }^{15} \mathrm{~N}$ values in the MS grains are clear indications that the initial values for some sources cannot be the solar value but must be greatly enhanced in ${ }^{15} \mathrm{~N}$. This conclusion is unavoidable if the grains are produced by AGB stars.

Many grains assigned to the MS group exhibit clear $s$ process signatures in several heavy elements, which are attributed to nucleosynthesis in AGB stars, as noted by Hoppe \& Ott (1997) and Gallino, Busso, \& Lugaro (1997). The more abundant isotopic data on $\mathrm{Si}$ and $\mathrm{Ti}$ in $\mathrm{SiC}$ grains show that these elements are dominated by effects associated with Galactic evolution (Gallino et al. 1994), with small modifications attributed to $s$-processing. The $\mathrm{Si}$ and $\mathrm{Ti}$ data are not useful for our arguments because they do not strongly reflect the $s$-process. However, the important new generation of measurements on individual mainstream $\mathrm{SiC}$ grains for many elements show clear $s$-process signatures in several isotopes of different elements (Nicolussi et al. 1997, 1998; Pellin et al. 2000). In summary, there are compelling reasons to assign MS grains to AGB stars, as recognized by 
many workers. The largest problem with this assignment appears to be the ${ }^{14} \mathrm{~N} /{ }^{15} \mathrm{~N}$ data. We conclude that this can most simply be resolved by assuming that the initial ${ }^{14} \mathrm{~N} /{ }^{15} \mathrm{~N}$ typically available in molecular clouds $\sim 4.6 \times 10^{9}$ yr ago must have ranged from ${ }^{14} \mathrm{~N} /{ }^{15} \mathrm{~N} \sim 70$ upward. The "solar" value is considered to be the result of a sampling of material that had previously undergone some prior processing in AGB stars.

The $\mathrm{N}$ isotopic compositions determined for the ISM seem to support the idea that ${ }^{14} \mathrm{~N} /{ }^{15} \mathrm{~N}$ increases from low values in regions of low astration to higher values in regions of high astration. Chin et al. 1999 find ${ }^{14} \mathrm{~N} /{ }^{15} \mathrm{~N} \sim 100$ for the Large Magellanic Cloud, while higher values of 270 in the solar system, 200-600 in the Galactic disk, and greater than 600 at the Galactic center are found in places with presumably increasing astration (see Wilson \& Rood 1994 and references therein). It is thus plausible that ${ }^{14} \mathrm{~N} /{ }^{15} \mathrm{~N}$ was typically much lower at earlier times and certain that it is variable. On the other hand, it is generally claimed (e.g., Wilson $\&$ Rood 1994) that there is a gradient of ${ }^{14} \mathrm{~N} /{ }^{15} \mathrm{~N}$ with Galactocentric distance in the Galactic disk, in a direction suggesting that ${ }^{14} \mathrm{~N} /{ }^{15} \mathrm{~N}$ decreases with astration.

Inspection of Figures $17 a, 17 b$, and 18 shows the data on $\mathrm{Y}$ grains, which are classified as distinctive almost solely because their ${ }^{12} \mathrm{C} /{ }^{13} \mathrm{C}$ is greater than the solar value. The distributions of these grains in both ${ }^{14} \mathrm{~N} /{ }^{15} \mathrm{~N}$ versus ${ }^{12} \mathrm{C} /{ }^{13} \mathrm{C}$ and ${ }^{26} \mathrm{Al} /{ }^{27} \mathrm{Al}$ versus ${ }^{12} \mathrm{C} /{ }^{13} \mathrm{C}$ are otherwise the same as those for the MS grains. If one considers ${ }^{12} \mathrm{C}$ dredge-up in models of AGB stars, it is evident that an increase in the amount of dredge-up by a factor of 2 or 3 for a small proportion of AGB stars would yield a carbon star with Y-grain-like composition. In this case, the Y grains are not distinguishable from the more general population of grains produced by AGB stars but reflect some variation in $\mathrm{C}$ dredge-up. Our preferred interpretation is that they simply reflect the high-end tail of the $\mathrm{C} / \mathrm{O}$ distribution resulting from dredge-up, which may or may not result from variation of metallicity (i.e., variation of initial O). The extent to which the variability of ${ }^{12} \mathrm{C}$ dredge-up is possible within the framework of AGB models remains to be tested. Other proposals to explain the $\mathrm{Y}$ grains are based on the dependence of dredge-up on metallicity, as argued by, e.g., Amari et al. (2001c) and references therein on the basis of anomalies in the $\mathrm{Si}$ isotopic composition.

We now consider the A and B grains. As discussed by Amari et al. (2001c), these grains are defined by low ${ }^{12} \mathrm{C} /{ }^{13} \mathrm{C}<10$. They are also found to exhibit ${ }^{14} \mathrm{~N} /{ }^{15} \mathrm{~N}$ ranging over more than 2 orders of magnitude and extending to below the solar value. We consider them here as representing a group of grains produced by a generic process. It is evident that AGB stars can produce ${ }^{12} \mathrm{C} /{ }^{13} \mathrm{C}$ as low as 4 at low $T_{P}$, but they cannot produce a wide range in ${ }^{14} \mathrm{~N} /{ }^{15} \mathrm{~N}$ and ${ }^{26} \mathrm{Al} /{ }^{27} \mathrm{Al}$ and simultaneously have ${ }^{12} \mathrm{C} /{ }^{13} \mathrm{C} \lesssim 12$ and $\mathrm{C} / \mathrm{O}>1$. It follows that an AGB source at approximately solar metallicity cannot be the origin of such grains. Further, we do not consider that this condition can be directly related to low metallicity. It should also be noted that a considerable number of these grains have ${ }^{12} \mathrm{C} /{ }^{13} \mathrm{C}<3$, ranging down to ${ }^{12} \mathrm{C} /{ }^{13} \mathrm{C}=1.57 \pm 0.06$ and $1.8 \pm 0.01$ (Amari et al. 2001c). From the existing knowledge of reaction rates, values below 3 cannot be achieved on either the RGB or the AGB with or without CBP. It follows that either there is some significant $(\times 2)$ error in the reaction rates or these grains were produced under conditions quite different than the $\mathrm{CNO}$ burning outlined in $\S 2.3$ and as discussed by other workers. A considerable number of the grains have ${ }^{14} \mathrm{~N} /{ }^{15} \mathrm{~N}$ extending down to ${ }^{14} \mathrm{~N} /{ }^{15} \mathrm{~N}=40$, far below the solar value. It was suggested by Amari et al. (2001c) that this may be produced by hot $\mathrm{H}$ burning (far above AGB shell-burning temperatures), possibly associated with novae. While it is reported that many $\mathrm{CO}$ novae condense carbon dust, it should be noted that only three of the 31 recent novae listed by Gehrz et al. (1998) have been observed to have C/O $>1$. The formation of different types of grains (e.g., carbides or silicates) in the same nova may reflect sequential condensation (see Gehrz et al. 1998). The calculated yields for thermonuclear runaways (TNRs) in novae almost exclusively have $\mathrm{C} / \mathrm{O}<1$ (Starrfield et al. 2001; S. Starrfield 2001, private communication). The most recent calculations (S. Starrfield 2001, private communication; S. Starrfield et al. 2003, in preparation) show that $\mathrm{C} / \mathrm{O}<1$ for white dwarf masses of 1.25 and $1.35 M_{\odot}$. An extremely high ${ }^{17} \mathrm{O} /{ }^{16} \mathrm{O}$ ratio $(>1)$ is predicted in all cases. A search for implanted oxygen with such extreme ${ }^{17} \mathrm{O}$ enrichments in graphite or $\mathrm{SiC}$ grains would therefore be very important. It is known that extreme values of ${ }^{12} \mathrm{C} /{ }^{13} \mathrm{C}$ and ${ }^{14} \mathrm{~N} /{ }^{15} \mathrm{~N}(\lesssim 1.5)$, as well as substantial ${ }^{26} \mathrm{Al}$, are predicted for novae yields (see Starrfield et al. 2001). Nonetheless, it is difficult to connect the A and $\mathrm{B}$ grains as a group to novae sources. There are only two reported cases of high ${ }^{26} \mathrm{Al} /{ }^{27} \mathrm{Al}$ in $\mathrm{SiC}$ grains with extremely low ${ }^{12} \mathrm{C} /{ }^{13} \mathrm{C}$ and ${ }^{14} \mathrm{~N} /{ }^{15} \mathrm{~N}$. They belong to the very small class (six known) of carbon-rich grains attributed to novae on the basis of $\mathrm{C}, \mathrm{N}$, and $\mathrm{Si}$ isotopic compositions, and they are not A and B grains (Amari et al. 2001a). No models of novae give ${ }^{14} \mathrm{~N} /{ }^{15} \mathrm{~N}$ far above unity. The yields of $\mathrm{C}, \mathrm{N}$, and $\mathrm{O}$ isotopes are all very roughly commensurate (factor of 10), so that the wide spread in ${ }^{14} \mathrm{~N} /{ }^{15} \mathrm{~N}$ cannot be achieved with low ${ }^{12} \mathrm{C} /{ }^{13} \mathrm{C}$. Thus, the high ${ }^{14} \mathrm{~N} /{ }^{15} \mathrm{~N}$ values found for some A and B grains cannot be attributed to novae. The A and B grains appear to suggest some connection with novae; however, this cannot be due to the TNRs on $\mathrm{ONeMg}$ white dwarf models for which nucleosynthesis is usually computed. One possibility for a direct nova connection would be if the fragile ${ }^{15} \mathrm{~N}$ could be destroyed during the later stages of the explosion. The models of novae so far considered have mostly high accretion rates, and it is conceivable that low accretion rates that do not initiate full TNR could produce partially processed material with isotopic characteristics resembling A and B grains. The true novae represent extremely energetic bursts punctuating a slower, ongoing accretion process. If we assume that the $\mathrm{A}$ and $\mathrm{B}$ grains were produced by a mixture of accreting AGB matter that was not processed in TNR and a component that was produced through TNR, then we immediately face the problem that the nova yield ratio $Y_{13}^{N} / Y_{15}^{N}$ is typically $\sim 0.1$, while the AGB matter with which it is mixed has almost no ${ }^{15} \mathrm{~N}$. A mixing trajectory between AGB compositions and nova compositions would therefore move rapidly from the AGB values to those with very low ${ }^{14} \mathrm{~N} /{ }^{15} \mathrm{~N}$ but high ${ }^{12} \mathrm{C} /{ }^{13} \mathrm{C}$ (see Fig. 17b). Such a mixing curve would not pass through the $\mathrm{A}$ and $\mathrm{B}$ grains. Region $\mathrm{P}$ of Figures $17 a$ and $17 b$ contains AGB compositions with extensive CBP that could mix with a nova composition to produce a mixing curve passing through the A and B grains, but only for a small corner of the parameter space where CBP produces ${ }^{12} \mathrm{C} /{ }^{13} \mathrm{C} \sim 10$ and ${ }^{14} \mathrm{~N} /{ }^{15} \mathrm{~N} \sim 2 \times 10^{4}$. Since this composition corresponds to low $T_{P}$, any ${ }^{26} \mathrm{Al}$ found in the grains would have to come from the novae. In summary, we have 
no self-consistent mechanism to propose for A and B grains. They appear to reflect different mechanisms than are currently available in stellar models. If we assume the classification of A and B grains into a single group to be invalid and consider instead subsets of the A and B grains, the problem is not removed.

\subsection{Astronomical Implications}

We now consider what evidence concerning $\mathrm{CBP}$ on the AGB should be accessible to astronomical observations. As in the case of the presolar grains, the potential for converting large amounts of $\mathrm{C}$ to $\mathrm{N}$ is crucial, and we divide up the results into those expected for AGB stars with $\mathrm{C} / \mathrm{O}>1$ and with $\mathrm{C} / \mathrm{O}<1$. This discussion applies to stars that have been on the AGB long enough to have become $\mathrm{C}$ stars without CBP; for the SEM(1.5) model, this corresponds to the star exceeding a luminosity of $1.2 \times 10^{4} L_{\odot}$.

Figure 10 shows that for almost any value of $\log T_{P}>7.60, \dot{M} \gtrsim 10^{-6} M_{\odot} \mathrm{yr}^{-1}$ can prevent carbon star formation. Low values of $\dot{M}$ are therefore required if CBP occurs in C stars. Low $\dot{M}$ requires oxygen isotopes with little or no change from the values set by first dredge-up; this means ${ }^{18} \mathrm{O} /{ }^{16} \mathrm{O} \sim 0.0015$ and wide variation of ${ }^{17} \mathrm{O} /{ }^{16} \mathrm{O}$ with stellar mass (along the thick dashed curve of Fig. 7). It may further be expected that $10^{-4}<{ }^{15} \mathrm{~N} /{ }^{14} \mathrm{~N}<6 \times 10^{-4}$ and that incomplete conversion of $\mathrm{C}$ to $\mathrm{N}$ in the envelope produces $0.5<\mathrm{N} / \mathrm{O}<0.8$ (see Figs. $12 a$ and $12 b$ ), as well as ${ }^{12} \mathrm{C} /{ }^{13} \mathrm{C}>20$. In principle, it is possible to obtain a carbon star with ${ }^{12} \mathrm{C} /{ }^{13} \mathrm{C}<20$ if $\log T_{P} \lesssim 7.6$ and $\dot{M}$ is high, but this case constitutes a very small part of the parameter space. Any level of ${ }^{26} \mathrm{Al}$ enrichment is compatible with a C-rich star, depending on $T_{P}$. We thus find that contrary to usual expectations (e.g., Arnould \& Prantzos 1999; Mowlavi \& Meynet 2000), normal low-mass $\mathrm{C}$ stars with low $\dot{M}$ could produce abundant ${ }^{26} \mathrm{Al}$, and they are reasonable targets for gamma-ray-line observations by high-energy satellite experiments like the International Gamma-Ray Astrophysical Laboratory. However, low ${ }^{12} \mathrm{C} /{ }^{13} \mathrm{C}$ requires low $T_{P}$, which is not compatible with significant ${ }^{26} \mathrm{Al} /{ }^{27} \mathrm{Al}$.

If $\mathrm{C} / \mathrm{O}<1$ in the envelope of an evolved TP-AGB star or planetary nebula, it requires $\dot{M} \gtrsim 10^{-6} M_{\odot} \mathrm{yr}^{-1}$. All of the signs of CBP that become more pronounced with greater $\dot{M}$ should then be present: ${ }^{18} \mathrm{O} /{ }^{16} \mathrm{O} \lesssim 8 \times 10^{-4}$ (Fig. 6), ${ }^{15} \mathrm{~N} /{ }^{14} \mathrm{~N} \leqslant 2 \times 10^{-4}$ (Fig. 12a), and $5<{ }^{12} \mathrm{C} /{ }^{13} \mathrm{C}<70$ (Fig. $11)$. The large quantity of carbon destroyed also requires $\mathrm{N} / \mathrm{O} \gtrsim 1.5$ (Fig. 12b). All of this processing should simultaneously destroy ${ }^{3} \mathrm{He}$. Individual objects may be found with $\mathrm{N} / \mathrm{O} \geq 3$ if $T_{P}$ is very high. Again, since ${ }^{26} \mathrm{Al}$ production is independent of $\dot{M}$, it should occur as often in O-rich as in $\mathrm{C}$-rich environments if $\mathrm{CBP}$ is active.

Large enrichments of $\mathrm{N} / \mathrm{O}$ in planetary nebulae may be important signs of CBP on the AGB. High N/O is frequently observed and is a defining characteristic of the type I planetary nebulae (see Corradi \& Schwarz 1995) that make up some $20 \%-25 \%$ of observed planetary nebulae. Past expectations have been that $\mathrm{N}$ enrichment requires $\mathrm{HBB}$ (Peimbert \& Serrano 1980; van den Hoek \& Groenewegen $1997)$ and thus intermediate-mass stars. CBP would permit $\mathrm{N}$-rich planetary nebulae to be made from low-mass $(<3$ $M_{\odot}$ ) stars. A search of planetary nebulae for other signs of CBP at high $\dot{M}$ (those associated with O-rich AGB stars), combined with good stellar mass estimates, would indicate whether the $\mathrm{N}$ originated in $\mathrm{CBP}$. Measurements of
${ }^{14} \mathrm{~N} /{ }^{15} \mathrm{~N}, \mathrm{~N} / \mathrm{O},{ }^{18} \mathrm{O} /{ }^{16} \mathrm{O},{ }^{17} \mathrm{O} /{ }^{16} \mathrm{O}$, and ${ }^{12} \mathrm{C} /{ }^{13} \mathrm{C}$ would provide clear tests of CBP. Evidence for moderate $\mathrm{N}$ enrichment in a single low-mass planetary nebula has already been found by Péquignot et al. (2000). It would be of great interest if more of these objects were found. Since the same $\mathrm{H}$-burning cycles that characterize $\mathrm{CBP}$ are also active in $\mathrm{HBB}$, it would be necessary to determine initial masses of the observed stars and nebulae in order to distinguish CBP and HBB.

\subsection{Galactic Inventories}

The extensive processing of stellar envelopes discussed above may have consequences for the overall Galactic inventories of at least two nuclides: ${ }^{3} \mathrm{He}$ and ${ }^{26} \mathrm{Al}$. Observations of ${ }^{3} \mathrm{He}$ are difficult and have so far been confined to the local ISM and some planetary nebulae (Gloeckler \& Geiss 1998; Balser et al. 1999; Bania et al. 2002). The results indicate that the Galaxy contains no more ${ }^{3} \mathrm{He}$ than one would expect from its primordial inventory plus the result of pre-main-sequence deuterium burning. It is presently not understood why so little ${ }^{3} \mathrm{He}$ has apparently been made over the lifetime of the Galaxy. Low- and intermediate-mass stars should produce large amounts of ${ }^{3} \mathrm{He}$ during their main-sequence evolution (e.g., Sackmann \& Boothroyd 1999). First dredge-up should bring much of this ${ }^{3} \mathrm{He}$ into the stellar envelopes, where subsequent mass loss allows its escape into the ISM. Conventional stellar evolution provides no way to destroy this dredged-up ${ }^{3} \mathrm{He}$. It has been noted in earlier work (WBS; Charbonnel 1995) that CBP on the RGB could provide a path to destroy ${ }^{3} \mathrm{He}$, using the reactions ${ }^{3} \mathrm{He}\left({ }^{3} \mathrm{He}, 2 p\right){ }^{4} \mathrm{He}$ and ${ }^{3} \mathrm{He}(\alpha, \gamma)^{7} \mathrm{Be}$. When we include these reactions in our nuclear reaction network, we find that extensive ${ }^{3} \mathrm{He}$ destruction also occurs in the AGB cool bottom processing scenario presented here. The results of CBP for ${ }^{3} \mathrm{He}$ are directly analogous to those for ${ }^{18} \mathrm{O}$ : there is a threshold temperature above which destruction can become rapid. We find a threshold temperature for thorough ${ }^{3} \mathrm{He}$ destruction of $\log T_{P} \sim 7.65$; a factor of 10 depletion may be reached for $\log T_{P}=7.60$. Just as for ${ }^{18} \mathrm{O}$, the final abundance of ${ }^{3} \mathrm{He}$ in the stellar envelope then depends on the rate and duration of mixing, with duration limited by the stellar lifetime. For both ${ }^{3} \mathrm{He}$ and ${ }^{18} \mathrm{O}$, essentially complete (more than a factor of 10) destruction occurs at $\dot{M} \gtrsim 3 \times 10^{-6} M_{\odot} \mathrm{yr}^{-1}$ as long as the threshold temperature is exceeded. As a result, low-mass stars that undergo $\mathrm{CBP}$ on the AGB may not be net producers of ${ }^{3} \mathrm{He}$ over their lifetimes, and this may help explain the apparently small amount of ${ }^{3} \mathrm{He}$ production that has occurred.

An observational test of whether ${ }^{3} \mathrm{He}$ is destroyed in this way may be obtained by spectroscopic measurements of ${ }^{18} \mathrm{O} /{ }^{16} \mathrm{O}$ in $\mathrm{CO}$ lines from AGB stars or planetary nebulae, since both ${ }^{18} \mathrm{O}$ and ${ }^{3} \mathrm{He}$ destruction should occur simultaneously. The threshold $T_{P}$ for ${ }^{18} \mathrm{O}$ destruction is lower than that for ${ }^{3} \mathrm{He}$ destruction, so ${ }^{18} \mathrm{O}$ destruction is required if ${ }^{3} \mathrm{He}$ is destroyed. The connection between ${ }^{3} \mathrm{He}$ and ${ }^{18} \mathrm{O}$ destruction is also important for another reason: ${ }^{3} \mathrm{He}$ destruction by CBP must occur in essentially all lowmass stars if $\mathrm{CBP}$ is to account for the lack of evolution of the ${ }^{3} \mathrm{He}$ inventory. This implies that essentially all lowmass stars should destroy most of the ${ }^{18} \mathrm{O}$ in their envelopes and, in turn, that the preponderance of oxide grains originating in these stars should have ${ }^{18} \mathrm{O} /{ }^{16} \mathrm{O}<10^{-4}$. 
Examination of Figure 16 shows that a large fraction of the sources sampled by the oxide grains have higher values, suggesting that there might not be sufficient CBP to destroy the extra ${ }^{3} \mathrm{He}$ produced in low-mass stars. This problem requires further attention.

The Galactic inventory of ${ }^{26} \mathrm{Al}$ is also of interest. The Galaxy contains $\sim 3 M_{\odot}$ of ${ }^{26} \mathrm{Al}$, as inferred from observations of the gamma-ray line from its decay (Mahoney et al. 1984; Knödlseder et al. 1996). Since ${ }^{26} \mathrm{Al}$ has a mean lifetime of $1 \mathrm{Myr}$, this requires ongoing nucleosynthesis. The sites of this nucleosynthesis remain uncertain (e.g., Knödlseder et al. 1999). If the sources of the ${ }^{26} \mathrm{Al}$ are AGB stars (which neither create nor destroy ${ }^{27} \mathrm{Al}$ ) and if the rate of injection of ${ }^{26} \mathrm{Al}$ into the ISM matches the rate of decay, then the material being ejected into the ISM must have ${ }^{26} \mathrm{Al} /{ }^{27} \mathrm{Al}$ satisfying

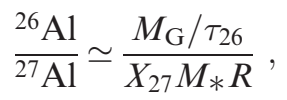

where $M_{\mathrm{G}} \sim 3 M_{\odot}$ is the Galactic ${ }^{26} \mathrm{Al}$ inventory, $X$ 's are mass fractions, $M_{*}$ is the mass of ejecta from a single AGB star, and $R$ is the death rate (stars per year) of AGB stars. If $X_{27}$ is solar, then

$$
\frac{{ }^{26} \mathrm{Al}}{{ }^{27} \mathrm{Al}}=\frac{5.4 \times 10^{-2} M_{\odot} \mathrm{yr}^{-1}}{M_{*} R}
$$

in the ejecta (see Dearborn, Lee, \& Wasserburg 1988). It is quite difficult to estimate $M_{*} R$. Consider two cases, with stellar death rates based first on the local density of giant stars and then on an estimate of the local white dwarf birth rate. In either case, we must extrapolate local stellar populations into totals for the Galaxy; since the ${ }^{26} \mathrm{Al}$ gamma-ray-line emission seems to be concentrated in the Galactic disk, we assume that the Galaxy consists only of the thin-disk component described in Binney \& Merrifield (1998), and we assume that the dying stars (i.e., those shedding their envelopes into the ISM and evolving into white dwarfs) make up the same fraction of stars everywhere in the Galaxy as they do locally. Using the local density of red giant stars from Binney \& Merrifield (1998) and assuming that each of these stars sheds its envelope after a lifetime of $10^{8} \mathrm{yr}$, we find $R=1.3$ $\mathrm{yr}^{-1}$. If these stars each eject $M_{*} \sim 1 M_{\odot}$, then the ejecta must have ${ }^{26} \mathrm{Al} /{ }^{27} \mathrm{Al}=4 \times 10^{-2}$, from equation (28). Figure $3 a$ shows that this level could be produced by CBP with $\log T_{P} \sim 7.75$. Extensive CBP at high $T_{P}$ might permit AGB stars to be a significant source of the Galactic ${ }^{26} \mathrm{Al}$. However, the preponderance of circumstellar grains appear to be produced in environments where ${ }^{26} \mathrm{Al} /{ }^{27} \mathrm{Al}$ is much below $4 \times 10^{-2}$. If we use the white dwarf birth rate of Drilling \& Schönberner (1985) and we extrapolate the local rate of $(2-3) \times 10^{-13} \mathrm{pc}^{-1} \mathrm{yr}^{-1}$ to a total Galactic rate, we obtain $R=0.04-0.06 \mathrm{yr}^{-1}$. This is far too low to contribute to the Galactic inventory. We conclude that it is unlikely that a significant fraction of the ${ }^{26} \mathrm{Al}$ observed in gamma-ray-line emission in the Galaxy was made in AGB stars. This conclusion has also been reached on the basis of the apparently clumpy distribution of ${ }^{26} \mathrm{Al}$ in the Galaxy (Diehl \& Timmes 1998). However, $\mathrm{CBP}$ in $\mathrm{AGB}$ stars may provide sufficient ${ }^{26} \mathrm{Al}$ to permit detection in selected AGB stars with very low ${ }^{18} \mathrm{O} /{ }^{16} \mathrm{O}$ and high $\mathrm{N} / \mathrm{O}$.

\section{CONCLUSION}

The work presented in this paper demonstrates that extensive predictions of the possible range of envelope compositions and isotopic abundances of AGB stars can be made in a model of cool bottom processing parameterized only by a mass-flow rate $\dot{M}$ and a maximum temperature $T_{P}$ using a simple calculation.

This calculation uses results from a stellar evolution model calculated without $\mathrm{CBP}$ to provide the basic conditions needed to determine the results of additional processing by extra mixing. It appears that significant composition changes have negligible feedback on the stellar luminosity. As a result, the postprocessing approach adopted here - in which CBP is calculated separately from stellar evolution - appears to be reasonable. Postprocessing calculations allow the range of possible effects of extra mixing to be determined easily by exploring the space of $T_{P}$ and $\dot{M}$ values once stellar structures have been determined for an arbitrary star. The results reported in this work are based on a grid of 231 pairs of the two parameters. This covers the entire range that is both plausible and potentially interesting, using an unsophisticated code and about 1 day of processing time on a personal computer with $930 \mathrm{MHz}$ clock speed. Moreover, given only the stellar structure and reaction rates, one can obtain almost quantitatively correct analytical approximations to these results as functions of $\dot{M}$ and $T_{P}$ for C/O (eq. [22] and Fig. 10), ${ }^{26} \mathrm{Al} /{ }^{27} \mathrm{Al}$ (eq. [6] and Fig. $3 a$ ), ${ }^{18} \mathrm{O} /{ }^{16} \mathrm{O}$ (eq. [13] and Fig. 4), and ${ }^{17} \mathrm{O} /{ }^{16} \mathrm{O}$ (eq. [16] and Fig. 5). Given the uncertainties concerning the exact mass, evolutionary state, mass-loss rate, and extra-mixing parameters corresponding to the star that produced an observed presolar grain or planetary nebula, the analytic approximations are reasonably accurate and display in a clear way the rules that govern the outcome of CBP.

We also found that given the uncertainties (particularly the unknown mixing mechanism, which sets the mixing depth), the detailed evolution of the stellar structure below the stellar envelope (e.g., the radiative zone) is relatively unimportant. The results from a complete stellar evolution code without extra mixing are necessary to specify the timescales, the evolution of envelope mass, the action of dredgeup, and a representative structure for the region in which nuclear processing takes place in the CBP model. However, the detailed evolution of this region need not be followed to obtain useful results. The major effect of the evolution of this region is to change the $T_{P}$ that corresponds to a given amount of processing; holding both the structure of this region and $T_{P}$ fixed is equivalent to holding $T_{P} / T_{\mathrm{H}}$ fixed in a model that follows the detailed evolution, provided that both follow evolution of the envelope in the same way.

The effects of CBP on stars of different masses or metallicities (particularly low metallicity) can readily be determined once a conventional non-CBP stellar model and radiativezone structure are obtained from conventional codes.

We gratefully acknowledge the use of the FRANEC code, granted by O. Straniero, A. Chieffi, and M. Limongi, as well as extensive comments and suggestions from J. Lattanzio and an anonymous referee. Some incisive comments by R. Gallino were valuable and helpful. M. B. wishes to thank The Lunatic Asylum and Caltech for hospitality. This work was supported by NASA grant NAG5-11725 and by Italian MURST contract Cofin2000. This work is Caltech division contribution 8769(1080). 


\section{APPENDIX}

\section{REACTION RATE UNCERTAINTIES}

Most of the reaction rates used in this paper are known to within 5\%-40\%, according to the NACRE evaluation (Angulo et al. 1999). There are a few cases of larger uncertainty, which we now examine. In cases where more or less reliable error estimates exist, the NACRE upper and lower limits to the reaction rates are often found by multiplying by 2 the size of the statistical error bars that one derives directly from the experimental reports. We consider the rates at a temperature of $5 \times 10^{7} \mathrm{~K}$ as representative of the crucial temperature range.

First, the NACRE compilation quotes uncertainties of a factor of 2 for the rate of ${ }^{18} \mathrm{O}(p, \alpha){ }^{15} \mathrm{~N}$. This affects only the rate of ${ }^{18} \mathrm{O}$ destruction at low $T_{P}\left(\lesssim 10^{7.62} \mathrm{~K}\right)$. Above this temperature, increasing the destruction rate changes nothing because even with the recommended rates, a single pass through the radiative zone suffices to greatly reduce the ${ }^{18} \mathrm{O}$ abundance in the circulating matter. Likewise, decreasing this rate will have no effect as long as the lower rate still suffices to destroy all ${ }^{18} \mathrm{O}$ in a single pass; the threshold $T_{P}$ for this condition is also about $10^{7.62} \mathrm{~K}$. Below $\log T_{P}=7.62$, factor of 2 changes in the ${ }^{18} \mathrm{O}$ destruction rate translate into factor of 2 changes in the final ${ }^{18} \mathrm{O} /{ }^{16} \mathrm{O}$.

The most important uncertainties are those concerning reactions that produce and destroy ${ }^{26} \mathrm{Al}$. The NACRE compilation quotes upper and lower limits for the rate of ${ }^{26} \mathrm{Al}^{9}(p, \gamma)^{27} \mathrm{Si}$ that are a factor of 47 higher and a factor of 86 lower, respectively, than the recommended rate. It should be kept in mind that the NACRE recommended rate at these temperatures was determined mainly by setting resonance strengths to be $1 / 10$ of the upper limits derived from the shell-model calculation of Champagne, Brown, \& Sherr (1993; these are considerably lower than the experimental upper limits). Changing from the NACRE recommended rate to the NACRE upper limit rate reduces the equilibrium ${ }^{26} \mathrm{Al} /{ }^{25} \mathrm{Mg}$ in circulating material from near 100 to around unity. As a result, low $\dot{M}$ then allows this ratio to equilibrate in the circulating material, so that the proportionality of ${ }^{26} \mathrm{Al}$ production to $\dot{M}^{-1}$ in equation (4) breaks down, and the amount of ${ }^{26} \mathrm{Al}$ returning to the surface becomes both lower and independent of $\dot{M}$. Although the final ${ }^{26} \mathrm{Al} /{ }^{27} \mathrm{Al}$ in the envelope is smaller in this case, it still remains high enough to account for all oxide grains with only a very slight increase in the maximum $T_{P}$.

The rate of the ${ }^{26} \mathrm{Al}$ production reaction, ${ }^{25} \mathrm{Mg}(p, \gamma)^{26} \mathrm{Al}^{g}$, is quoted with an upper limit $75 \%$ above the recommended rate and a lower limit $41 \%$ below the recommended rate. The NACRE rate and uncertainties for this reaction are based on resonance strengths extracted by Iliadis et al. (1996) from preexisting data using improved distorted-wave Born approximation methods. Except at $\dot{M} \lesssim 10^{-7} M_{\odot} \mathrm{yr}^{-1}$, where the independence of the envelope ${ }^{26} \mathrm{Al}$ enrichment rate from $\dot{M}$ breaks down, the result of varying the ${ }^{25} \mathrm{Mg}(p, \gamma){ }^{26} \mathrm{Al}$ rate within the NACRE limits is to scale the final ${ }^{26} \mathrm{Al} /{ }^{27} \mathrm{Al}$ downward by as much as $40 \%$ or upward by up to $75 \%$.

The only other large uncertainties (greater than a factor of 2) in the reaction rates included in our code are for the ${ }^{27} \mathrm{Al}-$ producing and -destroying reactions ${ }^{26} \mathrm{Mg}(p, \gamma){ }^{27} \mathrm{Al},{ }^{27} \mathrm{Al}(p, \alpha){ }^{24} \mathrm{Mg}$, and ${ }^{27} \mathrm{Al}(p, \gamma){ }^{28} \mathrm{Si}$. The first of these reactions has an upper limit in the NACRE compilation that is 15 times the recommended rate (derived mainly from experimental work by Champagne et al. 1990) and a lower limit that is $1 / 27$ the recommended rate. The upper and lower limits to the $(p, \alpha)$ destruction rate differ from the recommended rate by factors of 10 and 5, respectively. To examine the allowed effect on ${ }^{26} \mathrm{Al} /{ }^{27} \mathrm{Al} \mathrm{due}$ to ${ }^{27} \mathrm{Al}$ production and destruction (which is negligible when using the recommended rates), we ran models with all of the ${ }^{27} \mathrm{Al}-$ producing reaction rates [including the ${ }^{26} \mathrm{Al}$-destroying ${ }^{26} \mathrm{Al}(p, \gamma)^{27} \mathrm{Si}\left(\beta^{+} \nu\right)^{27} \mathrm{Al}$ ] set to the NACRE upper limits and the ${ }^{27} \mathrm{Al}-$ destroying reaction rates set to their NACRE lower limits. This made virtually no difference to the final ${ }^{26} \mathrm{Al} /{ }^{27} \mathrm{Al}$ beyond the effects of ${ }^{26} \mathrm{Al}(p, \gamma){ }^{27} \mathrm{Si}$ on ${ }^{26} \mathrm{Al}$ production described above. The corresponding calculation with the limits switched to minimize the final ${ }^{27} \mathrm{Al}$ abundance showed no significant difference from that using the recommended rates.

\section{REFERENCES}

Alexander, C. M. O., \& Nittler, L. R. 1999, ApJ, 519, 222

Amari, S., Gao, X., Nittler, L. R., Zinner, E., José, J., Hernanz, M., \& Lewis, R. S. 2001a, ApJ, 551, 1065

Amari, S., Lewis, R. S., \& Anders, E. 1994, Geochim. Cosmochim. Acta, 58,459

Amari, S., Nittler, L. R., Zinner, E., Gallino, R., Lugaro, M., \& Lewis, R. S. 2001 b, ApJ, 546, 248

Amari, S., Nittler, L. R., Zinner, E., Lodders, K., \& Lewis, R. S. 2001c, ApJ, 559, 463

Angulo, C., et al. 1999, Nucl. Phys. A, 656, 3

Arnould, M., \& Prantzos, N. 1999, NewA, 4, 283

Balser, D. S., Bania, T. M., Rood, R. T., \& Wilson, T. L. 1999, ApJ, 510, 759

Bania, T. M., Rood, R. T., \& Balser, D. S. 2002, Nature, 415, 54

Binney, J., \& Merrifield, M. 1998, Galactic Astronomy (Princeton: Princeton Univ. Press)

Blackmon, J. C., Champagne, A. E., Hofstee, M. A., Smith, M. S., Downing, R. G., \& Lamaze, G. P. 1995, Phys. Rev. Lett., 74, 2642

Boothroyd, A. I., \& Sackmann, I.-J. 1999, ApJ, 510, 232

Boothroyd, A. I., Sackmann, I.-J., \& Wasserburg, G. J. 1994, ApJ, 430, L77

. 1995, ApJ, 442, L21

Busso, M., Gallino, R., Lambert, D. L., Travaglio, C., \& Smith, V. V. 2001, ApJ, 557, 802

Busso, M., Gallino, R., \& Wasserburg, G. J. 1999, ARA\&A, 37, 239 (BGW99)

Cavallo, R., Sweigart, A., \& Bell, R. 1998, ApJ, 492, 575

Champagne, A. E., Brown, B. A., \& Sherr, R. 1993, Nucl. Phys. A, 556, 123
Champagne, A. E., Magnus, P. V., Smith, M. S., \& Howard, A. J. 1990, Nucl. Phys. A, 512, 317

Champagne, A. E. \& Pitt, M. L. 1986, Nucl. Phys. A, 457, 367

Charbonnel, C. 1995, ApJ, 453, L41

Charbonnel, C., Brown, J. A., \& Wallerstein, G. 1998, A\&A, 332, 204

Chin, Y.-N., Henkel, C., Langer, N., \& Mauersberger, R. 1999, ApJ, 512, L143

Choi, B.-G., Huss, G. R., Wasserburg, G. J., \& Gallino, R. 1998, Science, 282,1284

Choi, B.-G., Wasserburg, G. J., \& Huss, G. R. 1999, ApJ, 522, L133

Corradi, R. L. M., \& Schwarz, H. E. 1995, A\&A, 293, 871

Da Costa, G. S. 1998, in IAU Symp. 189, Fundamental Stellar Properties: The Interaction between Observation and Theory, ed. T. R. Bedding, A. J. Booth, \& J. Bavis (Dordrecht: Kluwer), 193

Dearborn, D. S. P. 1992, Phys. Rep., 210, 367

Dearborn, D. S. P., Lee, T., \& Wasserburg, G. J. 1988, Lunar Planet. Sci. Conf., 19, 257

Denissenkov, P. A., Da Costa, G. S., Norris, J. E., \& Weiss, A. 1998, A\&A, 333,926

Denissenkov, P. A., \& Weiss, A. 1996, A\&A, 308, 773

. 2001, ApJ, 559, L115

Diehl, R., \& Timmes, F. 1998, PASP, 110, 637

Drilling, J. S., \& Schönberner, D. 1985, A\&A, 146, L23

El Eid, M. 1994, A\&A, 285, 915

Forestini, M., Arnould, M., \& Paulus, G. 1991, A\&A, 252, 597

Frost, C. A., Cannon, R. C., Lattanzio, J. C., Wood, P. R., \& Forestini, M. 1998, A\&A, 332, L17

Gallino, R., Arlandini, C., Busso, M., Lugaro, M., Travaglio, C., Straniero, O., Chieffi, A., \& Limongi, M. 1998, ApJ, 497, 388 
Gallino, R., Busso, M., \& Lugaro, M. 1997, in AIP Conf. Proc. 402, Astrophysical Implications of the Laboratory Study of Presolar Materials, ed. T. J. Bernatowicz \& E. Zinner (New York: AIP), 115

Gallino, R., Raiteri, C. M., Busso, M., \& Matteucci, F. 1994, ApJ, 430, 858

Gehrz, R. D., Truran, J. W., Williams, R. E., \& Starrfield, S. 1998, PASP, 110,3

Gilroy, K. K., \& Brown, J. A. 1991, ApJ, 371, 578

Gloeckler, J., \& Geiss, G. 1998, Space Sci. Rev., 84, 239

Gratton, R., Sneden, C., Caretta, E., \& Bragaglia, A. 2000, A\&A, 354, 169

Harris, M. J., Lambert, D. L., Hinkle, K. H., Gustafsson, B., \& Eriksson, K. 1987, ApJ, 316, 294

Harris, M. J., Lambert, D. L., \& Smith, V. V. 1985, ApJ, 299, 375

Herwig, F., Blöcker, T., Schönberner, D., \& El Eid, M. 1997, A\&A, 324, L81

Hoppe, P., Amari, S., Zinner, E., Ireland, T., \& Lewis, R. S. 1994, ApJ, 430, 870

Hoppe, P., \& Ott, U. 1997, in AIP Conf. Proc. 402, Astrophysical Implications of the Laboratory Study of Presolar Materials, ed. T. J. Bernatowicz \& E. Zinner (New York: AIP), 27

Huss, G. R., Fahey, A. J., Gallino, R., \& Wasserburg, G. J. 1994, ApJ, 430, L81

Huss, G. R., Hutcheon, I. D., \& Wasserburg, G. J. 1997, Geochim. Cosmochim. Acta, 61, 5117

Hutcheon, I. D., Huss, G. R., Fahey, A., \& Wasserburg, G. J. 1994, ApJ, 425, L97

Iben, I., Jr., \& Renzini, A. 1983, ARA\&A, 21, 271

Iliadis, C., Buchmann, L., Endt, P. M., Herndl, H., \& Wiescher, M. 1996, Phys. Rev. C, 53, 475

Kahane, C., Cernicharo, J., Gómez-González, J., \& Guélin, M. 1992, A\&A, 256, 235

Knödlseder, J., et al. 1996, A\&AS, 120, 335 1999, A\&A, 344, 68

Kraft, R. P. 1994, PASP, 106, 553

Langer, G. E., Hoffman, R. D., Sneden, C. 1993, PASP, 105, 301

Lattanzio, J., Forestini, M., \& Charbonnel, C. 2000, Mem. Soc. Astron. Italiana, 71, 737

Lattanzio, J. C., \& Forestini, M. 1999, in IAU Symp. 191, Asymptotic Giant Branch Stars, ed. T. Le Bertre, A. Lèbre, \& C. Waelkens (San Francisco: ASP), 447

Lee, T., Papanastassiou, D., \& Wasserburg, G. J. 1977, ApJ, 211, L107

Maeder, A., \& Zahn, J. P. 1998, A\&A, 334, 1000
Mahoney, W. A., Ling, J. C., Wheaton, W. A., \& Jacobson, A. S. 1984, ApJ, 286, 578

Messenger, B. 2000, Ph.D. thesis, Monash Univ.

Mowlavi, N., \& Meynet, G. 2000, A\&A, 361, 959

Nicolussi, G. K., Davis, A. M., Pellin, M. J., Lewis, R. S., Clayton, R. N., \& Amari, S. 1997, Science, 277, 1281

Nicolussi, G. K., Pellin, M. F., Lewis, R. S., Davis, A. M., Amari, S., \& Clayton, R. N. 1998, Geochim. Cosmochim. Acta, 62, 1093

Nittler, L. R., Alexander, C. M. O'D., Gao, X., Walker, R. M., \& Zinner, E. 1994, Nature, 370, 443

Nittler, L. R., Alexander, C. M. O’D., Wang, J., \& Gao, X. 1998, Nature, 393,222

Nittler, L. R., \& Cowsik, R. 1996, Meteoritics Planet. Sci., 31, A100 1997, Phys. Rev. Lett. 78,175

Peimbert, M., \& Serrano, A. 1980, Rev. Mexicana Astron. Astrofis., 5, 9

Pellin, M. J., Calaway, W. F., Davis, A. M., Lewis, R. S., Amari, S., \& Clayton, R. N. 2000, Lunar Planet. Sci. Conf., 31, 1917

Penzias, A. A. 1981, ApJ, 249, 518

Péquignot, D., Walsh, J. R., Zijltra, A. A., \& Didziak, G. 2000, A\&A, 361, L1

Reimers, D. 1975, in Problems in Stellar Atmospheres and Envelopes, ed. A. Unsöold, B. Baschek, W. H. Kegel, \& G. Traving (Berlin: Springer), 229

Sackmann, I.-J., \& Boothroyd, A. I. 1999, ApJ, 510, 217

Starrfield, S., Iliadis, C., Truran, J. W., Wiescher, M., \& Sparks, W. M. 2001, Nucl. Phys. A, 688, 110c

Straniero, O., Chieffi, A., Limongi, M., Busso, M., Gallino, R., \& Arlandini, C. 1997, ApJ, 478, 332

Sweigart, A. V. 1997, ApJ, 474, L23

Sweigart, A. V., \& Mengel, J. G. 1979, ApJ, 229, 624

Timmes, F. X., \& Clayton, D. D. 1996, ApJ, 472, 723

van den Hoek, L. B., \& Groenewegen, M. A. T. 1997, A\&AS, 123, 305

Wasserburg, G. J., Boothroyd, A. I., \& Sackmann, I.-J. 1995, ApJ, 447, L37 (WBS)

Wasserburg, G. J., Busso, M., Gallino, R., \& Raiteri, C. M. 1994, ApJ, 424, 412

Wasserburg, G. J., Gallino, R., Busso, M., Goswami, J. N., \& Raiteri, C. M. 1995, ApJ, 440, L101

Weiss, A., Denissenkov, P. A., \& Charbonnel, C. 2000, A\&A, 356, 181

Wilson, T. L., \& Rood, R. T. 1994, ARA\&A, 32, 191

Zahn, J. P. 1992, A\&A, 265, 115 